\title{
SPECTROSCOPIC BINARIES, VELOCITY JITTER, AND ROTATION IN FIELD METAL-POOR RED GIANT AND RED HORIZONTAL-BRANCH STARS
}

\author{
BRUCE W. CARNeY \\ Department of Physics and Astronomy, University of North Carolina, Chapel Hill, NC 27599-3255; bruce@physics.unc.edu \\ David W. Latham and Robert P. Stefanik \\ Harvard-Smithsonian Center for Astrophysics,60 Garden Street, Cambridge, MA 02138; dlatham@cfa.harvard.edu, rstefanik@cfa.harvard.edu \\ JOHN B. LAIRD \\ Department of Physics and Astronomy, Bowling Green State University, Bowling Green, OH 43403; laird@tycho.bgsu.edu \\ AND \\ JON A. MORSE \\ Center for Astrophysics and Space Astronomy, Department of Astrophysical and Planetary Sciences, \\ University of Colorado, Boulder, CO 80309-0389; morsey@casa.colorado.edu \\ Received 2002 August 19; accepted 2002 October 2
}

\begin{abstract}
We summarize 2007 radial velocity measurements of 91 metal-poor field red giants. Excluding binary systems with orbital solutions, our coverage averages $13.7 \mathrm{yr}$ per star, with a maximum of $18.0 \mathrm{yr}$. We report four significant findings. (1) Sixteen stars are found to be spectroscopic binaries, and we present orbital solutions for 14 of them. The spectroscopic binary frequency of the metal-poor red giants, with $[\mathrm{Fe} / \mathrm{H}] \leq-1.4$, for periods less than 6000 days, is $16 \% \pm 4 \%$, which is not significantly different from that of comparable-metallicity field dwarfs, $17 \% \pm 2 \%$. The two $\mathrm{CH}$ stars in our program, $\mathrm{BD}-1^{\circ} 2582$ and $\mathrm{HD}$ 135148 , are both spectroscopic binaries. (2) Velocity jitter is present among about $40 \%$ of the giants with $M_{V} \leq-1.4$. The two best-observed cases, HD 3008 and BD $+22^{\circ} 2411$, show pseudoperiodicities of 172 and 186 days, longer than any known long-period variable in metal-poor globular clusters. Photometric variability seen in HD 3008 and three other stars showing velocity jitter hints that starspots are the cause. However, the phasing of the velocity data with the photometry data from Hipparcos is not consistent with a simple starspot model for HD 3008. We argue against orbital motion effects and radial pulsation, so rotational modulation remains the best explanation. The implied rotational velocities for HD 3008 and BD $+22^{\circ} 2411$, both with $M_{V} \leq-1.4$ and $R \approx 50 R_{\odot}$, exceed $12 \mathrm{~km} \mathrm{~s}^{-1}$. (3) Including HD 3008 and $\mathrm{BD}+22^{\circ} 2411$, we have found signs of significant excess line broadening in eight of the 17 red giants with $M_{V} \leq-1.4$, which we interpret as rotation. In three cases, $\mathrm{BD}+30^{\circ} 2034, \mathrm{CD}-37^{\circ} 14010$, and $\mathrm{HD} 218732$, the rotation is probably induced by tidal locking between axial rotation and the observed orbital motion with a stellar companion. But this cannot explain the other five stars in our sample that display signs of significant rotation. This high frequency of elevated rotational velocities does not appear to be caused by stellar mass transfer or mergers: there are too few main-sequence binaries with short enough periods. We also note that the lack of any noticeable increase in mean rotation at the magnitude level of the red giant branch luminosity function "bump" argues against the rapid rotation's being caused by the transport of internal angular momentum to the surface. Capture of a planetary-mass companion as a red giant expands in radius could explain the high rotational velocities. (4) We also find significant rotation in at least six of the roughly $15(40 \%)$ red horizontal-branch stars in our survey. It is likely that the enhanced rotation seen among a significant fraction of both blue and red horizontal-branch stars arose when these stars were luminous red giants. Rapid rotation alone therefore appears insufficient cause to populate the blue side of the horizontal branch. While the largest projected rotational velocities seen among field blue and red horizontal-branch stars are consistent with their different sizes, neither are consistent with the large values we find for the largest red giants. This suggests that some form of angular momentum loss (and possibly mass loss) has been at work. Also puzzling is the apparent absence of rotation seen in field RR Lyrae variables. Angular momentum transfer and conservation in evolved metalpoor field stars thus pose many interesting questions for the evolution of low-mass stars.
\end{abstract}

Key words: binaries: spectroscopic — Galaxy: halo — planetary systems: formation stars: Population II — stars: rotation

On-line material: machine-readable table

\section{INTRODUCTION}

Halo red giants are laboratories with which to explore a number of fascinating physical processes. In this paper, we explore several key questions and offer possible solutions.

The first question has to do simply with the binary frequency among such stars in the field. This is important for several reasons. For example, a comparison of the spectroscopic binary star frequencies and orbital parameters between the metal-poor field red giants and the antecedent class of metal-poor main-sequence stars could measure the fraction of such dwarf systems that subsequently underwent stellar mergers. Only a small fraction is expected, since only a small percentage of the dwarf star spectroscopic binary 
systems have short enough periods and small enough separations for an expanding red giant photosphere to engulf them. The binary fraction of field red giants may also bear on the dynamical evolution of globular clusters. Binary evolution in the dense stellar environments found in globular clusters can have important consequences for the clusters' dynamical evolution (see the excellent review by Hut et al. 1992). Modeling these phenomena requires an understanding of the initial binary frequencies and orbital characteristics, which the field stars might illuminate. Alternatively, a comparison of the frequencies of binaries and the distributions of their orbital elements between field stars and similar stars in dynamically unevolved clusters would be of interest, assuming that the field stars formed in lower density environments than have the cluster members.

A second question involves "velocity jitter." As first noted by Gunn \& Griffin (1979), a fraction of the most luminous stars in the globular cluster M3 show velocity variability that does not appear to be due to orbital motion, and which has been termed velocity jitter. The much nearer, brighter field stars offer an opportunity to more easily study the phenomenon over a wide range of luminosities and over a long period of time.

A third question is why a significant fraction of field and cluster blue horizontal-branch stars rotate (see Peterson, Rood, \& Crocker 1995; Kinman et al. 2000). Red giants are the evolutionary state that precedes core helium burning horizontal-branch stars, and thus a study of rotation among red giants may help us understand the origin of the enhanced rotation found among blue horizontal-branch stars. Angular momentum could be transferred from rapidly spinning red giant cores, or it could have been provided from close companions. Our results are not consistent with internal angular momentum transport, but they are consistent in three cases with tidal locking in a stellar binary system. We speculate that absorption of nearby planets could explain the remaining cases of enhanced rotation we find among the most luminous red giants. Stellar tidal interactions or planetary absorption may explain the rotation that has been found among blue horizontal-branch (BHB) stars. Little attention has been paid to the rotation of red horizontal-branch (RHB) stars, and we also address that issue.

Finally, there appears to be an interesting difference between metal-poor red giants found in the field and those residing within globular clusters, which might be related to rotation. In globular clusters, clear evidence has been found for anticorrelations between abundances of sodium and oxygen, and even between magnesium and aluminum in some cases (see, e.g., Sneden et al. 1991; Kraft et al. 1992; Sneden et al. 1992; Kraft et al. 1993; Sneden et al. 1994; Kraft et al. 1995; Pilachowski et al. 1996b; Kraft et al. 1997; Sneden et al. 1997; Kraft et al. 1998; Ivans et al. 1999, 2001). These elemental abundance variations may be caused either by deep mixing or by primordial variations. What is intriguing is that the field red giants of similar metallicity do not appear to display such a large range in abundance variations (see Kraft 1994 for an excellent review, as well as extensive studies of field stars by Shetrone 1996, Pilachowski, Sneden, \& Kraft 1996a, Hanson et al. 1998, and Gratton et al. 2000). What is special about living in a globular cluster? Are the abundance variations due to the increased likelihood of "primordial" abundance variations resulting from a larger number of local supernovae or asymptotic giant branch (AGB) stars? Or are the variations in cluster stars' abundances due to some more subtle influence, such as tidal interactions that distort the distributions of stellar rotational velocities and the internal mixing induced thereby? Binary red giants with small separations may have experienced such tidal interactions and thereby provide us with intriguing targets for abundance analyses to see if they alone among the field stars display the abundance variations seen so commonly in cluster giants. A nearly circular orbit is a sign of such an interaction.

\section{SELECTION OF STARS FOR STUDY}

For intervals ranging up to 20 years, we have been monitoring the radial velocities of over 1400 stars selected from catalogs of high proper motion stars. These data have revealed a large number of spectroscopic binary stars (Latham et al. 1988, 1992, 2002; Goldberg et al. 2002; Carney et al. 2001) and are enabling us to study the spectroscopic binary frequency, orbital characteristics, and secondary mass distributions of metal-poor halo stars for comparison with their metal-rich disk population counterparts. Carney \& Latham (1986, hereafter CL86) reported 337 new radial velocities for 84 metal-poor field red giants, and employing these results and velocities found in the literature, they estimated the velocity ellipsoid of the metal-poor field red giant stars. For all the reasons cited in $\S 1$, we have continued to monitor the velocities of the stars observed for CL86.

CL86 discusses the criteria by which a list of kinematically unbiased metal-poor $([\mathrm{Fe} / \mathrm{H}] \leq-1.5)$ red giants was assembled, relying primarily on the objective-prism spectroscopy and uvby photometry of Bond (1980) and the DDO and $R I_{\mathrm{C}}$ photometry of Norris, Bessell, \& Pickles (1985, hereafter NBP). We have added seven stars to the CL86 observing program: HD 108317, 111721, 110885, and 126587, BD + $13^{\circ} 3683$, HD 171496, and HD 175305. Three of these, HD 108317, 111721, and 126587, were discussed in CL86, but no new velocities were reported. All these stars have been classified as metal-poor red giants by AnthonyTwarog \& Twarog (1994, hereafter ATT), and all have very small Hipparcos parallaxes consistent with this identification. The failure to meet this latter criterion led us to remove HD 161770 and HD 205650 from our original program. We also included HD 142691 in our program but had to remove it later. ATT pointed out that the star deviates strongly in the $c_{0}$ versus $(b-y)_{0}$ plane, and this is probably caused by its nearby (0".33) and hot companion (Parsons \& Ake 1998). A high-resolution, high signal-to-noise ratio $(\mathrm{S} / \mathrm{N})$ spectrum obtained with the Kitt Peak National Observatory echelle spectrograph and $4 \mathrm{~m}$ telescope revealed a relatively strong absorption-line spectrum (and a strong lithium line). A preliminary analysis found $[\mathrm{Fe} / \mathrm{H}] \approx-0.3$. This result, plus the star's low space velocity, suggests that it is more massive and younger than our sample of metal-poor, high-velocity red giants. In this paper, we summarize the results obtained from 2007 spectroscopic observations of the 91 stars.

\section{OBSERVATIONS}

The spectra of our program stars have been obtained using the Center for Astrophysics Digital Speedometers (Latham 1985, 1992), primarily with the $1.5 \mathrm{~m}$ Wyeth reflector at the Oak Ridge Observatory in Harvard, Massachusetts. We have also used the $1.5 \mathrm{~m}$ Tillinghast 
reflector and the Multiple Mirror Telescope atop Mount Hopkins in Arizona, especially for the stars south of $-20^{\circ}$ and north of $+62^{\circ}$ in declination. The wavelength coverage is $45 \AA$, centered near $5187 \AA$, with a resolution of $8.5 \mathrm{~km}$ $\mathrm{s}^{-1}$. The $\mathrm{S} / \mathrm{N}$ varied from 10 to 50 per resolution element, with a typical value of about 15 .

The radial velocities have been determined using crosscorrelation methods, originally using three high-S/N observed spectra as templates: those for a giant A star, the sky, and an M giant, as discussed in CL86. Here, for two reasons, we have measured radial velocities using synthetic rather than observed spectra as templates for our correlation analyses. First, it was our expectation (confirmed by experiment) that templates selected from a grid to match the estimated temperatures, metallicities, and gravities of our program stars would yield better correlations and improve our ability to detect velocity variability. Guided by model isochrones, we calculated a grid of model atmospheres in temperature-gravity-metallicity space that would span the properties of our program stars. Synthetic spectra were computed for each model in the grid, and radial velocities for each star were then determined using the most appropriate synthetic spectrum as the template. Second, synthetic spectra enabled us to include rotational broadening so that we may estimate values of $v_{\text {rot }} \sin i$ by careful comparison with the observed spectra.

The starting model atmospheres employed were those of Kurucz (1992), and a description of the templates, and the procedures used to calculate synthetic spectra, including the effects of rotation, is given by Nordström et al. (1994). All models were computed using opacity distribution functions and elemental abundances in which all the " $\alpha$ " elements $(\mathrm{O}$, $\mathrm{Ne}, \mathrm{Mg}, \mathrm{Si}, \mathrm{S}, \mathrm{Ar}, \mathrm{Ca}$, and Ti) were enhanced by 0.4 dex relative to the solar abundances. The run of Rosseland optical depths ranged from $\log \tau_{\text {Ross }}=-6.875$ to $\log \tau_{\text {Ross }}=+2.0$ in steps of 0.125 (i.e., 72 depths). All models were iterated until the flux was constant to within 1\% at all depths (and was always very much better than this at all depths above the convection zone). The program SYNTHE was then used to compute synthetic spectra in the wavelength range 5146 to $5229 \AA$. The "line list" was developed by modifying theoretical $g f$-values and central wavelengths for atomic and molecular absorption lines so that an excellent line-by-line match were achieved when the Kurucz solar model and synthetic spectrum were compared with the observed solar flux spectrum (Kurucz et al. 1984). SYNTHE computed the specific intensity at 17 different emergent angles across the stellar disk. Integration over the disk, including the effects of stellar rotation, yielded the synthetic flux spectrum. The grid spacing was $250 \mathrm{~K}$ in temperature, $0.5 \mathrm{dex}$ in $\log g$, and 0.5 dex in metallicity. At each combination of temperature, gravity, and metallicity, in our grid we computed synthetic spectra employing a wide range of rotational broadening profiles, with $v_{\text {rot }}$ of $0,1,2,4,6,8,10,12,16,20,25,30,35$, $40,50,60,70,80,90,100,110,120$, and $140 \mathrm{~km} \mathrm{~s}^{-1}$. SYNTHE also allows for instrumental broadening, which we chose to be a Gaussian with FWHM of $8.5 \mathrm{~km} \mathrm{~s}^{-1}$, which is appropriate to the instrumentation we employed. The adopted turbulent velocities were $2 \mathrm{~km} \mathrm{~s}^{-1}$.

\section{STELLAR PARAMETERS}

We are interested in the estimation of stellar atmospheric parameters to select the optimal template for comparison with the observed spectra for each star. From these parameters, we also derive an estimate of each star's radius, which, for three reasons, is of fundamental importance. First, tidal interactions and mass transfer between components in a binary system depend on the ratio of the separation (estimated from the orbital period) and the stellar radii. At a fixed separation, stars with larger radii are more likely to have had their orbital and rotational properties altered by such interactions. Orbital periods and eccentricities are more readily altered when giant stars are involved, and mergers also become more likely. Thus, any comparison of spectroscopic binary frequencies or orbital properties must take the relative stellar radii into account. Second, velocity jitter among halo red giants appears to be correlated with evolutionary state, manifested by stellar luminosity or temperature, appearing predominantly at the higher luminosities and cooler temperatures. As we describe below, it appears that stellar rotation arises unexpectedly among some stars of advanced evolutionary state, including the largest, coolest, most luminous red giants and their descendents, horizontal-branch stars. Discriminating between lower and upper giant branch stars, as well as horizontal-branch stars, is crucial to our analyses. Third, there may be cases in which a rotational timescale may be determined, and a stellar radius may then be used to derive a rotational velocity independent of the inclination of the rotational axis to our line of sight.

\subsection{Reddening, Absolute Magnitude, and $[\mathrm{Fe} / \mathrm{H}]$}

Estimation of reddening, temperature, gravity, and metallicity for evolved field stars can be a difficult process. ATT extended the earlier work of Bond (1980) by increasing considerably the number of stars with uvby photometry and by use of the Burstein \& Heiles (1982) reddening maps rather than a simple cosecant law. Wherever possible, we have adopted their results or, in the cases where new uvby photometry is available, their procedure. In particular, we have employed the updated photometry and $E(b-y)$ data from Anthony-Twarog \& Twarog (1998) where available, including their summary of spectroscopic metallicity estimates.

ATT provided estimates of reddening, absolute magnitude, and metallicity, but we also require temperatures and gravities to make comparisons with models. For this we require relations between colors and effective temperature, $T_{\text {eff, }}$ and bolometric corrections, $\mathrm{BC}_{V}$. We discuss the derivations of these quantities below. Table 1 summarizes the photometry employed in our work. The $V-K$ values are on the "TCS" system (see Alonso, Arribas, \& Martínez Roger 1994 ), while the $V-R$ values are on the Johnson system and the $R-I$ photometry is on the Cousins system. We were able to find infrared photometry for roughly half our program stars in the second release of the 2MASS Point Source Cata$\log { }^{1}$ We transformed the 2MASS $K$ magnitudes to the "CIT" system following the prescription given in the Explanatory Supplement to the 2MASS Second Incremental Data Release, followed by a transformation to the TCS

1 The 2MASS photometry discussed here is a product of the Two Micron All Sky Survey, which is a joint project of the University of Massachusetts and the Infrared Processing and Analysis Center, California Institute of Technology, funded by the National Aeronautics and Space Administration and the National Science Foundation. 
TABLE 1

Photometry of Program Stars

\begin{tabular}{|c|c|c|c|c|c|c|c|c|c|}
\hline Star & $E(b-y)$ & $b-y$ & Ref. & $V-K$ & Ref. & $V-R_{\mathrm{J}}$ & Ref. & $(R-I)_{\mathrm{C}}$ & Ref. \\
\hline HD $20 .$. & 0.006 & 0.435 & 1 & 1.825 & 3 & & & $\ldots$ & \\
\hline 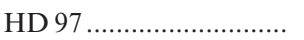 & 0.000 & 0.514 & 1 & 2.16 & 4 & 0.684 & 11 & $\ldots$ & \\
\hline $\mathrm{CD}-23^{\circ} 72 \ldots \ldots \ldots \ldots \ldots$ & 0.012 & 0.459 & 1 & 1.92 & 4 & $\ldots$ & & $\cdots$ & \\
\hline 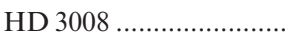 & 0.012 & 0.852 & 1 & 3.07 & 4 & 1.036 & 11 & $\ldots$ & \\
\hline HD 3179 & 0.007 & 0.451 & 1 & 1.91 & 4 & $\ldots$ & & $\ldots$ & \\
\hline $\mathrm{CD}-38^{\circ} 245 \ldots \ldots \ldots \ldots \ldots$ & 0.005 & 0.594 & 1 & 2.36 & 3 & $\ldots$ & & 0.505 & 13 \\
\hline $\mathrm{BD}-11^{\circ} 145 \ldots \ldots \ldots \ldots \ldots$ & 0.024 & 0.560 & 1 & $\ldots$ & & 0.771 & 11 & $\ldots$ & \\
\hline HD $5426 \ldots \ldots \ldots \ldots \ldots \ldots \ldots$ & 0.002 & 0.505 & 1 & 2.08 & 6 & $\ldots$ & & $\ldots$ & \\
\hline $\mathrm{BD}-20^{\circ} 170 \ldots \ldots \ldots \ldots \ldots \ldots$ & 0.014 & 0.485 & 1 & 1.99 & 4 & 0.650 & 11 & $\ldots$ & \\
\hline $\mathrm{CD}-30^{\circ} 298 \ldots \ldots \ldots \ldots \ldots$ & 0.000 & 0.484 & 1 & & & & & $\ldots$ & \\
\hline 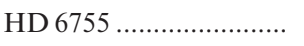 & 0.024 & 0.498 & 1 & 2.08 & $7,8,9$ & 0.676 & 11 & $\ldots$ & \\
\hline 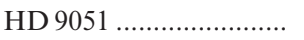 & 0.008 & 0.540 & 1 & 2.27 & 4 & $\ldots$ & & $\ldots$ & \\
\hline $\mathrm{BD}-18^{\circ} 271 \ldots \ldots \ldots \ldots \ldots \ldots$ & 0.004 & 0.846 & 1 & 3.03 & 4 & 1.030 & 11 & $\ldots$ & \\
\hline HD 13359 & 0.000 & $\ldots$ & & 1.90 & 6 & $\ldots$ & & 0.419 & 14 \\
\hline $\mathrm{BD}-22^{\circ} 395 \ldots \ldots \ldots \ldots \ldots$ & 0.000 & 0.554 & 1 & 2.27 & 4 & 0.736 & 11 & $\ldots$ & \\
\hline $\mathrm{BD}+44^{\circ} 493 \ldots \ldots \ldots \ldots \ldots$ & 0.047 & 0.454 & 1 & 1.90 & 3 & & & & \\
\hline $\mathrm{BD}-10^{\circ} 548 \ldots \ldots \ldots \ldots \ldots$ & 0.001 & 0.513 & 1 & 2.17 & 4 & 0.706 & 11 & 0.445 & 14 \\
\hline $\mathrm{CD}-30^{\circ} 1121 \ldots \ldots \ldots \ldots \ldots$ & 0.011 & 0.517 & 1 & $\ldots$ & & $\ldots$ & & $\ldots$ & \\
\hline 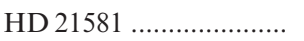 & 0.037 & 0.564 & 1 & 2.30 & 9 & 0.750 & 11 & 0.494 & 14 \\
\hline 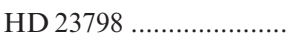 & 0.000 & 0.740 & 1 & 2.80 & 6 & $\ldots$ & & 0.588 & 14 \\
\hline 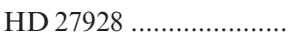 & 0.002 & 0.496 & 1 & 2.11 & 6 & $\ldots$ & & 0.446 & 14 \\
\hline 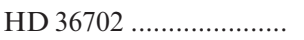 & 0.008 & 0.826 & 1 & & & $\ldots$ & & 0.625 & 14 \\
\hline $\mathrm{BD}-19^{\circ} 1422 \ldots \ldots \ldots \ldots \ldots$ & 0.022 & $\ldots$ & & 2.35 & 6 & 0.725 & 12 & 0.495 & 14 \\
\hline 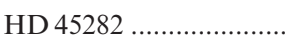 & 0.013 & 0.464 & 1 & 1.93 & 7,9 & 0.605 & 9 & 0.412 & 14 \\
\hline HD 63791 ......................... & 0.022 & 0.611 & 2 & & & 0.798 & 11 & $\ldots$ & \\
\hline 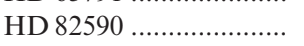 & 0.029 & 0.338 & 1 & 1.49 & 4 & 0.511 & 11 & 0.321 & 14 \\
\hline 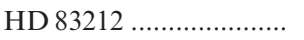 & 0.018 & 0.694 & 1 & 2.74 & 4 & 0.877 & 11 & 0.573 & 14 \\
\hline $\mathrm{BD}+30^{\circ} 2034 \ldots \ldots \ldots \ldots \ldots$ & 0.000 & 0.772 & 3 & 2.80 & 3 & & & & \\
\hline $\mathrm{CD}-30^{\circ} 8626 \ldots \ldots \ldots \ldots \ldots$ & 0.034 & 0.521 & 1 & $\ldots$ & & 0.69 & 12 & 0.474 & 14 \\
\hline 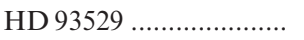 & 0.048 & 0.582 & 1 & 2.42 & 4 & 0.75 & 12 & 0.508 & 14 \\
\hline $\mathrm{BD}+22^{\circ} 2411 \ldots$ & 0.013 & 0.770 & 1 & 2.80 & 4 & 0.947 & 11 & $\ldots$ & \\
\hline $\mathrm{BD}-1^{\circ} 2582 \ldots \ldots \ldots \ldots \ldots \ldots$ & 0.004 & 0.475 & 1 & 1.99 & 4 & 0.634 & 11 & $\ldots$ & \\
\hline 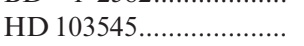 & 0.000 & 0.58 & 1 & 2.41 & 4 & 0.765 & 11 & $\ldots$ & \\
\hline $\mathrm{BD}+9^{\circ} 2574 \ldots \ldots \ldots \ldots$ & 0.000 & 0.530 & 1 & 2.20 & 4 & 0.698 & 11 & $\cdots$ & \\
\hline 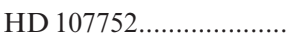 & 0.000 & 0.578 & 1 & $\ldots$ & & 0.753 & 11 & $\ldots$ & \\
\hline 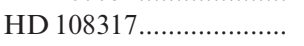 & 0.000 & 0.450 & 4 & 1.905 & 4 & 0.605 & 11 & $\ldots$ & \\
\hline 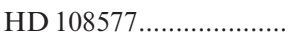 & 0.015 & 0.506 & 1 & 2.10 & 4 & 0.670 & 11 & $\ldots$ & \\
\hline HD $110281 \ldots \ldots \ldots \ldots \ldots \ldots$ & 0.004 & 1.045 & 1 & 3.56 & 4 & $\ldots$ & & 0.764 & 15 \\
\hline 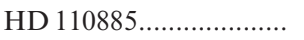 & 0.000 & 0.423 & 1 & $\ldots$ & & 0.574 & 11 & $\ldots$ & \\
\hline 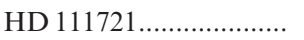 & 0.008 & 0.526 & 1 & 2.19 & 7 & $\ldots$ & & 0.479 & 14 \\
\hline $\mathrm{BD}+10^{\circ} 2495 \ldots \ldots \ldots \ldots \ldots$ & 0.002 & 0.522 & 1 & 2.14 & 4 & 0.678 & 11 & $\ldots$ & \\
\hline $\mathrm{BD}+12^{\circ} 2547 \ldots \ldots \ldots \ldots \ldots$ & 0.004 & 0.635 & 1 & $\ldots$ & & 0.784 & 11 & $\ldots$ & \\
\hline $\mathrm{BD}+3^{\circ} 2782 \ldots \ldots \ldots \ldots \ldots \ldots$ & 0.000 & & & $\ldots$ & & 0.832 & 11 & $\ldots$ & \\
\hline 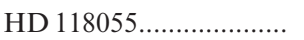 & 0.038 & 0.852 & 1 & $\ldots$ & & 1.019 & 11 & 0.670 & 14 \\
\hline $\mathrm{BD}+18^{\circ} 2757 \ldots \ldots \ldots \ldots \ldots$ & 0.000 & 0.550 & 1 & 2.255 & 4 & 0.699 & 11 & $\ldots$ & \\
\hline 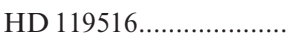 & 0.000 & 0.410 & 1 & $\ldots$ & & 0.551 & 11 & $\ldots$ & \\
\hline $\mathrm{CD}-33^{\circ} 9314 \ldots \ldots \ldots \ldots \ldots$ & 0.000 & $\ldots$ & & $\ldots$ & & 0.69 & 12 & 0.480 & 14 \\
\hline 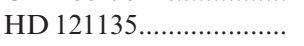 & 0.008 & 0.530 & 1 & 2.17 & 4 & 0.685 & 11 & $\ldots$ & \\
\hline 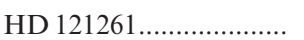 & 0.042 & 0.823 & 1 & $\ldots$ & & 1.01 & 12 & 0.660 & 14 \\
\hline HD $122956 \ldots \ldots \ldots \ldots \ldots \ldots$ & 0.042 & 0.667 & 1 & 2.70 & 6 & 0.846 & 11 & 0.589 & 14 \\
\hline 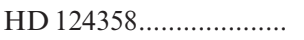 & 0.031 & 0.630 & 1 & 2.51 & 4 & 0.803 & 11 & 0.543 & 14 \\
\hline $\mathrm{BD}+9^{\circ} 2860 \ldots \ldots \ldots \ldots \ldots$ & 0.003 & 0.440 & 1 & 1.90 & 4 & 0.606 & 11 & $\ldots$ & \\
\hline $\mathrm{BD}+9^{\circ} 2870 \ldots \ldots \ldots \ldots \ldots \ldots$ & 0.012 & 0.647 & 1 & $\ldots$ & & 0.812 & 11 & $\ldots$ & \\
\hline $\mathrm{BD}+8^{\circ} 2856 \ldots \ldots \ldots \ldots \ldots$ & 0.004 & 0.685 & 1 & 2.69 & 4 & 0.834 & 11 & $\ldots$ & \\
\hline 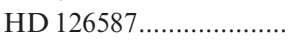 & 0.058 & 0.596 & 1 & 2.42 & 4 & 0.76 & 12 & 0.540 & 14 \\
\hline $\mathrm{BD}+18^{\circ} 2890 \ldots \ldots \ldots \ldots \ldots$ & 0.000 & 0.505 & 5 & 2.11 & 4 & 0.656 & 11 & $\ldots$ & \\
\hline $\mathrm{BD}-8^{\circ} 3901 \ldots \ldots \ldots \ldots \ldots \ldots$ & 0.029 & $\ldots$ & & $\ldots$ & & $\ldots$ & & 0.536 & 14 \\
\hline $\mathrm{BD}+18^{\circ} 2976 \ldots \ldots \ldots \ldots \ldots \ldots$ & 0.005 & 0.655 & 1 & 2.59 & 6 & 0.810 & 11 & $\ldots$ & \\
\hline 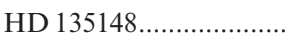 & 0.016 & 0.869 & 1 & 3.04 & 6 & 0.987 & 11 & $\ldots$ & \\
\hline $\mathrm{BD}+1^{\circ} 3070 \ldots \ldots \ldots \ldots \ldots$ & 0.016 & 0.487 & 1 & $\ldots$ & & 0.636 & 11 & $\ldots$ & \\
\hline 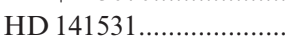 & 0.012 & 0.765 & 1 & 2.76 & 4 & 0.923 & 11 & $\ldots$ & \\
\hline $\mathrm{BD}+5^{\circ} 3098 \ldots \ldots \ldots \ldots \ldots$ & 0.028 & 0.542 & 1 & $\ldots$ & & 0.708 & 11 & $\ldots$ & \\
\hline $\mathrm{BD}+11^{\circ} 2998 \ldots \ldots \ldots \ldots \ldots$ & 0.035 & 0.453 & 1 & $\ldots$ & & 0.606 & 11 & $\ldots$ & \\
\hline $\mathrm{BD}+9^{\circ} 3223 \ldots \ldots \ldots \ldots \ldots$ & 0.041 & 0.469 & 1 & 1.97 & 4 & 0.625 & 11 & $\ldots$ & \\
\hline $\mathrm{BD}+13^{\circ} 3683 \ldots \ldots \ldots \ldots \ldots$ & 0.092 & 0.507 & 1 & 2.07 & 9 & $\ldots$ & & 0.46 & 16 \\
\hline
\end{tabular}


TABLE 1-Continued

\begin{tabular}{|c|c|c|c|c|c|c|c|c|c|}
\hline Star & $E(b-y)$ & $b-y$ & Ref. & $V-K$ & Ref. & $V-R_{\mathrm{J}}$ & Ref. & $(R-I)_{\mathrm{C}}$ & Ref. \\
\hline HD $171496 \ldots \ldots \ldots \ldots \ldots \ldots . . . . . . .$. & 0.189 & 0.764 & 1 & 2.91 & 4 & $\cdots$ & & 0.639 & 14 \\
\hline HD $175305 \ldots \ldots \ldots \ldots \ldots . . . . . . . .$. & 0.020 & 0.508 & 1 & 2.11 & 9 & 0.666 & 11 & $\ldots$ & \\
\hline HD $176203 \ldots \ldots \ldots \ldots \ldots \ldots . . . . . .$. & 0.000 & $\ldots$ & & 2.15 & 6 & $\ldots$ & & 0.484 & 14 \\
\hline HD $181007 \ldots \ldots \ldots \ldots \ldots \ldots$ & 0.014 & $\ldots$ & & 2.33 & 10 & 0.725 & 9 & 0.501 & 14 \\
\hline 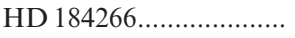 & 0.039 & 0.427 & 1 & 1.78 & 9 & 0.596 & 11 & 0.406 & 14 \\
\hline HD $186478 \ldots \ldots \ldots$ & 0.057 & 0.689 & 1 & 2.73 & 6 & 0.893 & 11 & 0.596 & 14 \\
\hline $\mathrm{BD}-18^{\circ} 5550 \ldots \ldots \ldots \ldots \ldots$ & 0.086 & 0.685 & 1 & 2.74 & 9 & 0.885 & 11 & 0.519 & 14 \\
\hline HD $193242 \ldots \ldots \ldots \ldots \ldots \ldots . . . . . . .$. & 0.000 & $\cdots$ & & 2.32 & 6 & $\cdots$ & & 0.485 & 14 \\
\hline HD $195636 \ldots \ldots .$. & 0.044 & 0.467 & 1 & 1.955 & 3 & 0.634 & 11 & $\ldots$ & \\
\hline $\mathrm{BD}-17^{\circ} 6036 \ldots \ldots \ldots \ldots \ldots$ & 0.036 & 0.581 & 1 & 2.37 & 4 & 0.760 & 11 & $\ldots$ & \\
\hline $\mathrm{BD}-20^{\circ} 6008 \ldots \ldots \ldots \ldots . . . .$. & 0.014 & $\ldots$ & & $\ldots$ & & $\ldots$ & & 0.541 & 14 \\
\hline $\mathrm{BD}-15^{\circ} 5781 \ldots \ldots \ldots \ldots \ldots$ & 0.020 & 0.601 & 1 & 2.83 & 4 & 0.801 & 11 & $\ldots$ & \\
\hline $\mathrm{BD}-14^{\circ} 5890 \ldots \ldots \ldots \ldots \ldots$ & 0.023 & 0.554 & 1 & $\ldots$ & & 0.734 & 11 & $\ldots$ & \\
\hline $\mathrm{CD}-37^{\circ} 14010 \ldots \ldots \ldots \ldots$ & 0.000 & $\ldots$ & & $\ldots$ & & $\cdots$ & & 0.582 & 14 \\
\hline $\mathrm{BD}-3^{\circ} 5215 \ldots \ldots \ldots \ldots \ldots$ & 0.032 & 0.435 & 1 & 1.82 & 3 & 0.598 & 11 & $\ldots$ & \\
\hline 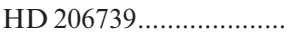 & 0.023 & 0.625 & 1 & 2.53 & 4 & 0.814 & 11 & $\ldots$ & \\
\hline $\mathrm{BD}-9^{\circ} 5831 \ldots \ldots \ldots \ldots \ldots \ldots$ & 0.017 & 0.645 & 1 & 2.60 & 4 & 0.835 & 11 & $\ldots$ & \\
\hline HD $208069 . . .$. & 0.000 & 0.479 & 1 & 2.05 & 6 & $\ldots$ & & 0.442 & 14 \\
\hline 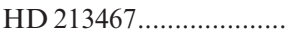 & 0.000 & 0.484 & 1 & $\ldots$ & & $\ldots$ & & 0.439 & 14 \\
\hline HD $214362 \ldots \ldots \ldots \ldots \ldots \ldots$ & 0.006 & 0.370 & 1 & 1.59 & 3 & $\ldots$ & & 0.353 & 14 \\
\hline HD $214925 \ldots \ldots \ldots \ldots \ldots \ldots$ & 0.000 & $\ldots$ & & 3.40 & 6 & $\ldots$ & & 0.724 & 14 \\
\hline HD $218732 \ldots \ldots \ldots \ldots \ldots \ldots . . . . . .$. & 0.022 & 1.05 & 5 & 3.60 & 6 & 1.209 & 11 & $\ldots$ & \\
\hline HD $220662 \ldots \ldots \ldots \ldots \ldots \ldots . . . . . .$. & 0.006 & 0.684 & 1 & 2.67 & 4 & $\ldots$ & & $\ldots$ & \\
\hline HD $220838 \ldots \ldots \ldots \ldots \ldots \ldots . . . . . . .$. & 0.007 & 0.765 & 1 & $\ldots$ & & $\ldots$ & & $\cdots$ & \\
\hline HD $221170 \ldots \ldots \ldots \ldots \ldots . . . . . . .$. & 0.043 & 0.756 & 1 & 2.86 & 9 & 0.927 & 11 & $\ldots$ & \\
\hline 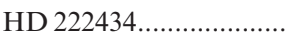 & 0.007 & 0.708 & 1 & 2.65 & 6 & $\ldots$ & & $\cdots$ & \\
\hline
\end{tabular}

REFERENCES.- (1) Anthony-Twarog \& Twarog 1994; (2) Jensen \& Olsen 1979; (3) Carney \& Latham 1986; (4) Alonso, Arribas, \& Martínez Roger 1998; (5) Bond 1980; (6) 2MASS; (7) Alonso et al. 1994; (8) Arribas \& Martínez Roger 1987; (9) Carney 1983; (10) Alonso, Arribas, \& Martínez Roger 1995; (11) Stone 1983; (12) Carney 1980; (13) Bessell \& Norris 1984; (14) Norris et al. 1985; (15) Menzies et al. 1991; (16) Ryan, Norris, \& Bessell 1991.

system as described by Alonso et al. (1994). A total of 27 stars in our program with TCS photometry are in the second release of the Point Source Catalog. We found that $\left\langle\left(V-K_{\mathrm{TCS}}\right)-\left(V-K_{2 \mathrm{MASS}}\right)\right\rangle=+0.020 \pm 0.008 \mathrm{mag}$, with $\sigma=0.044$ mag. The moderately lower precision of the 2MASS observations is the primary cause of the relatively large scatter, and we have therefore chosen to employ the 2MASS results only for the 15 stars for which no other infrared data were available. We added $0.02 \mathrm{mag}$ to the $V-K_{\mathrm{TCS}}$ values to correct for the observed small systematic offset.

Most of our program stars have $M_{V}$ and $E(b-y)$ estimates from ATT. For most of the stars lacking uvby photometry, estimates of $M_{V}$ and $E(B-V)$ have been provided by NBP. Since ATT relied on the $M_{V}$ versus $B-V$ relations as a function of metallicity derived by NBP, the absolute magnitude scales are identical. Stars for which we have adopted $M_{V}$ and $E(b-y)[=0.72 E(B-V)]$ values from NBP are noted in Table 2 .

A few of our program stars required special attention. The metallicity adopted for CD $-38^{\circ} 245$ was taken from the high-resolution spectroscopic analyses of Norris, Peterson, \& Beers (1993), Peterson, Kurucz, \& Carney (1990), and Bessell \& Norris (1984). Several stars not studied by ATT have uvby photometry, and for three of them, HD $63791, \mathrm{BD}+30^{\circ} 2034$, and $\mathrm{BD}+18^{\circ} 2890$, estimation of reddening and $M_{V}$ is straightforward, following the precepts of ATT. $[\mathrm{Fe} / \mathrm{H}]$ values for the three stars were taken directly from Kraft et al. (1992), Luck \& Bond (1985), and Pilachowski, Sneden, \& Booth (1993), respectively. BD $+3^{\circ} 2782$ was not studied by either ATT or NBP and lacks uvby photometry. Its high latitude $\left(b=+64^{\circ}\right)$ suggests zero reddening, which we have adopted. Sneden et al. (1991) have analyzed the star spectroscopically, finding $[\mathrm{Fe} / \mathrm{H}]=$ -2.01 . With this metallicity, and $B-V=0.98$, the NBP algorithm indicates $M_{V}=-1.31$. We have adopted the spectroscopic result for $[\mathrm{Fe} / \mathrm{H}]$. The uvby photometry of HD 218732 is almost identical to that of CK Vir, for which Leep \& Wallerstein (1981) have estimated $T_{\text {eff }}=4000 \mathrm{~K}$, $\log g=0.0$, and $[\mathrm{Fe} / \mathrm{H}]=-2.8$. This star is very near the red giant branch (RGB) tip, and we adopt $M_{V}=-2.8$. The Galactic latitude of the star is $-63^{\circ}$, and we adopt $E(B-V)=0.03 \mathrm{mag}$, as ATT did for the south Galactic pole.

In some cases, our derived stellar parameters are vulnerable to additional uncertainties. We have noted the following stars in Table 2: BD $-1^{\circ} 2582$ (Bond 1980) and HD 135148 (Bond 1980; Shetrone et al. 1999) are CH-strong stars, and ATT noted that the $m_{1}$ index and photometric metallicity estimate may be affected. We relied on the spectroscopic metallicities reported by ATT and rederived the reddening and $M_{V}$ values according to their prescription. ATT raised the possibility of poor photometry for HD 126587 or that the reddening estimate is wrong or it is a $\mathrm{CH}$-strong or $\mathrm{CN}$-strong star. We have relied on the NBP estimates for reddening and $M_{V}$. BD $+44^{\circ} 493$ lies at low Galactic latitude, and hence its reddening is less certain, and as we discuss in $\S 4.4$, a better match between the observed and synthetic spectra is obtained if the star's temperature is higher than we have estimated via the photometry. However, none of our conclusions regarding the star's derived radial velocities or line broadening are affected. 
TABLE 2

Stellar Parameters

\begin{tabular}{|c|c|c|c|c|c|c|c|c|c|}
\hline Star & $\alpha(\mathrm{J} 2000)$ & $\delta(\mathrm{J} 2000)$ & {$[\mathrm{Fe} / \mathrm{H}]$} & $M_{V}$ & $\begin{array}{l}T_{\text {eff }} \\
(\mathrm{K})\end{array}$ & $\log g$ & $\begin{array}{c}R \\
\left(R_{\odot}\right)\end{array}$ & $\begin{array}{c}d \\
(\mathrm{pc})\end{array}$ & Template \\
\hline HD $20 \ldots .$. & 000515.3 & -271618 & -1.66 & 0.7 & 5350 & 2.5 & 8.2 & 460 & at $5250 \mathrm{~g} 25 \mathrm{~m} 15 \mathrm{v} 006$ \\
\hline HD 97 & 000546.0 & -194012 & -1.38 & 1.3 & 4910 & 2.6 & 7.9 & 470 & at $5000 \mathrm{~g} 25 \mathrm{~m} 15 \mathrm{v} 004$ \\
\hline $\mathrm{CD}-23^{\circ} 72 \ldots \ldots \ldots \ldots \ldots$ & 001616.5 & -223440 & -1.12 & 0.8 & 5270 & 2.5 & 8.0 & 550 & at $5250 \mathrm{~g} 25 \mathrm{~m} 10 \mathrm{v} 008$ \\
\hline HD $3008 \ldots \ldots \ldots \ldots . . . .$. & 003314.2 & -104343 & -1.43 & -1.5 & 4140 & 1.0 & 49.5 & 1610 & at $4250 \mathrm{~g} 10 \mathrm{~m} 15 \mathrm{v} 010$ \\
\hline HD 3179 . & 003450.6 & -215256 & -0.92 & 0.9 & 5280 & 2.6 & 7.8 & 590 & at $5250 \mathrm{~g} 25 \mathrm{~m} 10 \mathrm{v} 006$ \\
\hline $\mathrm{CD}-38^{\circ} 245^{\mathrm{a}}$. & 004636.1 & -373933 & -4.00 & -0.7 & 4920 & 1.8 & 19.8 & 3360 & at $5000 \mathrm{~g} 20 \mathrm{~m} 30 \mathrm{v} 004$ \\
\hline $\mathrm{BD}-11^{\circ} 145 .$. & 004824.3 & -104130 & -2.02 & -0.6 & 4780 & 1.7 & 20.5 & 1680 & at $4750 \mathrm{~g} 15 \mathrm{~m} 20 \mathrm{v} 006$ \\
\hline 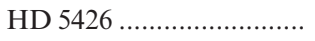 & 005540.9 & -334510 & -2.33 & 0.3 & 4990 & 2.2 & 11.7 & 720 & at $5000 \mathrm{~g} 20 \mathrm{~m} 25 \mathrm{v} 006$ \\
\hline $\mathrm{BD}-20^{\circ} 170 \ldots \ldots \ldots \ldots \ldots \ldots$ & 005710.2 & -194957 & -1.31 & 0.8 & 5130 & 2.5 & 8.7 & 780 & at $5250 \mathrm{~g} 25 \mathrm{~m} 15 \mathrm{v} 006$ \\
\hline $\mathrm{CD}-30^{\circ} 298 \ldots \ldots$ & 005843.7 & -300558 & -2.90 & 0.8 & 5120 & 2.4 & 8.9 & 950 & at $5000 \mathrm{~g} 25 \mathrm{~m} 30 \mathrm{v} 004$ \\
\hline 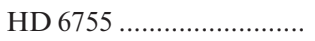 & 010943.0 & +613250 & -1.72 & 1.5 & 5080 & 2.7 & 6.6 & 170 & at $5000 \mathrm{~g} 25 \mathrm{~m} 15 \mathrm{v} 004$ \\
\hline 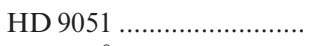 & 012846.5 & -242025 & -1.50 & 0.7 & 4840 & 2.3 & 10.9 & 440 & at $4750 \mathrm{~g} 25 \mathrm{~m} 15 \mathrm{v} 000$ \\
\hline $\mathrm{BD}-18^{\circ} 271 \ldots \ldots$. & 013718.8 & -172903 & -1.98 & -2.1 & 4150 & 0.7 & 63.0 & 2420 & at $4250 \mathrm{~g} 05 \mathrm{~m} 20 \mathrm{v} 008$ \\
\hline HD $13359^{\mathrm{b}} \ldots \ldots \ldots \ldots \ldots \ldots \ldots \ldots$ & 021007.0 & -204551 & -1.66 & 1.7 & 5150 & 2.8 & 5.9 & 410 & at $5250 \mathrm{~g} 30 \mathrm{~m} 15 \mathrm{v} 008$ \\
\hline $\mathrm{BD}-22^{\circ} 395 \ldots \ldots \ldots \ldots \ldots$ & 022157.8 & -213042 & -2.14 & -0.6 & 4780 & 1.7 & 20.4 & 1700 & at $4750 \mathrm{~g} 15 \mathrm{~m} 20 \mathrm{v} 006$ \\
\hline $\mathrm{BD}+44^{\circ} 493^{\mathrm{c}}$ & 022649.7 & +445746 & -2.71 & 0.8 & 5510 & 2.6 & 7.4 & 420 & at $5500 \mathrm{~g} 25 \mathrm{~m} 25 \mathrm{v} 004$ \\
\hline $\mathrm{BD}-10^{\circ} 548 .$. & 024655.6 & -100735 & -1.71 & 0.8 & 4900 & 2.4 & 9.9 & 830 & at $5000 \mathrm{~g} 25 \mathrm{~m} 15 \mathrm{v} 002$ \\
\hline $\mathrm{CD}-30^{\circ} 1121$. & 025700.2 & -302437 & -1.82 & 0.8 & 4940 & 2.4 & 9.5 & 770 & at $5000 \mathrm{~g} 25 \mathrm{~m} 20 \mathrm{v} 002$ \\
\hline HD $21581 \ldots \ldots$. & 032854.4 & -002502 & -1.65 & 0.6 & 4860 & 2.3 & 11.0 & 390 & at $4750 \mathrm{~g} 25 \mathrm{~m} 15 \mathrm{v} 006$ \\
\hline HD 23798 .. & 034645.7 & -305113 & -1.90 & -1.8 & 4310 & 1.0 & 48.7 & 1050 & at $4250 \mathrm{~g} 10 \mathrm{~m} 20 \mathrm{v} 004$ \\
\hline HD 27928. & 042255.0 & -371549 & -2.25 & 0.5 & 4990 & 2.3 & 10.7 & 640 & at $5000 \mathrm{~g} 25 \mathrm{~m} 20 \mathrm{v} 002$ \\
\hline HD 36702 . & 053152.1 & -383324 & -1.86 & -1.9 & 4180 & 0.8 & 57.3 & 1140 & at $4250 \mathrm{~g} 10 \mathrm{~m} 20 \mathrm{v} 006$ \\
\hline $\mathrm{BD}-19^{\circ} 1422^{\mathrm{b}}$ & 062110.5 & -191508 & -1.86 & 0.4 & 4800 & 2.2 & 12.5 & 680 & at $4750 \mathrm{~g} 20 \mathrm{~m} 20 \mathrm{v} 006$ \\
\hline HD $45282 \ldots \ldots$. & 062640.7 & +032529 & -1.80 & 1.9 & 5230 & 2.9 & 5.1 & 160 & at $5250 \mathrm{~g} 30 \mathrm{~m} 20 \mathrm{v} 004$ \\
\hline HD $63791^{\mathrm{a}, \mathrm{b}}$. & 075428.7 & +620810 & -1.64 & -0.3 & 4660 & 1.8 & 18.7 & 420 & at $4750 \mathrm{~g} 20 \mathrm{~m} 15 \mathrm{v} 004$ \\
\hline HD $82590 \ldots \ldots \ldots . . . .$. & 093230.3 & -172608 & -1.85 & 0.7 & 5960 & 2.7 & 6.5 & 530 & at $5750 \mathrm{~g} 25 \mathrm{~m} 15 \mathrm{v} 012$ \\
\hline HD 83212 . & 093619.9 & -205315 & -1.45 & -0.9 & 4430 & 1.4 & 29.5 & 690 & at $4500 \mathrm{~g} 15 \mathrm{~m} 15 \mathrm{v} 008$ \\
\hline $\mathrm{BD}+30^{\circ} 2034^{\mathrm{a}, \mathrm{b}}$. & 103314.9 & +293655 & -1.53 & -1.7 & 4290 & 1.0 & 46.9 & 2650 & at $4250 \mathrm{~g} 10 \mathrm{~m} 15 \mathrm{v} 008$ \\
\hline $\mathrm{CD}-30^{\circ} 8626 \ldots$ & 104109.2 & -304704 & -1.67 & 0.3 & 5000 & 2.2 & 12.0 & 720 & at $5000 \mathrm{~g} 20 \mathrm{~m} 15 \mathrm{v} 004$ \\
\hline HD $93529 \ldots \ldots$. & 104729.0 & -252614 & -1.24 & 1.1 & 4840 & 2.4 & 9.1 & 410 & at $4750 \mathrm{~g} 25 \mathrm{~m} 15 \mathrm{v} 002$ \\
\hline $\mathrm{BD}+22^{\circ} 2411 \ldots$. & 115133.5 & +220651 & -1.95 & -1.7 & 4320 & 1.0 & 46.8 & 2120 & at $4250 \mathrm{~g} 10 \mathrm{~m} 20 \mathrm{v} 008$ \\
\hline $\mathrm{BD}-1^{\circ} 2582^{\mathrm{c}} \ldots \ldots \ldots \ldots \ldots$ & 115337.3 & -020037 & -2.32 & 0.7 & 5130 & 2.4 & 9.4 & 610 & at $5250 \mathrm{~g} 25 \mathrm{~m} 25 \mathrm{v} 006$ \\
\hline HD $103545 \ldots$. & 115527.1 & +090743 & -2.42 & -0.7 & 4690 & 1.7 & 22.2 & 1070 & at $4750 \mathrm{~g} 15 \mathrm{~m} 25 \mathrm{v} 004$ \\
\hline $\mathrm{BD}+9^{\circ} 2574 \ldots \ldots$ & 120203.9 & +090040 & -1.95 & 0.3 & 4860 & 2.1 & 12.9 & 1030 & at $4750 \mathrm{~g} 20 \mathrm{~m} 20 \mathrm{v} 002$ \\
\hline HD $107752 \ldots \ldots . .$. & 122252.7 & +113625 & -2.64 & -0.7 & 4750 & 1.7 & 21.6 & 1410 & at $4750 \mathrm{~g} 15 \mathrm{~m} 25 \mathrm{v} 004$ \\
\hline HD $108317 .$. & 122404.4 & +053446 & -2.48 & 0.5 & 5230 & 2.4 & 9.6 & 320 & at $5250 \mathrm{~g} 25 \mathrm{~m} 25 \mathrm{v} 006$ \\
\hline HD $108577 \ldots \ldots$. & 122816.8 & +122040 & -2.50 & -0.6 & 5040 & 1.9 & 17.4 & 1050 & at $5000 \mathrm{~g} 20 \mathrm{~m} 25 \mathrm{v} 006$ \\
\hline 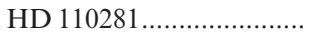 & 124103.6 & -003713 & -1.75 & -2.6 & 3850 & 0.2 & 111.6 & 2480 & at $4000 \mathrm{~g} 05 \mathrm{~m} 15 \mathrm{v} 012$ \\
\hline HD $110885 \ldots \ldots \ldots . .$. & 124519.2 & +010320 & -1.59 & 0.7 & 5330 & 2.5 & 8.2 & 480 & at $5250 \mathrm{~g} 25 \mathrm{~m} 15 \mathrm{v} 008$ \\
\hline HD $111721 \ldots \ldots$ & 124849.0 & -131254 & -1.42 & 1.1 & 4860 & 2.5 & 8.6 & 230 & at $4750 \mathrm{~g} 25 \mathrm{~m} 15 \mathrm{v} 002$ \\
\hline $\mathrm{BD}+10^{\circ} 2495 .$. & 125919.9 & +091435 & -2.14 & 0.3 & 4920 & 2.2 & 12.4 & 760 & at $5000 \mathrm{~g} 20 \mathrm{~m} 20 \mathrm{v} 004$ \\
\hline $\mathrm{BD}+12^{\circ} 2547 \ldots$ & 130406.5 & +112616 & -2.07 & -0.9 & 4610 & 1.5 & 26.4 & 1470 & at $4500 \mathrm{~g} 15 \mathrm{~m} 20 \mathrm{v} 004$ \\
\hline $\mathrm{BD}+3^{\circ} 2782^{\mathrm{a}} \ldots \ldots \ldots \ldots \ldots$ & 132955.9 & +024526 & -2.01 & -1.3 & 4500 & 1.3 & 33.6 & 1610 & at $4500 \mathrm{~g} 15 \mathrm{~m} 20 \mathrm{v} 006$ \\
\hline 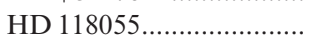 & 133439.9 & -161922 & -1.46 & -1.5 & 4160 & 1.0 & 47.2 & 1100 & at $4250 \mathrm{~g} 10 \mathrm{~m} 15 \mathrm{v} 008$ \\
\hline $\mathrm{BD}+18^{\circ} 2757 \ldots \ldots \ldots \ldots \ldots \ldots$ & 133631.9 & +180901 & -2.52 & -0.8 & 4840 & 1.7 & 21.6 & 1320 & at $4750 \mathrm{~g} 15 \mathrm{~m} 25 \mathrm{v} 008$ \\
\hline 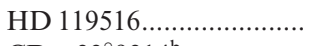 & 134326.7 & +153429 & -2.49 & 0.6 & 5440 & 2.5 & 8.6 & 520 & at $5500 \mathrm{~g} 25 \mathrm{~m} 25 \mathrm{v} 010$ \\
\hline $\mathrm{CD}-33^{\circ} 9314^{\mathrm{b}}$. & 134603.4 & -335625 & -2.10 & 0.0 & 4820 & 2.0 & 15.3 & 1040 & at $4750 \mathrm{~g} 20 \mathrm{~m} 20 \mathrm{v} 004$ \\
\hline HD $121135 \ldots \ldots . .$. & 135333.4 & +024141 & -1.83 & -0.4 & 4910 & 1.9 & 16.8 & 890 & at $5000 \mathrm{~g} 20 \mathrm{~m} 20 \mathrm{v} 008$ \\
\hline 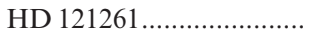 & 135504.9 & -360819 & -1.52 & -1.5 & 4210 & 1.0 & 45.7 & 1270 & at $4250 \mathrm{~g} 10 \mathrm{~m} 15 \mathrm{v} 008$ \\
\hline 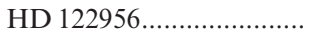 & 140513.0 & -145125 & -1.71 & -0.7 & 4530 & 1.6 & 24.8 & 350 & at $4500 \mathrm{~g} 15 \mathrm{~m} 15 \mathrm{v} 006$ \\
\hline $\mathrm{BD}+9^{\circ} 2860 \ldots \ldots \ldots \ldots \ldots \ldots$ & 141319.7 & +083640 & -1.67 & 0.7 & 5240 & 2.5 & 8.6 & 1000 & at5250g $25 \mathrm{~m} 15 \mathrm{v} 004$ \\
\hline HD $124358 \ldots$ & 141321.2 & -120922 & -1.98 & -0.9 & 4640 & 1.5 & 25.2 & 1140 & at $4750 \mathrm{~g} 15 \mathrm{~m} 20 \mathrm{v} 006$ \\
\hline $\mathrm{BD}+9^{\circ} 2870 \ldots$ & 141629.9 & +082753 & -2.37 & -1.3 & 4600 & 1.4 & 31.0 & 1360 & at $4500 \mathrm{~g} 15 \mathrm{~m} 25 \mathrm{v} 006$ \\
\hline $\mathrm{BD}+8^{\circ} 2856 \ldots \ldots \ldots \ldots \ldots \ldots$ & 142358.1 & +080133 & -2.31 & -1.9 & 4480 & 1.1 & 44.8 & 2330 & at $4500 \mathrm{~g} 10 \mathrm{~m} 25 \mathrm{v} 008$ \\
\hline 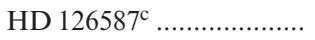 & 142700.3 & -221439 & -2.58 & -0.6 & 4850 & 1.8 & 19.4 & 790 & at $4750 \mathrm{~g} 20 \mathrm{~m} 25 \mathrm{v} 004$ \\
\hline $\mathrm{BD}+18^{\circ} 2890^{\mathrm{a}, \mathrm{b}} \ldots \ldots \ldots \ldots \ldots$ & 143213.5 & +172525 & -1.61 & 1.0 & 4970 & 2.5 & 8.6 & 560 & at $5000 \mathrm{~g} 25 \mathrm{~m} 15 \mathrm{v} 006$ \\
\hline $\mathrm{BD}-8^{\circ} 3901^{\mathrm{b}} \ldots$. & 150451.2 & -084857 & -1.88 & -0.1 & 4600 & 1.9 & 17.7 & 750 & at $4500 \mathrm{~g} 20 \mathrm{~m} 20 \mathrm{v} 004$ \\
\hline $\mathrm{BD}+18^{\circ} 2976 \ldots \ldots \ldots \ldots \ldots$ & 150724.2 & +172658 & -2.42 & -1.3 & 4550 & 1.3 & 32.7 & 1700 & at $4500 \mathrm{~g} 15 \mathrm{~m} 25 \mathrm{v} 006$ \\
\hline HD $135148^{\mathrm{c}} \ldots \ldots \ldots \ldots \ldots \ldots$ & 151317.4 & +122725 & -1.88 & -2.1 & 4180 & 0.8 & 60.5 & 1880 & at $4250 \mathrm{~g} 10 \mathrm{~m} 20 \mathrm{v} 008$ \\
\hline $\mathrm{BD}+1^{\circ} 3070 \ldots \ldots \ldots \ldots \ldots \ldots$ & 152240.0 & +011552 & -1.85 & 1.5 & 5130 & 2.7 & 6.5 & 490 & at $5000 \mathrm{~g} 25 \mathrm{~m} 20 \mathrm{v} 004$ \\
\hline 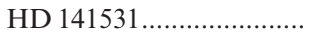 & 154916.5 & +093642 & -1.57 & -1.5 & 4340 & 1.1 & 40.5 & 1290 & at $4250 \mathrm{~g} 10 \mathrm{~m} 15 \mathrm{v} 008$ \\
\hline $\mathrm{BD}+5^{\circ} 3098 \ldots \ldots \ldots \ldots \ldots \ldots$ & 155217.3 & +051744 & -2.40 & 0.0 & 4930 & 2.0 & 14.4 & 1160 & at $5000 \mathrm{~g} 20 \mathrm{~m} 25 \mathrm{v} 006$ \\
\hline $\mathrm{BD}+11^{\circ} 2998 \ldots \ldots \ldots \ldots \ldots \ldots$ & 163016.7 & +105951 & -1.46 & 0.8 & 5360 & 2.5 & 8.0 & 430 & at $5250 \mathrm{~g} 25 \mathrm{~m} 15 \mathrm{v} 006$ \\
\hline $\mathrm{BD}+9^{\circ} 3223 \ldots \ldots \ldots \ldots \ldots$ & 163335.6 & +090617 & -2.41 & 0.6 & 5310 & 2.4 & 8.9 & 500 & at $5250 \mathrm{~g} 25 \mathrm{~m} 25 \mathrm{v} 004$ \\
\hline
\end{tabular}


TABLE 2-Continued

\begin{tabular}{|c|c|c|c|c|c|c|c|c|c|}
\hline Star & $\alpha(\mathrm{J} 2000)$ & $\delta(\mathbf{J} 2000)$ & {$[\mathrm{Fe} / \mathrm{H}]$} & $M_{V}$ & $\begin{array}{l}T_{\text {eff }} \\
(\mathrm{K})\end{array}$ & $\log g$ & $\begin{array}{c}R \\
\left(R_{\odot}\right)\end{array}$ & $\begin{array}{c}d \\
(\mathrm{pc})\end{array}$ & Template \\
\hline $\mathrm{BD}+13^{\circ} 3683 \ldots$ & 183316.8 & +130925 & -1.90 & 2.0 & 5540 & 3.1 & 4.3 & 430 & at $5500 \mathrm{~g} 30 \mathrm{~m} 20 \mathrm{v} 006$ \\
\hline HD $171496 \ldots$. & 183607.5 & -242611 & -1.16 & 0.8 & 4820 & 2.3 & 10.6 & 250 & at $4750 \mathrm{~g} 25 \mathrm{~m} 10 \mathrm{v} 008$ \\
\hline HD $175305 .$. & 184849.0 & +744001 & -1.39 & 1.8 & 5050 & 2.8 & 5.8 & 120 & at $5000 \mathrm{~g} 30 \mathrm{~m} 15 \mathrm{v} 001$ \\
\hline HD $176203^{b}$. & 190004.1 & -230348 & -1.81 & 0.9 & 4820 & 2.4 & 9.8 & 370 & at $4750 \mathrm{~g} 25 \mathrm{~m} 20 \mathrm{v} 006$ \\
\hline HD $181007^{\mathrm{b}}$ & 191928.4 & -202539 & -2.00 & 0.0 & 4770 & 2.0 & 15.6 & 810 & at $4750 \mathrm{~g} 20 \mathrm{~m} 20 \mathrm{v} 004$ \\
\hline 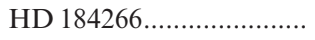 & 193415.3 & -161900 & -1.87 & 0.7 & 5490 & 2.6 & 7.9 & 220 & at $5500 \mathrm{~g} 25 \mathrm{~m} 20 \mathrm{v} 012$ \\
\hline HD $186478 \ldots \ldots$. & 194514.2 & -172926 & -2.45 & -1.1 & 4540 & 1.4 & 30.2 & 1020 & at $4500 \mathrm{~g} 15 \mathrm{~m} 25 \mathrm{v} 006$ \\
\hline $\mathrm{BD}-18^{\circ} 5550 \ldots \ldots$ & 195849.7 & -181211 & -3.11 & -0.4 & 4820 & 1.8 & 18.4 & 730 & at $4750 \mathrm{~g} 20 \mathrm{~m} 30 \mathrm{v} 004$ \\
\hline HD $193242^{b} \ldots \ldots$. & 201954.3 & -195445 & -2.22 & 0.1 & 4740 & 2.0 & 15.0 & 650 & at $4750 \mathrm{~g} 20 \mathrm{~m} 20 \mathrm{v} 006$ \\
\hline HD $195636 \ldots$ & 203248.9 & -092152 & -2.77 & 0.5 & 5370 & 2.4 & 9.1 & 600 & at $5250 \mathrm{~g} 25 \mathrm{~m} 30 \mathrm{v} 020$ \\
\hline $\mathrm{BD}-17^{\circ} 6036 \ldots$ & 203715.9 & -163357 & -2.54 & -0.2 & 4830 & 1.9 & 16.1 & 1270 & at $4750 \mathrm{~g} 20 \mathrm{~m} 25 \mathrm{v} 004$ \\
\hline $\mathrm{BD}-20^{\circ} 6008^{\mathrm{b}}$. & 204248.7 & -200039 & -2.63 & -1.3 & 4550 & 1.3 & 32.1 & 1660 & at $4500 \mathrm{~g} 15 \mathrm{~m} 25 \mathrm{v} 000$ \\
\hline $\mathrm{BD}-15^{\circ} 5781 \ldots$ & 204534.5 & -143115 & -2.47 & -0.8 & 4590 & 1.6 & 24.7 & 1970 & at $4500 \mathrm{~g} 15 \mathrm{~m} 25 \mathrm{v} 004$ \\
\hline $\mathrm{BD}-14^{\circ} 5890 \ldots$ & 205609.1 & -133116 & -2.01 & 0.3 & 4840 & 2.1 & 13.0 & 960 & at $4750 \mathrm{~g} 20 \mathrm{~m} 20 \mathrm{v} 002$ \\
\hline $\mathrm{CD}-37^{\circ} 14010$. & 205727.3 & -363251 & -2.55 & -1.9 & 4300 & 0.9 & 50.0 & 2080 & at $4250 \mathrm{~g} 10 \mathrm{~m} 25 \mathrm{v} 020$ \\
\hline $\mathrm{BD}-3^{\circ} 5215 \ldots$ & 212801.3 & -030740 & -1.64 & 0.7 & 5420 & 2.6 & 7.9 & 710 & at $5500 \mathrm{~g} 25 \mathrm{~m} 15 \mathrm{v} 008$ \\
\hline HD $206739 \ldots .$. & 214423.9 & -114622 & -1.57 & -0.3 & 4620 & 1.8 & 19.6 & 570 & at $4500 \mathrm{~g} 20 \mathrm{~m} 15 \mathrm{v} 006$ \\
\hline $\mathrm{BD}-9^{\circ} 5831 \ldots \ldots \ldots \ldots \ldots$ & 214530.5 & -082435 & -1.87 & -1.1 & 4550 & 1.4 & 28.8 & 1880 & at $4500 \mathrm{~g} 15 \mathrm{~m} 20 \mathrm{v} 006$ \\
\hline HD $208069 \ldots$ & 215426.0 & -301524 & -1.83 & 1.3 & 5030 & 2.6 & 7.5 & 390 & at $5000 \mathrm{~g} 25 \mathrm{~m} 20 \mathrm{v} 004$ \\
\hline HD $213467 \ldots$ & 223207.9 & -311025 & -1.45 & 1.7 & 5010 & 2.8 & 6.1 & 240 & at $5000 \mathrm{~g} 30 \mathrm{~m} 15 \mathrm{v} 001$ \\
\hline 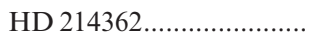 & 223758.6 & -223840 & -2.20 & 0.6 & 5700 & 2.6 & 7.5 & 490 & at $5750 \mathrm{~g} 25 \mathrm{~m} 20 \mathrm{v} 012$ \\
\hline HD $214925^{\mathrm{b}}$. & 224159.4 & -204703 & -2.14 & -2.5 & 3890 & 0.3 & 100.9 & 2320 & at $4000 \mathrm{~g} 05 \mathrm{~m} 20 \mathrm{v} 010$ \\
\hline HD $218732^{\mathrm{b}}$. & 231025.4 & -131834 & -2.00 & -2.8 & 3900 & 0.2 & 112.3 & 1720 & at $4000 \mathrm{~g} 05 \mathrm{~m} 20 \mathrm{v} 012$ \\
\hline 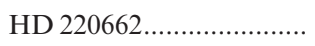 & 232541.6 & -235620 & -1.75 & -1.3 & 4450 & 1.3 & 34.3 & 1810 & at $4500 \mathrm{~g} 15 \mathrm{~m} 15 \mathrm{v} 002$ \\
\hline 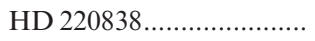 & 232716.5 & -265900 & -1.53 & -1.4 & 4280 & 1.1 & 41.3 & 1420 & at $4250 \mathrm{~g} 10 \mathrm{~m} 15 \mathrm{v} 006$ \\
\hline HD $221170 \ldots \ldots \ldots \ldots \ldots \ldots \ldots$ & 232928.8 & +302557 & -2.26 & -1.7 & 4410 & 1.1 & 42.5 & 690 & at $4500 \mathrm{~g} 10 \mathrm{~m} 20 \mathrm{v} 008$ \\
\hline HD $222434 \ldots \ldots \ldots \ldots \ldots$ & 234041.4 & -344146 & -1.56 & -1.2 & 4430 & 1.3 & 32.9 & 950 & at $4500 \mathrm{~g} 15 \mathrm{~m} 15 \mathrm{v} 004$ \\
\hline
\end{tabular}

Note.--Units of right ascension are hours, minutes, and seconds, and units of declination are degrees, arcminutes, and arcseconds.

${ }^{a}$ Spectroscopic metallicity adopted (see $\left.\S 4.1\right)$.

b Stars requiring $M(V)$ following NBP.

c Stars with special problems (see $\S 4.1$ ).

\subsection{Temperature}

The basis for our temperature estimates is the set of color$T_{\text {eff }}$ relations derived by Alonso, Arribas, \& Martínez Roger (1999) based on infrared flux method determinations. The relations use their own "TCS" system $V-K$ colors, and Johnson $V-R$ and $R-I$ colors, so we had to transform the $R-I$ values of Table 1 into the Johnson system. We employed the relations given by Fernie (1983) to do so. When more than one temperature estimate was available, as was most often the case, we employed straight averages. In the 57 cases where we derived three or more temperature estimates, the mean of the formal standard deviations, $\left\langle\sigma\left(T_{\text {eff }}\right)\right\rangle$, was $45 \mathrm{~K}$.

\subsection{Gravity and Radius}

To select a synthetic spectrum to use as the optimal template for the correlation analysis for each star's observed spectra, we require estimates of the gravities of our program stars. We derive these from the estimated stellar luminosities and temperatures, and hence radii, and an assumed mass of $0.8 M_{\odot}$, appropriate for stars at the main-sequence turnoff in globular clusters. The luminosities are estimated from the $M_{V}$ values after addition of the bolometric correction. Alonso et al. (1999) also provide relations between $\mathrm{BC}_{V}$ and the various color indexes. Table 2 summarizes the resultant atmospheric parameters for our program stars.

Figure 1 plots the derived temperatures and gravities for our program stars versus the 14 Gyr model isochrones of Straniero \& Chieffi (1991). The parallelogram encloses stars

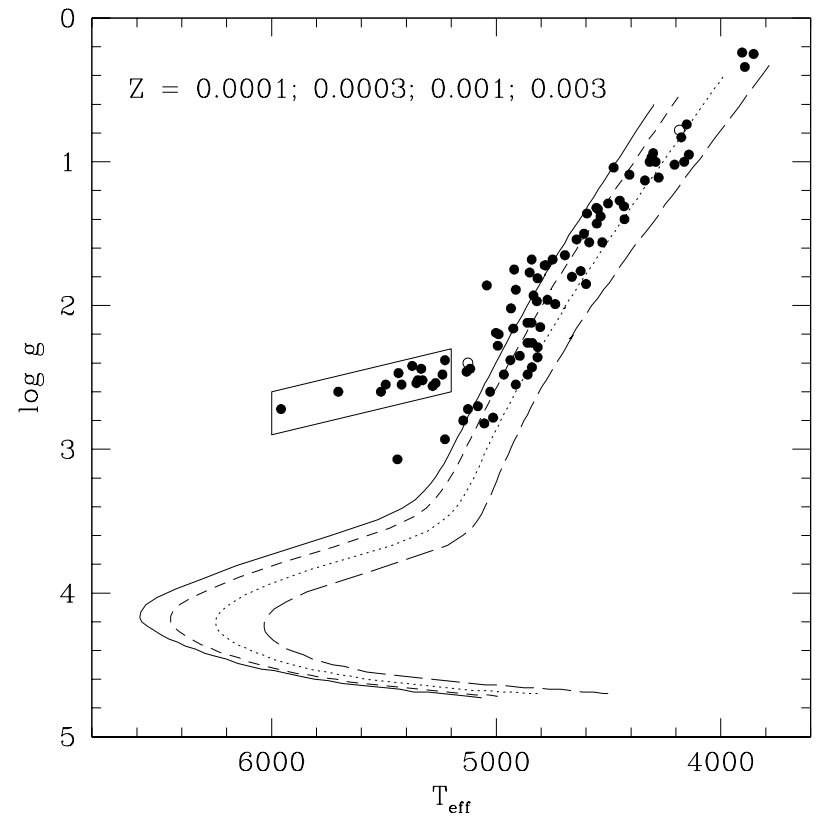

FIG. 1.-Comparison between the gravities and temperatures estimated for our program stars and the 14 Gyr model isochrones of Straniero \& Chieffi (1991). The more metal-poor isochrones lie at higher temperatures. Open circles denote the two $\mathrm{CH}$-strong stars. The parallelogram encloses the RHB stars. 
we consider to be on the RHB, and we have plotted the two $\mathrm{CH}$-strong stars as circles. There is excellent agreement between the data points and the isochrones on the RGB, suggesting that our derivations of the atmospheric parameters are quite plausible.

\subsection{Synthetic Spectrum Templates}

We use these derived atmospheric parameters to select the optimum synthetic spectrum to be employed as the template to derive the radial velocity. These are given in the final column of Table 2. The shorthand is as follows: "a " refers to models computed with line opacities and atmospheric abundances of " $\alpha$ " elements $(\mathrm{O}, \mathrm{Ne}, \mathrm{Mg}, \mathrm{Si}, \mathrm{S}, \mathrm{Ar}, \mathrm{Ca}$, and Ti) increased by 0.4 dex above solar abundance ratios. The " $t$ " precedes the temperature designation, " $g$ " indicates $\log g$, and " $\mathrm{m}$ " refers to "minus" for the metallicity (there being no "p" for "plus" in this sample of metal-poor stars). Thus the first entry, for HD 20, "at5250g25m15," refers to a synthetic spectrum computed with $T_{\text {eff }}=5250 \mathrm{~K}$, $\log g=2.5$, and $[\mathrm{Fe} / \mathrm{H}]=-1.5$ (but with " $\alpha$ " elements enhanced by 0.4 dex).

With temperature, gravity, and metallicity estimates for each star, we then empirically determined the rotational broadening. The average correlation value was computed for all the observed spectra of each star using all available rotationally broadened templates with the adopted values of temperature, gravity, and metallicity. The template with rotational broadening that yielded the highest average correlation was chosen for final use in all the radial velocity measures. These values are indicated by " $\mathrm{v} x x x$ " in the template descriptions in Table 2, where " $x x x$ " is the rotational velocity in kilometers per second. For HD 20, "at $5250 \mathrm{~g} 25 \mathrm{~m} 15 \mathrm{v} 006$ " refers to the model atmosphere and synthetic spectrum defined by the atmospheric parameters described above, with the emergent spectrum broadened by $v_{\text {rot }}=6 \mathrm{~km} \mathrm{~s}^{-1}$ (as well as by the instrumental broadening of $8.5 \mathrm{~km} \mathrm{~s}^{-1}$ ).

Each observed spectrum was compared with the appropriate synthetic spectrum that provided the closest match in temperature, gravity, and metallicity estimated from the photometry. In fact, we ran the correlations of the all the stellar spectra against a very large number of synthetic spectrum templates, and in all cases but one, the average ratio of the peak of the power spectrum to the mean noise level supported our choice. The exception was $\mathrm{BD}+44^{\circ} 493$. With $T_{\text {eff }}=5510 \mathrm{~K}, \log g=2.6$, and $[\mathrm{Fe} / \mathrm{H}]=-2.71$, the appropriate synthetic spectrum, cited in Table 2, was "at5500g25m25v004." However, "at6000g35m30v004" produced a noticeably better set of correlations. The mean radial velocity from the two different templates changed from -150.64 to $-150.74 \mathrm{~km}$ $\mathrm{s}^{-1}$, and the derived $v_{\text {rot }} \sin i$ value changed from 3.9 to $3.1 \mathrm{~km} \mathrm{~s}^{-1}$. To maintain consistency with the other program stars, we choose to report values using the synthetic spectrum template that provides the best estimate of the atmospheric parameters, but the reader is warned that we may have underestimated the star's reddening, and hence its temperature and gravity. It is assumed to be an RHB star in this paper, but it may be a hotter subgiant.

\section{RADIAL VELOCITIES}

Table 3 provides the individual heliocentric radial velocity results derived for each star using the tool RVSAO (Kurtz \& Mink 1998) running inside the IRAF ${ }^{2}$ environment. Table 4 summarizes the results of these measurements obtained for each star. We list the number of observations, the span of the observations in days, the mean radial velocity, and the uncertainty of the mean velocity. Note that for the binary stars, the mean radial velocity is not as appropriate as the systemic velocity that emerges from the orbital solution. For stars with orbital solutions, we therefore list here the systemic velocity and its uncertainty. We also list the measured rms external error $E$ and the mean internal error $I$ of the velocity measurements (see Kurtz \& Mink 1998), and the ratio $E / I$. Large values of $E / I$ are suggestive of radial velocity variability.

Another powerful indicator of radial velocity variability is the probability $P\left(\chi^{2}\right)$ that the $\chi^{2}$ value could be larger than observed because of Gaussian errors for a star that actually has constant velocity. We employ the internal error

${ }^{2}$ IRAF (the Image Reduction and Analysis Facility) is distributed by the National Optical Astronomy Observatory, which is operated by the Association of Universities for Research in Astronomy, Inc., under cooperative agreement with the National Science Foundation.

TABLE 3

Radial Velocities

\begin{tabular}{|c|c|c|c|c|}
\hline Star & Telescope & HJD & $\begin{array}{c}v_{\text {rad }} \\
\left(\mathrm{km} \mathrm{s}^{-1}\right)\end{array}$ & $\begin{array}{c}\sigma \\
\left(\mathrm{km} \mathrm{s}^{-1}\right)\end{array}$ \\
\hline \multirow[t]{10}{*}{ HD $20\left(00^{\mathrm{h}} 05^{\mathrm{m}} 15^{\mathrm{s}} 3,-27^{\circ} 16^{\prime} 18^{\prime \prime}\right) \ldots \ldots \ldots \ldots . . .}$. & M & $2,446,006.67547$ & -57.75 & 0.43 \\
\hline & $\mathrm{T}$ & $2,446,361.77031$ & -56.78 & 0.38 \\
\hline & $\mathrm{T}$ & $2,446,369.79985$ & -56.58 & 0.34 \\
\hline & M & $2,446,393.62169$ & -58.04 & 0.49 \\
\hline & M & $2,446,398.57495$ & -57.44 & 0.36 \\
\hline & M & $2,446,725.71814$ & -57.31 & 0.42 \\
\hline & $\mathrm{T}$ & $2,447,044.86526$ & -56.77 & 0.36 \\
\hline & M & $2,447,345.98195$ & -57.01 & 0.36 \\
\hline & M & $2,447,696.97977$ & -57.25 & 0.35 \\
\hline & $\mathrm{T}$ & $2,448,141.87887$ & -57.03 & 0.42 \\
\hline
\end{tabular}

Note.-Table 3 is presented in its entirety in the electronic editon of the Astronomical Journal. A portion is shown here for guidance regarding its form and content. 
TABLE 4

Radial Velocity Summary

\begin{tabular}{|c|c|c|c|c|c|c|c|c|c|c|}
\hline Star & $N$ & Span & $\left\langle v_{\mathrm{rad}}\right\rangle$ & $\sigma\left(v_{\mathrm{rad}}\right)$ & E & $I$ & $E / I$ & $\chi^{2}$ & $P\left(\chi^{2}\right)$ & $v_{\text {rot }}$ \\
\hline HD $20 \ldots$ & 13 & 4641 & -57.18 & 0.11 & 0.41 & 0.40 & 1.01 & 12.08 & 0.439407 & 5.9 \\
\hline HD 97. & 32 & 6670 & 75.60 & 0.17 & 0.96 & 0.61 & 1.59 & 70.88 & 0.000059 & 4.0 \\
\hline $\mathrm{CD}-23^{\circ} 72$ & 11 & 4963 & 20.67 & 0.24 & 0.71 & 0.80 & 0.89 & 8.08 & 0.621061 & 8.9 \\
\hline HD $3008 \ldots \ldots \ldots \ldots \ldots \ldots \ldots$ & 123 & 6521 & -80.88 & 0.15 & 1.66 & 0.64 & 2.58 & 834.37 & 0.000000 & 9.2 \\
\hline HD $3179 \ldots \ldots \ldots \ldots \ldots \ldots \ldots$ & 15 & 4988 & -74.69 & 0.27 & 1.06 & 0.65 & 1.62 & 32.55 & 0.003349 & 5.2 \\
\hline $\mathrm{CD}-38^{\circ} 245 \ldots \ldots \ldots \ldots \ldots$ & 7 & 4794 & 46.43 & 0.47 & 1.24 & 0.98 & 1.27 & 6.41 & 0.379229 & 3.4 \\
\hline $\mathrm{BD}-11^{\circ} 145 \ldots \ldots$. & 38 & 4470 & -93.34 & 0.12 & 0.74 & 0.68 & 1.09 & 47.54 & 0.114855 & 5.2 \\
\hline HD 5426 . & 11 & 5856 & 27.44 & 0.21 & 0.69 & 0.51 & 1.37 & 15.63 & 0.110821 & 6.3 \\
\hline $\mathrm{BD}-20^{\circ} 170 \ldots \ldots$ & 10 & 4963 & -5.62 & 0.20 & 0.40 & 0.64 & 0.63 & 3.75 & 0.927087 & 5.4 \\
\hline $\mathrm{CD}-30^{\circ} 298 \ldots \ldots \ldots \ldots$ & 16 & 5144 & 27.76 & 0.20 & 0.79 & 0.73 & 1.09 & 12.82 & 0.615945 & 3.5 \\
\hline 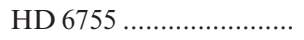 & 34 & 1866 & -318.36 & 0.12 & 6.11 & 0.42 & 14.44 & 6621.62 & $0.003105^{\mathrm{a}}$ & 3.5 \\
\hline HD 9051 . & 9 & 5057 & -72.66 & 0.19 & 0.41 & 0.58 & 0.70 & 3.60 & 0.891379 & 0.5 \\
\hline $\mathrm{BD}-18^{\circ} 271 \ldots \ldots \ldots \ldots \ldots$ & 53 & 3728 & -209.94 & 0.14 & 1.02 & 0.65 & 1.58 & 126.40 & 0.000000 & 7.3 \\
\hline HD 13359 ........................ & 11 & 4988 & 30.45 & 0.25 & 0.68 & 0.82 & 0.83 & 7.08 & 0.718164 & 8.3 \\
\hline $\mathrm{BD}-22^{\circ} 395 \ldots \ldots$. & 19 & 5049 & 102.54 & 0.22 & 0.97 & 0.63 & 1.52 & 37.11 & 0.005076 & 6.5 \\
\hline $\mathrm{BD}+44^{\circ} 493 \ldots \ldots \ldots \ldots \ldots \ldots$ & 28 & 4982 & -150.64 & 0.15 & 0.81 & 0.73 & 1.11 & 31.91 & 0.235263 & 3.9 \\
\hline $\mathrm{BD}-10^{\circ} 548 \ldots \ldots \ldots \ldots \ldots$ & 14 & 4790 & 237.77 & 0.19 & 0.71 & 0.68 & 1.05 & 12.93 & 0.453086 & 3.0 \\
\hline $\mathrm{CD}-30^{\circ} 1121 \ldots \ldots$ & 8 & 5100 & 104.75 & 0.19 & 0.55 & 0.53 & 1.03 & 9.18 & 0.239657 & 2.5 \\
\hline HD $21581 \ldots \ldots \ldots . . .$. & 11 & 4708 & 153.23 & 0.22 & 0.75 & 0.61 & 1.22 & 17.25 & 0.068995 & 5.7 \\
\hline HD 23798 .. & 10 & 4459 & 89.43 & 0.21 & 0.66 & 0.39 & 1.67 & 24.62 & 0.003422 & 5.0 \\
\hline HD $27928 \ldots$. & 32 & 5819 & 44.65 & 0.21 & 2.70 & 0.43 & 6.26 & 1156.90 & $0.116023^{\mathrm{a}}$ & 1.7 \\
\hline HD $36702 \ldots \ldots \ldots \ldots \ldots \ldots$ & 11 & 4805 & 122.71 & 0.20 & 0.65 & 0.40 & 1.62 & 28.27 & 0.001637 & 6.5 \\
\hline $\mathrm{BD}-19^{\circ} 1422 \ldots \ldots \ldots \ldots \ldots$ & 19 & 6142 & 56.79 & 0.16 & 0.70 & 0.66 & 1.06 & 21.99 & 0.232388 & 5.6 \\
\hline HD $45282 \ldots \ldots \ldots \ldots \ldots \ldots$ & 11 & 5021 & 306.03 & 0.19 & 0.64 & 0.56 & 1.14 & 12.28 & 0.266690 & 5.0 \\
\hline HD $63791 \ldots \ldots \ldots \ldots \ldots \ldots$ & 23 & 4885 & -108.44 & 0.10 & 0.34 & 0.47 & 0.72 & 13.72 & 0.911187 & 3.7 \\
\hline 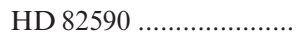 & 14 & 4863 & 214.40 & 0.25 & 0.78 & 0.95 & 0.82 & 13.41 & 0.416634 & 13.0 \\
\hline 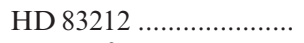 & 11 & 5096 & 109.13 & 0.16 & 0.47 & 0.52 & 0.89 & 10.78 & 0.375289 & 7.3 \\
\hline $\mathrm{BD}+30^{\circ} 2034 \ldots \ldots \ldots \ldots \ldots$ & 25 & 4016 & 93.91 & 0.16 & 12.68 & 0.62 & 20.33 & 12595.77 & $0.080037^{\mathrm{a}}$ & 8.9 \\
\hline $\mathrm{CD}-30^{\circ} 8626 \ldots \ldots \ldots \ldots \ldots$ & 10 & 5496 & 265.94 & 0.13 & 0.28 & 0.41 & 0.69 & 4.84 & 0.847621 & 5.0 \\
\hline HD $93529 \ldots \ldots \ldots$ & 11 & 5146 & 146.26 & 0.18 & 0.61 & 0.50 & 1.22 & 17.64 & 0.061401 & 2.7 \\
\hline $\mathrm{BD}+22^{\circ} 2411 .$. & 153 & 5501 & 34.58 & 0.09 & 1.07 & 0.54 & 1.98 & 593.48 & 0.000000 & 7.3 \\
\hline $\mathrm{BD}-1^{\circ} 2582 \ldots$ & 37 & 6562 & -0.14 & 0.20 & 1.20 & 0.70 & 1.72 & 118.45 & $0.203999^{\mathrm{a}}$ & 6.9 \\
\hline HD $103545 \ldots .$. & 12 & 5110 & 179.34 & 0.17 & 0.53 & 0.60 & 0.88 & 9.06 & 0.616515 & 5.0 \\
\hline $\mathrm{BD}+9^{\circ} 2574 \ldots \ldots \ldots \ldots \ldots$ & 10 & 5113 & -48.87 & 0.20 & 0.59 & 0.64 & 0.92 & 6.65 & 0.673832 & 2.5 \\
\hline HD $107752 \ldots \ldots \ldots \ldots \ldots \ldots$ & 14 & 5146 & 219.77 & 0.20 & 0.75 & 0.62 & 1.21 & 20.60 & 0.081311 & 4.6 \\
\hline HD $108317 \ldots \ldots \ldots \ldots \ldots \ldots$ & 42 & 3432 & 7.45 & 0.15 & 0.95 & 0.57 & 1.66 & 114.56 & $0.006152^{\mathrm{a}}$ & 5.1 \\
\hline HD $108577 \ldots \ldots \ldots \ldots \ldots \ldots$ & 15 & 5142 & -111.31 & 0.17 & 0.62 & 0.65 & 0.96 & 12.22 & 0.588413 & 6.9 \\
\hline 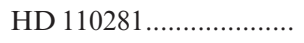 & 40 & 4149 & 141.87 & 0.23 & 1.45 & 0.64 & 2.27 & 214.73 & 0.000000 & 11.5 \\
\hline 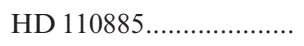 & 10 & 5109 & -47.89 & 0.24 & 0.76 & 0.75 & 1.01 & 8.29 & 0.504899 & 8.2 \\
\hline HD $111721 \ldots \ldots \ldots \ldots \ldots \ldots$ & 13 & 4180 & 21.12 & 0.16 & 0.46 & 0.57 & 0.80 & 6.97 & 0.859752 & 3.0 \\
\hline $\mathrm{BD}+10^{\circ} 2495 .$. & 40 & 1488 & 258.30 & 0.12 & 8.87 & 0.61 & 14.49 & 8499.13 & $0.516997^{\mathrm{a}}$ & 3.8 \\
\hline $\mathrm{BD}+12^{\circ} 2547 \ldots \ldots \ldots \ldots \ldots$ & 10 & 5167 & 5.76 & 0.19 & 0.48 & 0.59 & 0.81 & 5.61 & 0.778480 & 3.9 \\
\hline $\mathrm{BD}+3^{\circ} 2782 \ldots \ldots \ldots \ldots \ldots$ & 12 & 5122 & 31.96 & 0.19 & 0.64 & 0.52 & 1.24 & 16.53 & 0.122496 & 5.3 \\
\hline HD $118055 \ldots .$. & 10 & 5130 & -100.73 & 0.17 & 0.53 & 0.54 & 0.97 & 9.18 & 0.420647 & 7.4 \\
\hline $\mathrm{BD}+18^{\circ} 2757 \ldots \ldots \ldots \ldots \ldots$ & 28 & 1479 & -23.64 & 0.14 & 5.92 & 0.66 & 9.02 & 2280.15 & $0.164932^{\mathrm{a}}$ & 7.8 \\
\hline 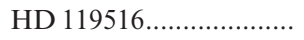 & 12 & 4914 & -285.95 & 0.21 & 0.71 & 0.71 & 1.00 & 10.99 & 0.444472 & 9.1 \\
\hline $\mathrm{CD}-33^{\circ} 9314 \ldots \ldots \ldots \ldots$ & 12 & 5141 & 407.36 & 0.13 & 0.40 & 0.46 & 0.86 & 7.83 & 0.728741 & 5.0 \\
\hline HD $121135 \ldots \ldots \ldots \ldots \ldots \ldots$ & 11 & 5149 & 125.76 & 0.18 & 0.60 & 0.59 & 1.00 & 10.68 & 0.382661 & 8.6 \\
\hline HD $121261 \ldots \ldots \ldots \ldots \ldots \ldots$ & 12 & 5142 & 99.90 & 0.26 & 0.90 & 0.46 & 1.97 & 51.12 & 0.000000 & 8.0 \\
\hline 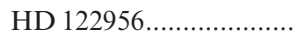 & 12 & 5073 & 165.69 & 0.14 & 0.48 & 0.43 & 1.11 & 12.74 & 0.310712 & 6.3 \\
\hline $\mathrm{BD}+9^{\circ} 2860 \ldots \ldots \ldots \ldots \ldots$ & 11 & 5131 & -19.33 & 0.20 & 0.54 & 0.67 & 0.81 & 7.54 & 0.673934 & 3.9 \\
\hline 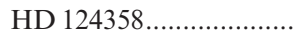 & 13 & 5126 & 324.83 & 0.20 & 0.72 & 0.65 & 1.11 & 15.31 & 0.224798 & 6.7 \\
\hline $\mathrm{BD}+9^{\circ} 2870 \ldots$ & 16 & 5074 & -120.26 & 0.18 & 0.74 & 0.61 & 1.20 & 26.60 & 0.032150 & 5.2 \\
\hline $\mathrm{BD}+8^{\circ} 2856 \ldots \ldots \ldots \ldots \ldots$ & 15 & 5116 & 64.51 & 0.21 & 0.81 & 0.56 & 1.46 & 31.80 & 0.004281 & 8.9 \\
\hline 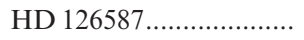 & 10 & 5112 & 149.40 & 0.17 & 0.39 & 0.55 & 0.71 & 4.69 & 0.860681 & 4.3 \\
\hline $\mathrm{BD}+18^{\circ} 2890 .$. & 25 & 1046 & -26.65 & 0.10 & 6.41 & 0.59 & 10.90 & 3297.89 & $0.899407^{\mathrm{a}}$ & 5.1 \\
\hline $\mathrm{BD}-8^{\circ} 3901 \ldots \ldots \ldots \ldots \ldots$ & 10 & 5126 & -108.63 & 0.21 & 0.66 & 0.57 & 1.17 & 14.62 & 0.101854 & 4.3 \\
\hline $\mathrm{BD}+18^{\circ} 2976 \ldots \ldots \ldots \ldots \ldots$ & 103 & 5368 & -167.97 & 0.09 & 3.59 & 0.55 & 6.53 & 4910.94 & $0.001932^{\mathrm{a}}$ & 5.2 \\
\hline 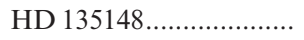 & 33 & 1953 & -90.86 & 0.15 & 3.48 & 0.56 & 6.21 & 1491.26 & $0.003770^{\mathrm{a}}$ & 7.8 \\
\hline $\mathrm{BD}+1^{\circ} 3070 \ldots \ldots \ldots \ldots \ldots$ & 41 & 3333 & -330.11 & 0.11 & 2.92 & 0.67 & 4.38 & 912.52 & $0.541643^{\mathrm{a}}$ & 5.0 \\
\hline HD $141531 \ldots \ldots \ldots \ldots \ldots \ldots$ & 12 & 5140 & 2.13 & 0.17 & 0.52 & 0.57 & 0.91 & 10.75 & 0.464533 & 7.5 \\
\hline $\mathrm{BD}+5^{\circ} 3098 \ldots \ldots \ldots \ldots \ldots$ & 25 & 4900 & -160.70 & 0.16 & 0.79 & 0.73 & 1.08 & 28.49 & 0.239878 & 5.2 \\
\hline $\mathrm{BD}+11^{\circ} 2998 \ldots \ldots \ldots \ldots \ldots$ & 34 & 5068 & 50.11 & 0.10 & 0.51 & 0.58 & 0.88 & 28.07 & 0.711334 & 6.8 \\
\hline $\mathrm{BD}+9^{\circ} 3223 \ldots \ldots \ldots \ldots \ldots$ & 24 & 5016 & 67.11 & 0.14 & 0.60 & 0.67 & 0.89 & 20.42 & 0.616212 & 4.8 \\
\hline $\mathrm{BD}+13^{\circ} 3683 \ldots \ldots \ldots \ldots$ & 35 & 1533 & 92.54 & 0.17 & 16.98 & 0.94 & 17.97 & 12482.67 & $0.589375^{\mathrm{a}}$ & 5.1 \\
\hline
\end{tabular}


TABLE 4-Continued

\begin{tabular}{|c|c|c|c|c|c|c|c|c|c|c|}
\hline Star & $N$ & Span & $\left\langle v_{\mathrm{rad}}\right\rangle$ & $\sigma\left(v_{\mathrm{rad}}\right)$ & E & $I$ & $E / I$ & $\chi^{2}$ & $P\left(\chi^{2}\right)$ & $v_{\text {rot }}$ \\
\hline HD $171496 \ldots .$. & 13 & 4936 & -39.34 & 0.14 & 0.52 & 0.48 & 1.09 & 16.58 & 0.166112 & 7.1 \\
\hline HD $175305 \ldots \ldots \ldots \ldots \ldots \ldots$ & 10 & 2956 & -184.86 & 0.19 & 0.59 & 0.38 & 1.54 & 21.66 & 0.010031 & 1.5 \\
\hline HD $176203 \ldots \ldots \ldots$. & 12 & 5074 & 68.73 & 0.18 & 0.49 & 0.64 & 0.78 & 9.98 & 0.531835 & 6.8 \\
\hline HD $181007 \ldots$ & 12 & 5110 & -2.31 & 0.19 & 0.56 & 0.67 & 0.84 & 8.49 & 0.668407 & 4.2 \\
\hline HD $184266 \ldots \ldots$. & 13 & 5110 & -348.86 & 0.19 & 0.70 & 0.70 & 0.99 & 10.61 & 0.562779 & 11.7 \\
\hline 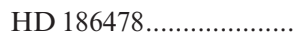 & 29 & 2140 & 30.43 & 0.12 & 2.11 & 0.60 & 3.52 & 422.58 & $0.539690^{\mathrm{a}}$ & 5.3 \\
\hline $\mathrm{BD}-18^{\circ} 5550 \ldots \ldots \ldots \ldots \ldots$ & 12 & 4792 & -125.48 & 0.16 & 0.30 & 0.56 & 0.54 & 3.88 & 0.973291 & 3.9 \\
\hline HD $193242 \ldots \ldots \ldots \ldots \ldots \ldots$ & 14 & 5153 & -128.40 & 0.17 & 0.61 & 0.62 & 0.99 & 14.56 & 0.335743 & 5.5 \\
\hline 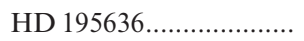 & 43 & 5086 & -258.50 & 0.22 & 1.43 & 1.23 & 1.17 & 39.76 & 0.569690 & 21.5 \\
\hline $\mathrm{BD}-17^{\circ} 6036 \ldots \ldots \ldots \ldots \ldots$ & 12 & 4729 & 19.26 & 0.20 & 0.69 & 0.64 & 1.07 & 14.27 & 0.218387 & 3.9 \\
\hline $\mathrm{BD}-20^{\circ} 6008 \ldots \ldots \ldots \ldots \ldots$ & 12 & 4792 & 22.28 & 0.23 & 0.80 & 0.61 & 1.30 & 23.10 & 0.017100 & 0.5 \\
\hline $\mathrm{BD}-15^{\circ} 5781 \ldots \ldots \ldots \ldots \ldots$ & 15 & 4767 & -76.01 & 0.21 & 0.80 & 0.79 & 1.00 & 12.58 & 0.559899 & 3.4 \\
\hline $\mathrm{BD}-14^{\circ} 5890 \ldots \ldots \ldots \ldots \ldots$ & 13 & 4754 & 117.86 & 0.19 & 0.67 & 0.63 & 1.06 & 15.11 & 0.235724 & 2.2 \\
\hline $\mathrm{CD}-37^{\circ} 14010 \ldots \ldots \ldots \ldots$ & 23 & 1479 & -199.03 & 0.15 & 6.37 & 0.92 & 6.91 & 1033.54 & $0.945032^{\mathrm{a}}$ & 19.4 \\
\hline $\mathrm{BD}-3^{\circ} 5215 \ldots \ldots \ldots \ldots \ldots \ldots$ & 12 & 4740 & -293.73 & 0.23 & 0.64 & 0.78 & 0.82 & 7.19 & 0.783260 & 7.3 \\
\hline 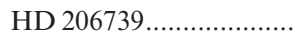 & 16 & 5154 & -57.65 & 0.15 & 0.59 & 0.57 & 1.04 & 16.06 & 0.377992 & 5.1 \\
\hline $\mathrm{BD}-9^{\circ} 5831 \ldots \ldots \ldots \ldots \ldots$ & 12 & 4793 & 14.72 & 0.17 & 0.52 & 0.59 & 0.88 & 9.07 & 0.615630 & 5.1 \\
\hline 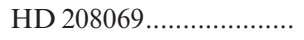 & 9 & 5086 & -166.93 & 0.25 & 0.75 & 0.49 & 1.53 & 20.32 & 0.009189 & 3.7 \\
\hline 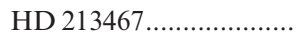 & 11 & 5084 & -72.36 & 0.22 & 0.73 & 0.45 & 1.60 & 32.98 & 0.000275 & 1.5 \\
\hline HD $214362 \ldots \ldots \ldots \ldots \ldots \ldots$ & 13 & 4764 & -92.18 & 0.23 & 0.52 & 0.85 & 0.61 & 4.30 & 0.977329 & 11.1 \\
\hline 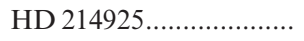 & 36 & 4808 & -327.97 & 0.21 & 1.25 & 0.55 & 2.26 & 207.19 & 0.000000 & 9.7 \\
\hline HD $218732 \ldots \ldots \ldots \ldots \ldots \ldots$ & 46 & 2967 & -312.20 & 0.15 & 1.98 & 0.45 & 4.40 & 981.17 & $0.000000^{\mathrm{a}}$ & 11.1 \\
\hline 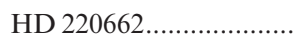 & 11 & 5052 & -76.72 & 0.25 & 0.84 & 0.61 & 1.37 & 17.30 & 0.067990 & 3.0 \\
\hline HD $220838 \ldots \ldots \ldots \ldots \ldots \ldots$ & 10 & 5108 & -22.96 & 0.16 & 0.49 & 0.42 & 1.19 & 14.73 & 0.098586 & 6.5 \\
\hline 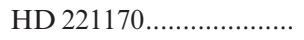 & 18 & 5145 & -121.77 & 0.14 & 0.61 & 0.44 & 1.38 & 29.00 & 0.034570 & 7.4 \\
\hline HD $222434 \ldots \ldots \ldots \ldots \ldots \ldots$ & 28 & 1519 & 8.81 & 0.14 & 7.09 & 0.38 & 18.56 & 9520.71 & $0.001985^{\mathrm{a}}$ & 5.0 \\
\hline
\end{tabular}

a Probability computed using velocity residuals from the orbital solution.

estimate $\sigma_{i, \text { int }}$ for each of $n$ exposures when calculating $\chi^{2}$ :

$$
\chi^{2}=\sum_{i=1}^{n}\left(\frac{x_{i}-\langle x\rangle}{\sigma_{i, \text { int }}}\right)^{2} .
$$

The values of $\sigma_{i, \text { int }}$ are computed by RVSAO. If these internal error estimates are correct and the actual errors are Gaussian, then the distribution of $P\left(\chi^{2}\right)$ should be constant, except for the velocity variables occupying the region near zero probability. To achieve a flat distribution of $P\left(\chi^{2}\right)$ values for $P\left(\chi^{2}\right)>0.05$, we found it necessary to add in quadrature a "floor error" of $0.25 \mathrm{~km} \mathrm{~s}^{-1}$ to each internal error estimate. This floor error compensates for various systematic errors, such as shortcomings in the run-to-run zeropoint corrections, which are not included in the internal error estimates. The $P\left(\chi^{2}\right)$ values are listed in Table 4 . All stars that we have found to be binaries had $P\left(\chi^{2}\right)<10^{-6}$. For those stars, we have replaced $\langle x\rangle$ in equation (1) with the velocity obtained from the orbital solution (or preliminary one, in the cases of $\mathrm{BD}-1^{\circ} 2582$ and HD 108317). In Table 4, we note the resultant $P\left(\chi^{2}\right)$ values calculated using the velocity residuals from the orbital solution.

Figure 2 shows the distributions of $P\left(\chi^{2}\right)$. The hatched regions cover all 16 spectroscopic binary stars. Here we have relied on the values calculated before we took orbital motion into account, and thus the figures reveal all signs of stellar velocity variability. Figure $2 a$ shows that the expected flat distribution is seen for $P\left(\chi^{2}\right)>0.05$, which suggests our estimation of $P\left(\chi^{2}\right)$ is reliable, on average. In Figures $2 b$ and $2 c$, we focus on the distribution of the smaller $P\left(\chi^{2}\right)$ values. Note that few, if any, of our 91 stars should have $P\left(\chi^{2}\right)<0.005$, if they have a constant velocity, yet we find 24 such stars, and 30 with $P\left(\chi^{2}\right)<0.01$. Thus, many of our program stars show varying degrees of radial velocity variations, which may be due to orbital motion, radial oscillations, nonperiodic atmospheric motions, starspots, or occasional problems with our velocity measurements. Regarding this last point, if our internal error estimates are
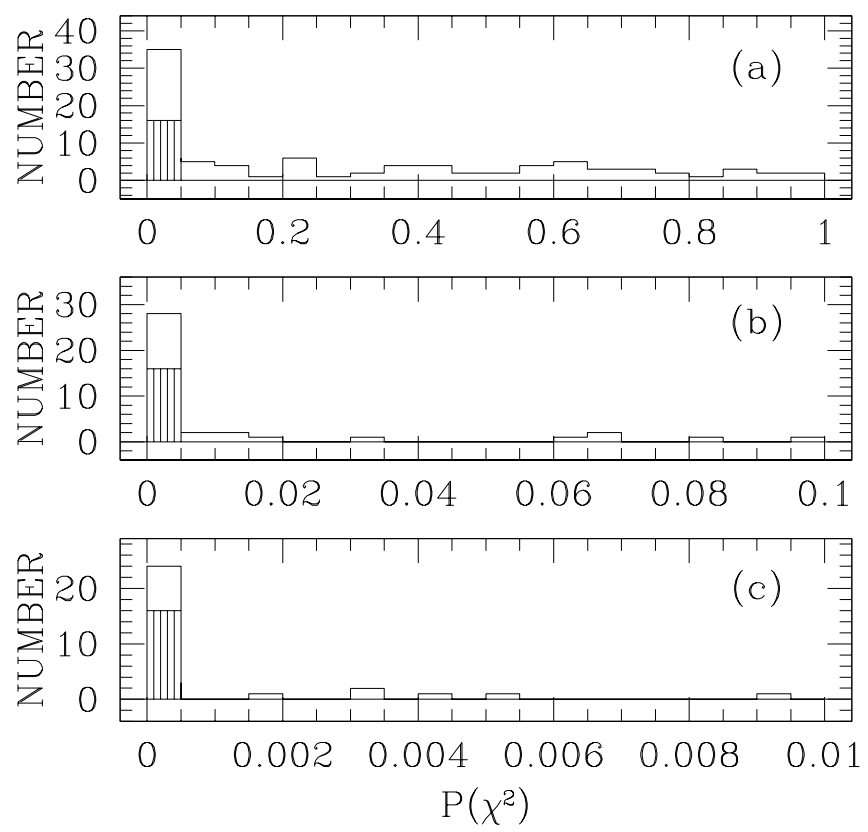

FIG. 2.-Distribution of probabilities of $\chi^{2}$ for our program stars. There is an excess of small values, indicating a substantial number of stars with variable velocities. Vertical hatching indicates stars found to be spectroscopic binaries. 
systematically too optimistic, or there is some source of systematic error that those estimates do not include, or if there are non-Gaussian outliers among the data, we may overestimate $\chi^{2}$ and, hence, underestimate $P\left(\chi^{2}\right)$ in individual cases. We discuss the options for true velocity variations under two broad headings: orbital motion and velocity jitter.

\section{SPECTROSCOPIC BINARIES}

\subsection{Orbital Solutions}

To identify candidate spectroscopic binaries, we relied heavily on the $P\left(\chi^{2}\right)$ values, giving special scrutiny to systems with values less than about 0.1 . We also inspected plots of the velocity history for each star, to look for lowamplitude variations such as slow drifts or correlated patterns. In addition, we inspected plots of the power spectrum of the velocities for each star, to look for candidate periodicities. Peaks in the power spectrum more than about 7 times stronger than the average noise often led to convincing solutions. For all the stars suspected to have excess velocity variation but with no obvious periodicity in the power spectrum, we subjected the data to a brute-force analysis by trying to fit orbits at thousands of trial periods between 0.5 and several thousand days.

Even after promising orbital solutions emerged from the analysis outlined above, we continued to observe the candidate binaries, often doubling the total number of observations before we were satisfied that the solution was ready for publication. For this analysis we used codes developed at the Center for Astrophysics by G. Torres, based on procedures and codes developed by T. Mazeh at Tel Aviv University. Our orbital solutions are summarized in Table 5, where we give the star name, followed by the orbital period, the systemic velocity, the radial velocity amplitude, the orbital eccentricity, the longitude of the ascending node, the time of passage of the ascending node, $a_{1} \sin i$, and the mass function. With each of these values, we provide the standard error. Columns (10)-(11) contain the number of orbital cycles covered by the data and the standard error, $\sigma$, of the "observed minus calculated," $O-C$, velocity residuals. Columns (12)-(13) include the number of observations $N$ and the span of the observations. The mass function $f(M)$ is defined by

$$
f(M)=\sin ^{3} i \frac{M_{2}^{3}}{\left(M_{1}+M_{2}\right)^{2}} .
$$

The velocity curves for the 14 orbital solutions and the individual observed velocities are plotted in Figure 3.

Figure 4 shows the velocity histories of $\mathrm{BD}-1^{\circ} 2582$ and HD 108317. The velocity histories and the extremely low values of $P\left(\chi^{2}\right)\left(<10^{-6}\right)$ indicate that both are also spectroscopic binary systems, but we have not yet covered full orbital cycles, despite 18.0 and $9.4 \mathrm{yr}$ of coverage, respectively. Final orbital solutions will require further observations.

One of our program stars, BD $+13^{\circ} 3683$, had already been recognized as a spectroscopic binary, and a solution obtained, by Jasniewicz \& Mayor (1986). We have reported their solution in Table 5 for comparison: the agreement with our orbital solution is excellent. Jasniewicz \& Mayor (1988) also noted that HD 6755 is almost certainly a spectroscopic binary and that their preliminary orbital solution suggested a period of about 1650 days. This agrees extremely well with our derived period of $1641 \pm 24$ days.

Our radial velocity program does not include all the metal-poor red giants identified by ATT or NBP, so we conducted a search through the SIMBAD database to see whether other spectroscopic binary orbits had been obtained for stars not in our program. Our search resulted in two successes: HD 89499 (Ardeberg \& Lindgren 1991) and HD 170737 (Griffin 1980). We have included the orbital solutions for these stars in Table 5 for completeness. Unfortunately, the mass function uncertainty, the longitude of the ascending node, the goodness of fit, and the individual radial velocity data (from which the number of orbital cycles covered by the data may be determined) have not been published for HD 89499.

\subsection{Spectroscopic Binary Frequency}

Short-period binary systems are expected to undergo mass transfer and possibly merge as the primary evolves into the red giant stage. While the frequency of such mergers is probably small, given the observed period distribution of metal-poor dwarfs, it is nonetheless worth comparing the derived spectroscopic binary frequencies of metal-poor dwarfs and giants to see if such an effect is large enough to be detected.

The original selection of red giants was intended to include only those stars with $[\mathrm{Fe} / \mathrm{H}] \leq-1.4$, but as Table 2 reveals, some stars proved to be somewhat more metal-rich than we expected. There are 16 spectroscopic binaries in our sample (including $\mathrm{BD}-1^{\circ} 2582$ and HD 108317), and the spectroscopic binary fraction is $18 \% \pm 4 \%$ if we employ the full sample, or $19 \% \pm 5 \%$ if we restrict the sample to the 85 stars with $[\mathrm{Fe} / \mathrm{H}] \leq-1.4$. This drops to $16 \% \pm 4 \%$ if we include only those 14 systems with orbital solutions and periods of less than 6000 days (roughly our longest measured orbital period). For the corresponding binary frequency among the metal-poor field dwarfs, we rely on our studies of stars with high proper motions. Specifically, we employ the database of metallicities and other parameters given in Carney et al. (1994; many of these results have been updated using new data and new analysis tools). Using the orbital solutions published in Latham et al. (2002) and Goldberg et al. (2002), and after excluding stars with uncertain metallicities, as well as post-main-sequence stars, there are 49 dwarfs with $[\mathrm{Fe} / \mathrm{H}] \leq-1.4$ with single-lined or double-lined orbital solutions, plus three more stars that are certainly spectroscopic binaries but whose solutions are not complete. The sample includes 233 stars that appear to have constant velocities, so the binary frequency for the metalpoor dwarfs is $52 /(52+233)=18 \% \pm 3 \%$ if we include all spectroscopic binaries, and $47 /(47+233)=17 \% \pm 2 \%$ if the sample is restricted to only those stars with orbital solutions and periods of less than 6000 days. If we include field blue stragglers (Carney et al. 2001), which ultimately evolve into halo red giants, three more binaries with $[\mathrm{Fe} / \mathrm{H}] \leq$ -1.4 are added, and the two binary frequencies for metalpoor field dwarfs become $19 \% \pm 3 \%$ for all periods and $18 \% \pm 4 \%$ for periods less than 6000 days. We conclude that the binary frequencies of metal-poor field dwarfs and giants are indistinguishable. There is no evidence from the binary frequencies, therefore, of a significant loss of binary systems as the metal-poor dwarfs evolve into red giants. 


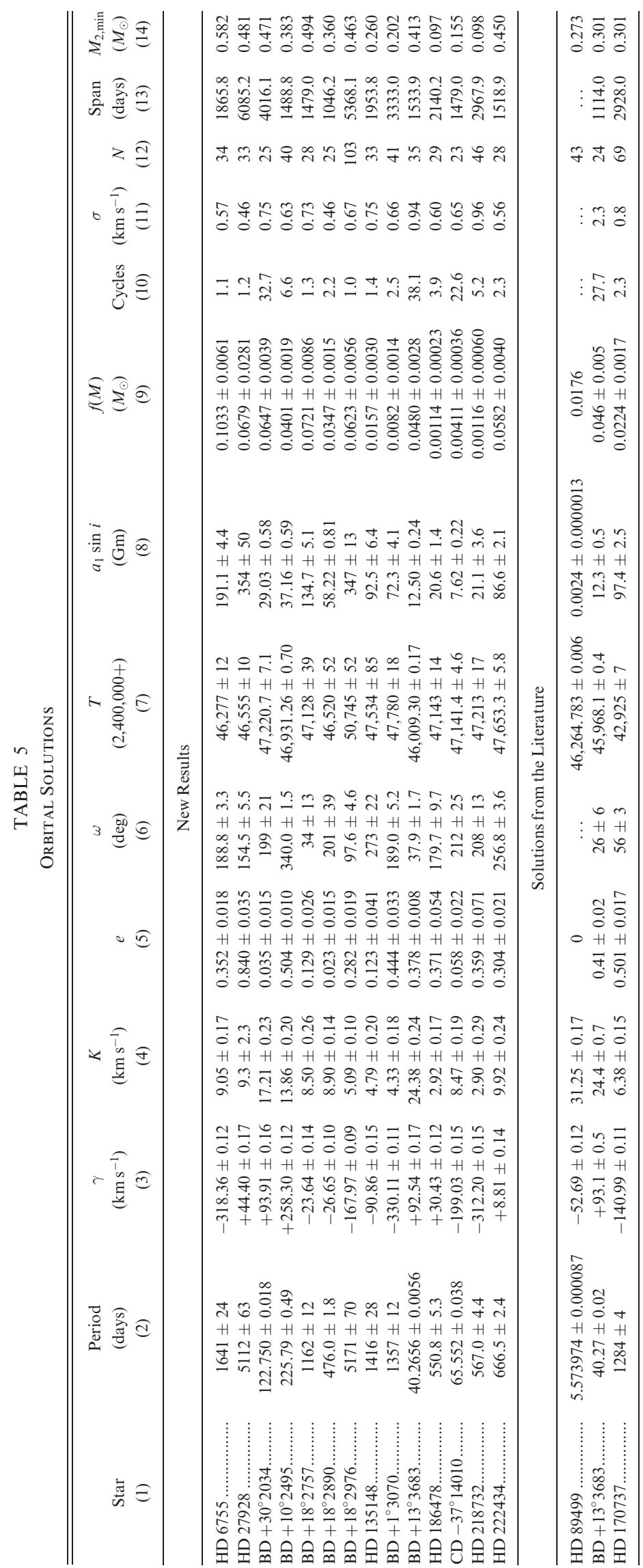



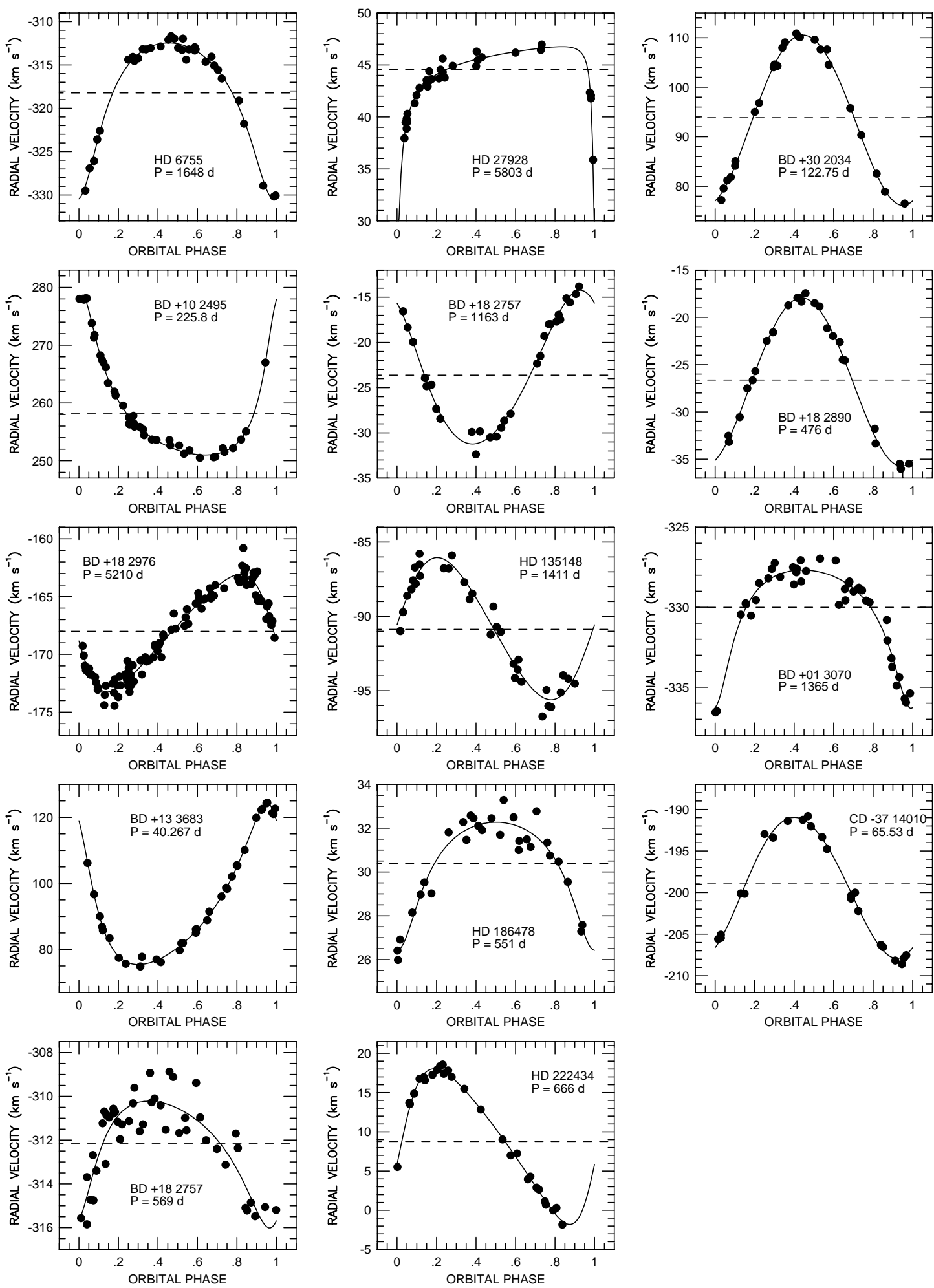

FIG. 3.-Spectroscopic binary orbital solutions for the stars of Table 5

Such mergers do happen, however. In our studies, we have found a few stars with very short orbital periods, from half a day to a few days. These will lead to mergers, but we have not included these stars in the discussion here, because we are unable to determine the metallicities of the stars.

\subsection{Orbital Characteristics}

Possible differences in the binary orbital characteristics for metal-poor field red giants versus dwarfs are worthy of investigation as well. For example, orbital evolution can circularize and lengthen the period of a binary as it undergoes 


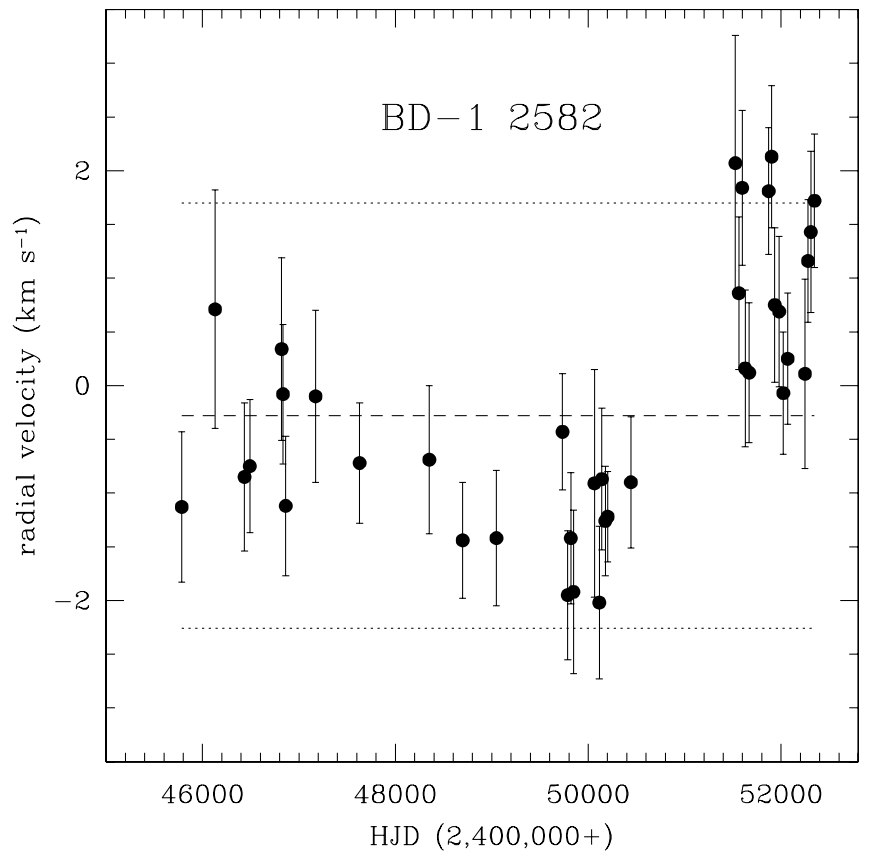

FIG. $4 a$

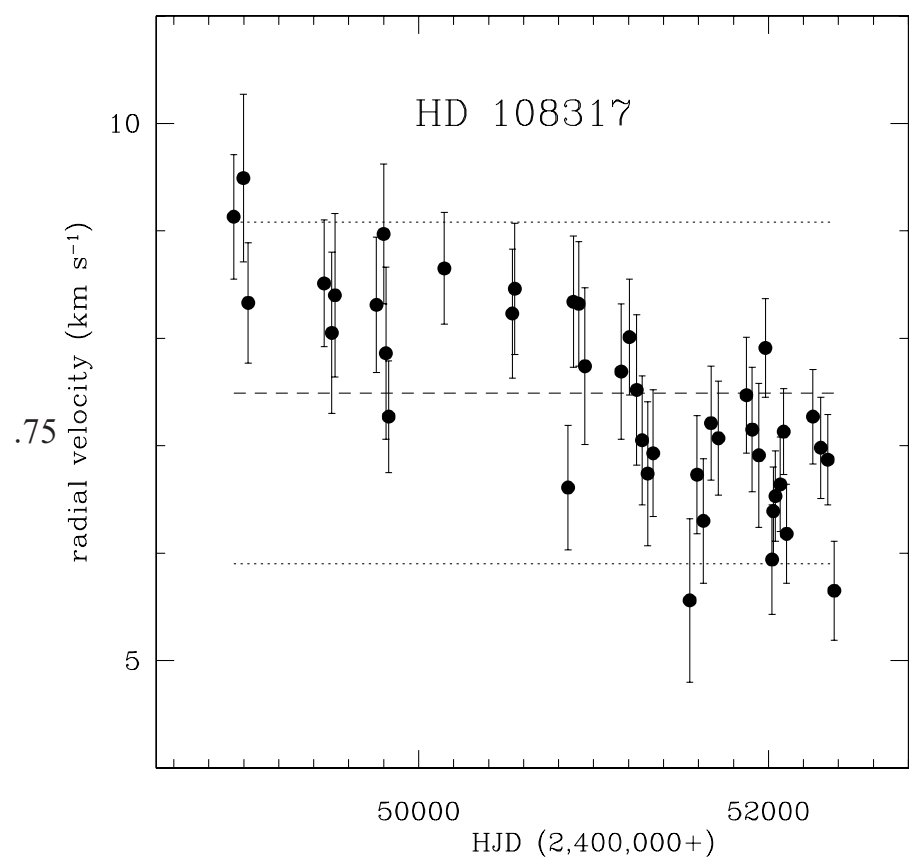

FIG. $4 b$

FIG. 4.-Velocity histories of two binary stars whose orbital solutions are as yet incomplete: $(a) \mathrm{BD}-1^{\circ} 2582$ and $(b)$ HD 108317 . The mean velocities, as well as $\pm 3 \sigma_{\text {int }}$, are shown as horizontal lines.

stable mass transfer. If the binary is neither disrupted nor merged, then the total binary frequency stays the same, but the period and eccentricity distributions change.

In previous papers (Latham et al. 1988, 1992, 2002; Goldberg et al. 2002; Carney et al. 2001), we have drawn attention to the importance of orbital eccentricity as an indicator of possible orbital evolution. For most main-sequence binaries with periods longer than about 10 days, the orbits are found to cover a wide range of eccentricities with a median value of about 0.4 (see, e.g., Duquennoy \& Mayor 1991; Latham et al. 2002). At shorter periods, circular orbits are observed to dominate, presumably as a result of tidal circularization. The transition from eccentric to circular orbits for a population of coeval binaries can be interpreted as a "chronometer" (e.g., Mathieu et al. 1992), because tidal circularization can reach to larger separations and therefore longer periods as the population ages. For the Hyades, with an age of about $500 \mathrm{Myr}$, the transition period is 8.5 days. For M67, with an age of about $5 \mathrm{Gyr}$, the transition period is about 12.4 days. For the oldest stars in the Galaxy, the transition is about 20 days. This is shown in Figure 5, where we have plotted orbital eccentricity versus the logarithm of the period for the population of 47 metal-poor field dwarf spectroscopic binaries with $[\mathrm{M} / \mathrm{H}]<-1.4$ taken from Latham et al. (2002) and Goldberg et al. (2002), along with all the red giant binaries reported in Table 5 .

An initial consideration of Figure 5 reveals that all the short-period $(P<20$ days) binary systems do indeed have nearly circular orbits, including the red giant HD 89499 studied by Ardeberg \& Lindgren (1991). What may not be obvious in a casual inspection of Figure 5, however, is that the dwarfs and giants have somewhat different period and eccentricity distributions. In Figure 6, we compare the cumulative binary fractions for the field dwarfs and giants with $M_{V} \leq-1.4$. It appears that the giants are somewhat deficient in short-period systems. Because of the small sample sizes, it is hard to be definitive, but the KolmogorovSmirnov (K-S) test says that there is an $85 \%$ chance that the two period distributions were drawn from different parent distributions. This suggests that some of the red giants have experienced orbital evolution that has lengthened their periods. This argument is supported by the eccentricity dis-

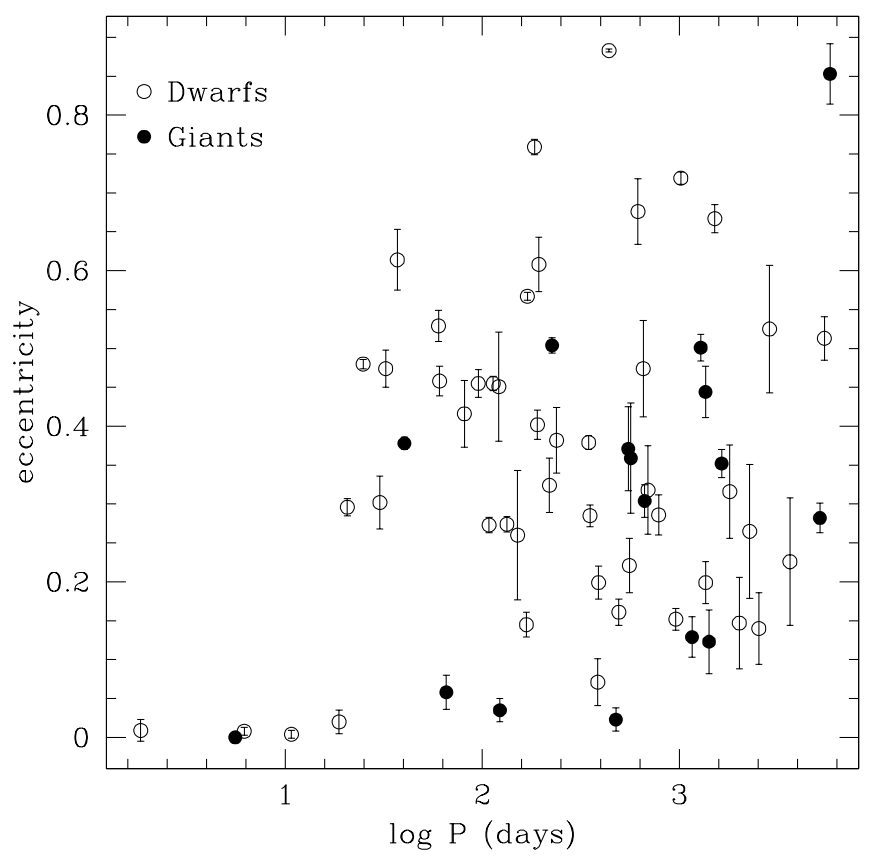

FIG. 5.-Comparison of the orbital periods and eccentricities of field dwarfs and red giants with $[\mathrm{Fe} / \mathrm{H}] \leq-1.4$, with data taken from Table 5 and Latham et al. (2002) and Goldberg et al. (2002). 


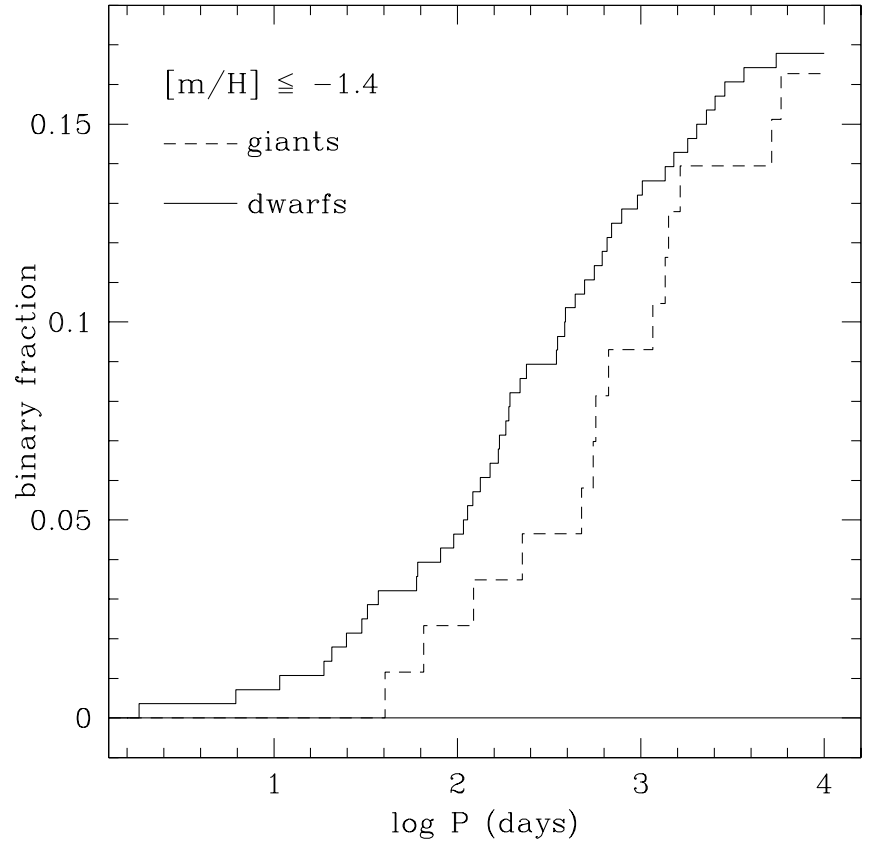

FIG. 6.-Comparison of the cumulative binary frequencies as a function of orbital period of 44 metal-poor field dwarfs (Latham et al. 2002; Goldberg et al. 2002) and the 14 metal-poor field red giant binaries reported in this paper.

tributions. Figure 5 shows that the giants have a significant number of binary systems with small orbital eccentricities. If we consider only the 11 giants with $e>0.1$, the eccentricity distributions of the giants and the dwarfs are essentially the same. The mean orbital eccentricities are $\langle e\rangle=$ $0.37 \pm 0.06(\sigma=0.20)$ for the red giants, and $\langle e\rangle=$ $0.40 \pm 0.03(\sigma=0.19)$ for the 40 dwarfs with $P>20$ days. Note that both the means and the standard errors are essentially the same. But when the three low-eccentricity red giant binaries are included, the K-S test indicates an $86 \%$ chance of different parent distributions. So why should some of the red giants be more vulnerable to orbital evolution?

As the primary in a binary begins to evolve, it can overflow its Roche surface and transfer mass to the secondary, if the orbit is small enough. In Carney et al. (2001), it is argued that the fairly long orbital periods and small eccentricities found for field metal-poor blue stragglers are the result of orbital evolution during stable mass transfer. Note that the crucial variable in orbital evolution is the ratio of the stellar radius to the orbital separation, so that larger stars, such as luminous red giants, may be more easily synchronized and also experience orbital circularization at longer periods than main-sequence stars (Zahn 1977, 1989; Verbunt \& Phinney 1995). The expanding radius of a star as it evolves up the RGB increases its susceptibility to tidal effects, and we therefore expect some of the red giant spectroscopic binaries to have smaller than average orbital eccentricities, with the shorter period or larger radius stars most likely to be affected. Of the stars in our program, the three red giants with $e<0.1$ are $\mathrm{BD}+30^{\circ} 2034, \mathrm{BD}+18^{\circ} 2890$, and $\mathrm{CD}$ $-37^{\circ} 14010$. Are their orbits and sizes consistent with tidal circularization having altered their orbital eccentricities? Assuming a combined system mass of $1.2 M_{\odot}$, their semimajor axes are 111,273 , and $73 R_{\odot}$, respectively, compared with the radii estimated for the primaries in Table 2 of 47, 9 , and $50 R_{\odot}$. The orbital parameters of $\mathrm{BD}+30^{\circ} 2034$ and $\mathrm{CD}$ $-37^{\circ} 14010$ are consistent with the tidal circularization model, and depending on the inclination of the rotational axis of the primary star, we should expect to see line broadening as high as about 19 and $39 \mathrm{~km} \mathrm{~s}^{-1}$. On the other hand, $\mathrm{BD}+18^{\circ} 2890$ is interesting because its estimated radius is quite small compared with the estimated separation of the components. The ratio of $a$ to $R$ is over 30, while the other two stars have ratios of less than 3 . Perhaps BD $+18^{\circ} 2890$ is an AGB star that interacted tidally with its companion when it was previously a large RGB star. An abundance analysis would help determine its evolutionary state, since some AGB stars show signs of $s$-process material in their photospheres (see Busso et al. 2001 and references therein). Or, of course, it could simply have been formed with a nearly circular orbit. HD 218732 is also interesting, because it appears to have an eccentric orbit despite having $a / R \approx 3$. At least two explanations are possible in this case. First, our estimated radius may be in error: we noted earlier that our temperature and radius estimates for this star are not as well determined as for the other stars. On the other hand, if the temperature and radius estimates are correct, then the star is very near the RGB tip and evolving rapidly. Perhaps the timescale for orbital circularization is longer than the radial expansion timescale in this case.

Will mass transfer or even a merger event happen at some point? As Table 2 indicates, and as stellar evolution models predict, low-mass, metal-poor stars evolve to reach large radii, on the order of $0.5 \mathrm{AU}\left(>100 R_{\odot}\right)$. It is worth considering the critical range of periods expected for interactions. As the more massive star ascends the RGB and swells in size, tidal interactions will increase in strength, and co-rotation may occur. This would be followed by orbital circularization and, for short enough periods, mass transfer, which may lead to the creation of blue stragglers (see Carney et al. 2001). According to Paczyński (1971), for an initial primary-to-secondary mass ratio $M_{1} / M_{2}$, Roche lobe overflow will occur if

$$
R_{1} / a \geq 0.38+0.2 \log \left(M_{1} / M_{2}\right),
$$

where $a$ is the orbital semimajor axis and $R_{1}$ is the radius of the primary. For the system,

$$
\log P=1.5 \log a-0.5 \log \left(M_{1}+M_{2}\right)-0.936,
$$

where $P$ is in days, $a$ is in solar radii, and the masses are in solar units. Equation (3) indicates that $\mathrm{BD}+30^{\circ} 2034$ and $\mathrm{CD}-37^{\circ} 14010$ are on the threshold of mass transfer and are probably undergoing relatively rapid orbital evolution.

\subsection{Secondary Masses}

Lacking eclipses or astrometric information about the orbital inclination, single-lined spectroscopic binaries do not reveal the masses of their companion stars except by a statistical analysis of a well-defined population of binaries, and even then only when reliable estimates are available for the primary masses. For our sample of metal-poor field red giants, we may assume that the mass of every primary star is roughly $0.8 M_{\odot}$. We may therefore use equation (2) to calculate a lower limit to the mass of the secondary, for $\sin i=1$. These minimum masses are summarized in Table 5 and plotted in Figure 7. The rightmost dotted line corresponds to the value of $0.8 M_{\odot}$ adopted for the primary mass. As 


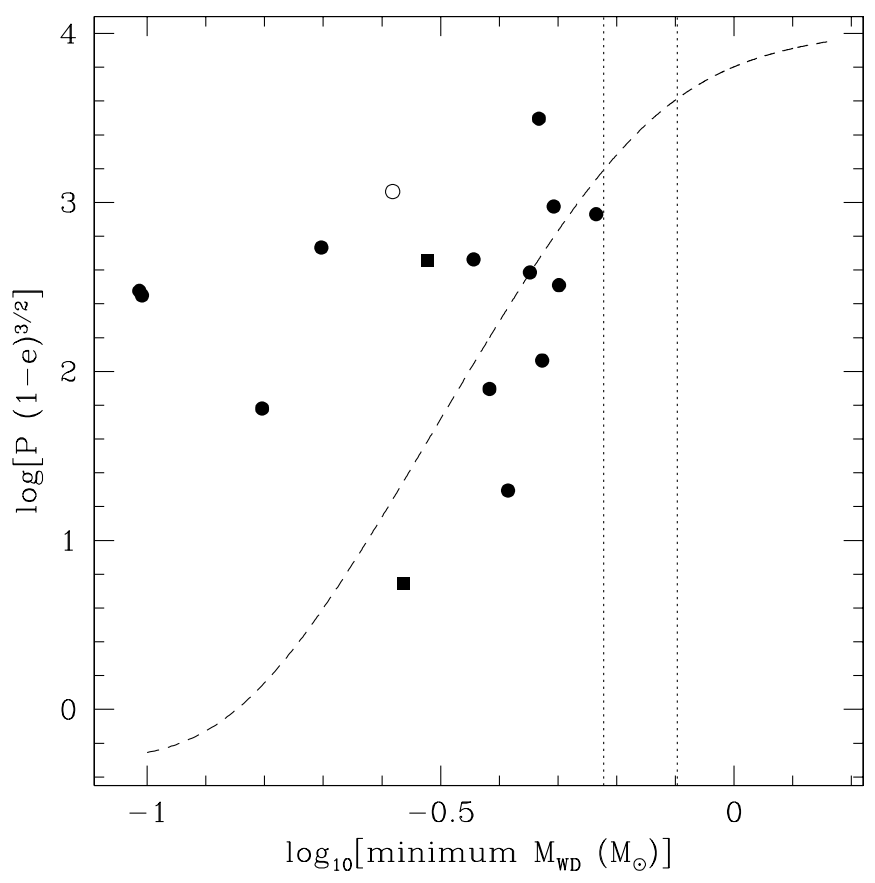

FIG. 7.-Theoretical minimum masses for white dwarfs resulting from stable mass transfer (dashed line; from Rappaport et al. 1995). The right dotted line marks the turnoff mass for globular cluster stars. The left dotted line marks the approximate mass expected for normal halo white dwarfs where mass transfer has not been involved. The points are minimum masses derived from the orbital solutions. Filled circles are new results from Table 5 , and squares are literature solutions from the same table. The open circle is the giant $\mathrm{CH}$ star HD 135148.

expected, all minimum secondary masses lie to the left of this line. The minimum secondary masses are also all smaller than the nominal mass of a white dwarf that results from the normal evolution of an isolated low-mass star, $M_{\mathrm{WD}} \approx 0.6 M_{\odot}$. The results are thus consistent with the secondaries' being normal white dwarfs or main-sequence stars.

But what about evolution in a mass transfer system? This may be expected for close binaries in which the original primary is more massive than $0.8 M_{\odot}$. As the primary evolves, it may fill its Roche surface and start transferring mass onto the secondary. The first stage of mass transfer, while the donor star is still more massive than the receiving star, proceeds very rapidly because the orbit shrinks as the mass ratio changes. After the donor star becomes less massive than its companion, the orbital evolution reverses and the orbital separation grows rather than shrinking. This stage of mass transfer proceeds much more slowly, because the system has to wait for evolution of the donor star to keep its Roche surface filled as the orbit expands. Eventually the donor star is completely stripped of its outer layers, leaving only a degenerate core. At this point, the orbital evolution of the system stops, with the orbital separation and therefore the period set by the mass of the degenerate core. In this model of stable and conservative mass transfer, we expect a tight correlation between the mass of the remnant white dwarf and the orbital period.

Rappaport et al. (1995) have modeled the evolution of binaries undergoing stable mass transfer, but for the case in which the recipient is a neutron star. The same physics should also apply to systems where the recipient is a white dwarf. In Figure 7, we show the model predictions of Rappaport et al. as a dashed line. We draw the white dwarf mass as a function of the orbital period and eccentricity, $\log \left[P(1-e)^{3 / 2}\right]$. The essence of the model is that shortperiod systems are so close that mass transfer begins early, when the red giant core has not yet grown to a "mature" size, and so the resultant white dwarf will appear with a small, "premature" mass. We point out that we have chosen the coefficient $R_{0}$ in the model to be $3300 R_{\odot}$, consistent with the Population II case discussed by Rappaport et al. If all the secondary stars were white dwarfs and had evolved via stable mass transfer, their minimum masses, plotted in Figure 7, should all lie to the left of the dashed line. The fact that many of the stars have moderately short periods but minimum masses larger than permitted by this theory suggests that many of the secondary stars in our sample of spectroscopic binaries are main-sequence stars. This is, of course, expected to be the case.

For CD $-37^{\circ} 14010$, we may make a reasonable estimate of its orbital inclination and hence its secondary mass. CD $-37^{\circ} 14010$ is a luminous red giant or AGB star with a relatively short period binary companion $(P=65$ days). Presumably, the very low orbital eccentricity for this system was caused by tidal interactions, which force, first, alignment of the orbital and rotational axes, then synchronization of the axial rotation with the orbital motion, and, finally, orbital circularization. Because the orbit has been circularized, we assume that the rotational period of the star is the same as the orbital period. For an estimated primary radius of $50 R_{\odot}$, the implied rotational velocity is $39 \mathrm{~km} \mathrm{~s}^{-1}$, which is consistent with the measured line broadening of $v_{\text {rot }} \sin i=19 \mathrm{~km} \mathrm{~s}^{-1}$ (see $\S 8$ and Table 4) if the rotational axis is inclined to our line of sight by $29^{\circ}$. If the orbital plane is the same as the star's equatorial plane, the assumption of a primary mass of $0.8 M_{\odot}$ and the measured mass function lead to an estimate for the secondary's mass of somewhat less than $0.4 M_{\odot}$. This could be a case of a "premature" white dwarf of unusually low mass as predicted by the stable mass transfer scenario, or the secondary could be a normal $\mathrm{M}$ dwarf. This is an important point in the study of the possible relationship between stellar rotation and mixing in evolved metal-poor red giants, because it means that any abundance anomalies present in the atmosphere of this star are unlikely to have been contributed by its companion, if it proves to be a main-sequence star. If anomalies are found, then the relatively high rotational velocity of this star (close to $40 \mathrm{~km} \mathrm{~s}^{-1}$ when the correction is made for sin $i$ ) may suggest a cause-and-effect relationship between high rotation and deep internal mixing. Detailed abundance analyses of this star are recommended (and are underway).

\subsection{CH Stars}

Most, if not all, $\mathrm{CH}$ stars have proved to be members of binary systems in which the secondaries are probably white dwarfs (see McClure \& Woodsworth 1990; McClure 1997). Both of the CH stars in our program, HD 135148 and BD $-1^{\circ} 2582$, are spectroscopic binaries. An adopted primary mass of $0.8 M_{\odot}$ and the derived mass function for HD 135148 indicate that the minimum mass for the companion star is $0.26 M_{\odot}$, comfortably below $0.6 M_{\odot}$, the approximate mass of a white dwarf that has evolved without experiencing mass transfer. The value is also well below the stable mass transfer model predictions of Rappaport et al. (1995), as 
shown in Figure 7, where HD 135148 is plotted as an open circle.

\section{VELOCITY JITTER}

In their detailed study of radial velocities of red giants in the metal-poor globular cluster M3 $([\mathrm{Fe} / \mathrm{H}]=-1.46$; Kraft et al. 1993, 1995), Gunn \& Griffin (1979) noted that many of the most luminous stars showed higher than expected dispersions in their radial velocity measurements. A more extensive study of the cluster by Pryor, Latham, \& Hazen (1988) found similar results, with the greatest velocity variations seen within $0.5 \mathrm{mag}$ (in $V$ ) of the RGB tip $\left(M_{V}<-1.8\right)$. The radial velocity variability does not appear to be associated with orbital motion and has been called velocity jitter.

There have been numerous studies of red giants in other globular clusters searching for clues for the source of the velocity jitter. Among clusters of comparable metallicity, M13 $([\mathrm{Fe} / \mathrm{H}]=-1.48 ;$ Kraft et al. 1997) and M22 $([\mathrm{Fe} / \mathrm{H}]$ $=-1.65$; Brown \& Wallerstein 1992), more evidence of jitter has been found. Lupton, Gunn, \& Griffin (1987) noted such variations especially for the stars in M13 with $M_{V} \leq-1.5$. Some of these stars are also known to be photometric variables. More recently, Côté et al. (1996) showed the presence of such velocity variability in M22, at a roughly constant amplitude for stars within $0.5 \mathrm{mag}$ of the RGB tip $\left(M_{V}<-1.8\right)$ and declining to undetectable levels near the magnitude of the horizontal branch $\left(M_{V} \approx+0.5\right)$.

While luminosity appears to be an important parameter for the appearance of jitter, metallicity does not. Jitter has been seen in the more metal-rich cluster 47 Tuc by Mayor \& Mermilliod (1984), a cluster with $[\mathrm{Fe} / \mathrm{H}]=$ -0.73 (Gratton, Quarta, \& Ortolani 1986; Brown \& Wallerstein 1992; Norris \& Da Costa 1995). Again, the jitter seems to be concentrated in the more luminous stars, those with $M_{V}<-1.2$. Finally, the "multimetallicity" cluster $\omega$ Cen $(\langle[\mathrm{Fe} / \mathrm{H}]\rangle \approx-1.6$, but ranging from -0.8 to -1.8 ; Norris \& Da Costa 1995) also shows such jitter among its red giants. Mayor et al. (1997) found that a minority of the stars in the cluster with $V<12.3 \mathrm{mag}$ $\left(M_{V}<-1.4\right)$ are low-level radial velocity variables, with $\sigma\left(v_{\text {rad }}\right) \approx 1.5-3 \mathrm{~km} \mathrm{~s}^{-1}$. Unfortunately, none of the above studies of cluster giants had enough observations per star to search for periodicities in the velocity variability that might be coupled to rotational or pulsational properties of the stars.

Which stars in our program show signs of velocity jitter? Consideration of Figure 2 provides our primary means of identifying such stars. Figure $2 c$, in particular, includes only the lowest values of $P\left(\chi^{2}\right)$, such that at most only a few constant-velocity stars should appear. [Note that none of the $P\left(\chi^{2}\right)$ values plotted in Fig. 2 include compensation for the orbital solutions. Using only mean velocities, all the binary stars have $P\left(\chi^{2}\right)<10^{-6}$.]

To more carefully explore the occurrence of velocity jitter, we return now to the $P\left(\chi^{2}\right)$ values computed with compensation for orbital motion, and we plot in Figure 8 the logarithm of $P\left(\chi^{2}\right)$ versus absolute visual magnitude $M_{V}$. Stars not recognized as binaries but with $P\left(\chi^{2}\right) \leq 0.001$ are plotted as filled circles. All the other nonbinary stars with $P\left(\chi^{2}\right)>0.001$ are plotted as open circles. Similarly, the binary stars are plotted as triangles, open for the larger values of $P\left(\chi^{2}\right)$, and filled for values smaller than 0.001 . Almost

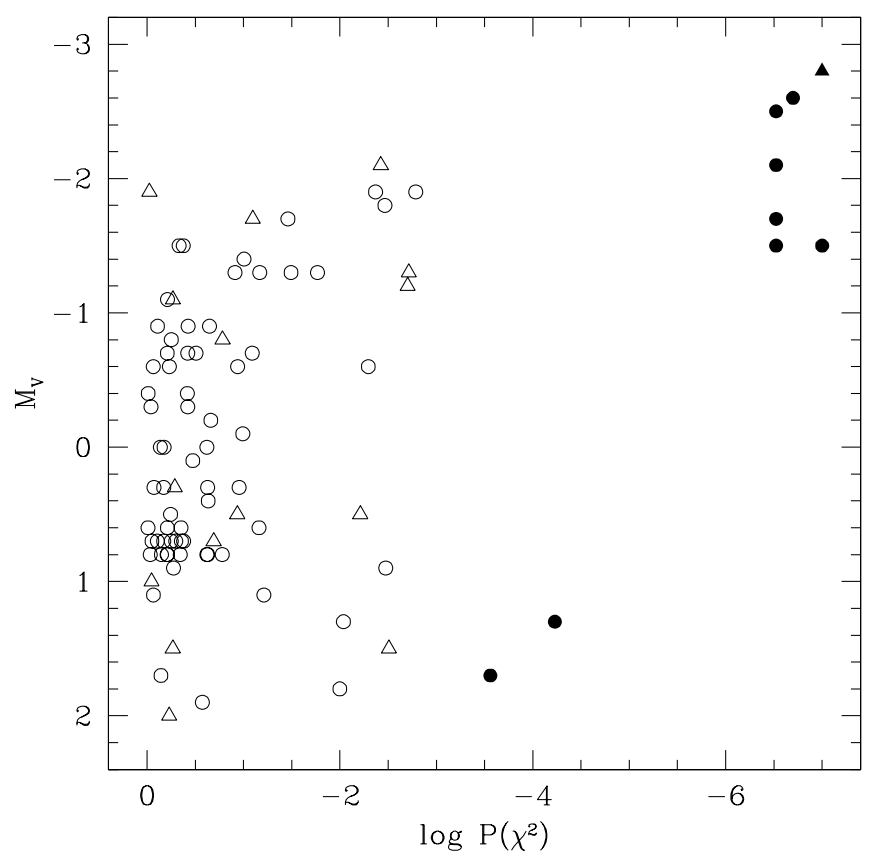

FIG. 8.- Logarithms of the $P\left(\chi^{2}\right)$ values plotted against the estimated absolute magnitudes. Nonbinary stars with $P\left(\chi^{2}\right) \leq 0.001$ are plotted as filled circles and are assumed to be displaying velocity jitter. All other nonbinary stars are plotted as open circles. The spectroscopic binary stars' $P\left(\chi^{2}\right)$ values have been computed using the residuals from the orbital solutions. The binary stars are represented by triangles.

all the stars with very small $P\left(\chi^{2}\right)$ values probably manifest velocity variability, and most of them have high luminosities. First, however, let us consider the two stars with intermediate $P\left(\chi^{2}\right)$ values and relatively low luminosities, HD 97 and HD 213467. We show their velocity histories in Figure 9. For HD 97, the low value for $P\left(\chi^{2}\right)$ is caused by two outlying velocities among the 30 otherwise roughly constant measures. Without those two velocities, $P\left(\chi^{2}\right)$ becomes 0.41 . Either the star also shows velocity jitter but only very rarely, one or both of the velocity measures have unusually large errors, or the star is a low-amplitude spectroscopic binary with a highly eccentric orbit. For HD 213467, more observations are needed in order to see whether the velocity variability persists and, if so, to see whether it is periodic. If orbital motion is confirmed for either of these two stars, the small amplitudes imply lowmass secondaries or very unfavorable (and unlikely) viewing angles.

The six nonbinary stars that appear to display velocity jitter are HD $3008, \mathrm{BD}-18^{\circ} 271, \mathrm{BD}+22^{\circ} 2411$, and $\mathrm{HD}$ 110281, 121261, and 214925. Among the binary stars, HD 218732 has an extremely low value of $P\left(\chi^{2}\right)$, and this star probably is manifesting velocity jitter as well. As in the case of the globular cluster stars that have been found to show velocity jitter, all our candidates are luminous, low-gravity stars, with $M_{V} \leq-1.2, T_{\text {eff }} \leq 4550 \mathrm{~K}, R>30 R_{\odot}$, and $\log g \leq 1.3$. In total, seven of the 17 stars $(41 \% \pm 16 \%)$ with $M_{V} \leq-1.2$ show clear signs of velocity jitter.

Can we identify the underlying physical cause of the jitter? Three of the above six stars are already known to be photometric variables. Hipparcos observations of HD 3008 and HD 110281 proved them to be variable (and they have been designated CM Cet and KR Vir, respectively). The 


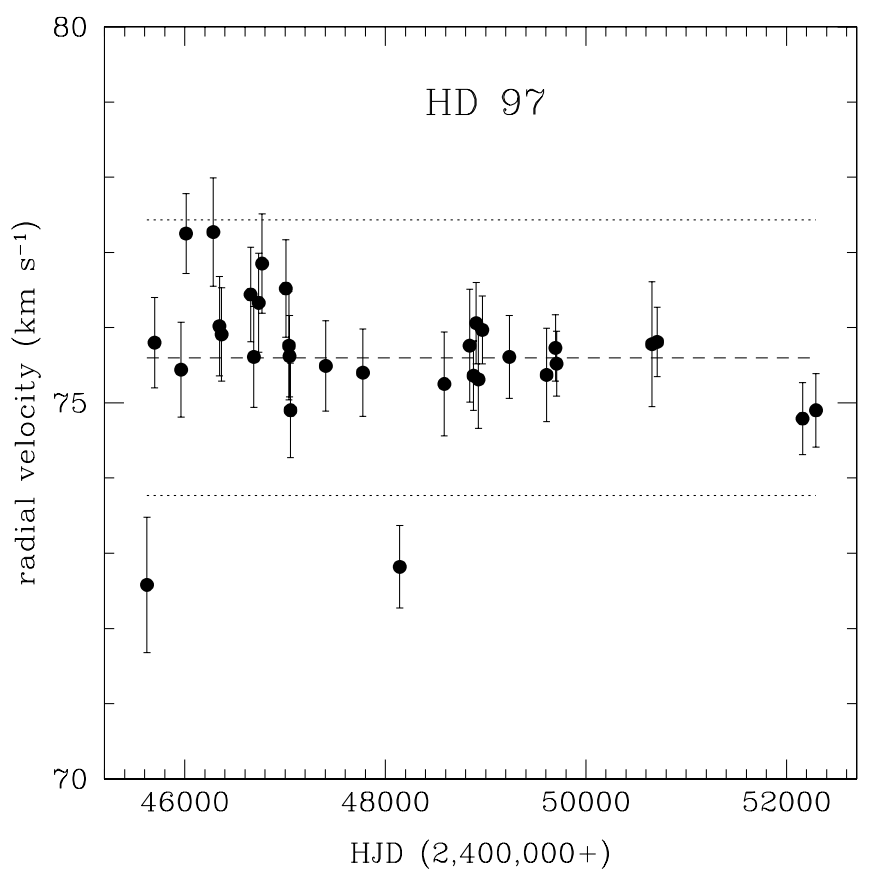

FIG. $9 a$

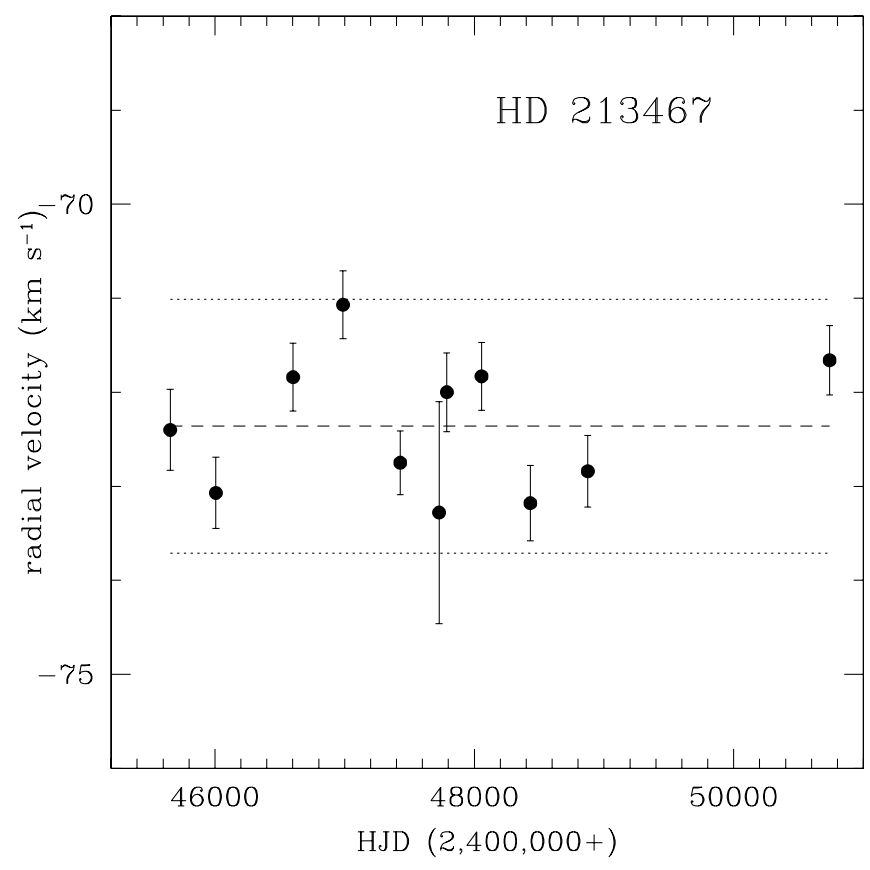

FIG. $9 b$

FIG. 9.-Velocity histories of two stars that have very low $P\left(\chi^{2}\right)$ values yet are of relatively low luminosity: (a) HD 97 and $(b)$ HD 213467 . The mean velocities, as well as $\pm 3 \sigma_{\text {int }}$, are shown as horizontal lines.

amplitudes and standard errors for these two stars are $A=0.134 \mathrm{mag}$ and $A=0.195 \mathrm{mag}$, respectively, and $\sigma=0.032 \mathrm{mag}$ and $\sigma=0.045 \mathrm{mag}$, respectively. Rufener \& Bartholdi (1982) found HD 214925 to be variable, and this was confirmed by Hipparcos $(A=0.156 \mathrm{mag}, \sigma=0.033$ $\mathrm{mag}$ ). The variability does not appear to be due to eclipses, but starspots or radial pulsation could explain such photometric variations.

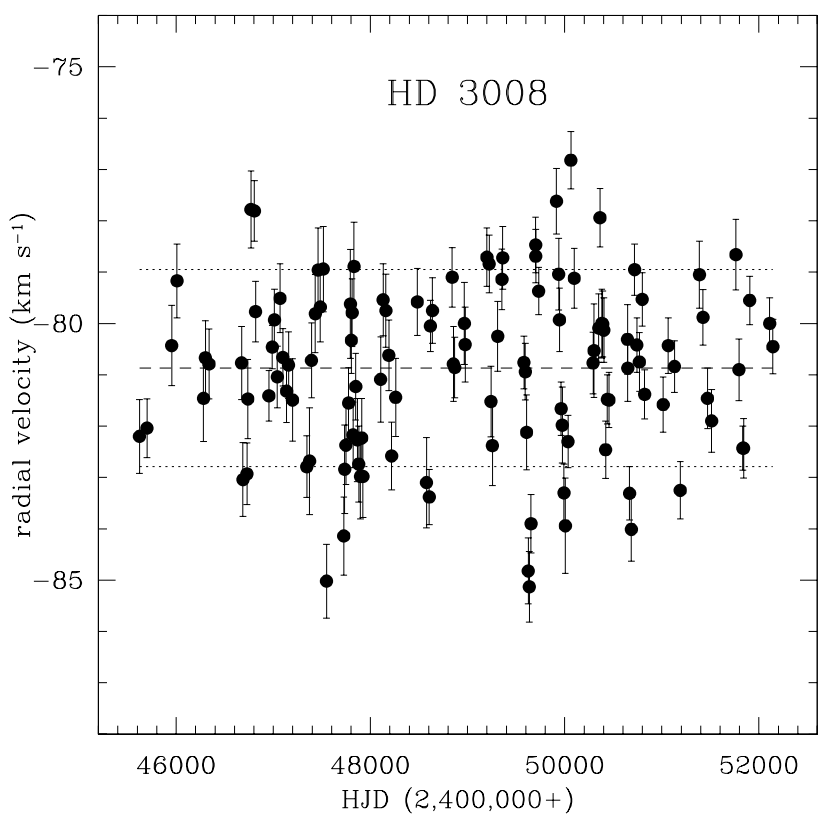

FIG. $10 a$
A second piece of evidence comes from our two beststudied cases, HD 3008 (123 velocities covering $17.9 \mathrm{yr}$ ) and $\mathrm{BD}+22^{\circ} 2411$ (153 velocities covering $15.1 \mathrm{yr}$ ). Both stars are estimated to have high luminosity $\left(M_{V} \approx-1.5\right.$ and $M_{V} \approx-1.7$, respectively). We show the velocity histories of both stars in Figure 10. The mean velocities are the dashed lines, and the $\pm 3 \sigma_{\text {int }}$ limits are the dotted lines. As Table 4 shows, the $P\left(\chi^{2}\right)$ values for both stars are extremely low, of

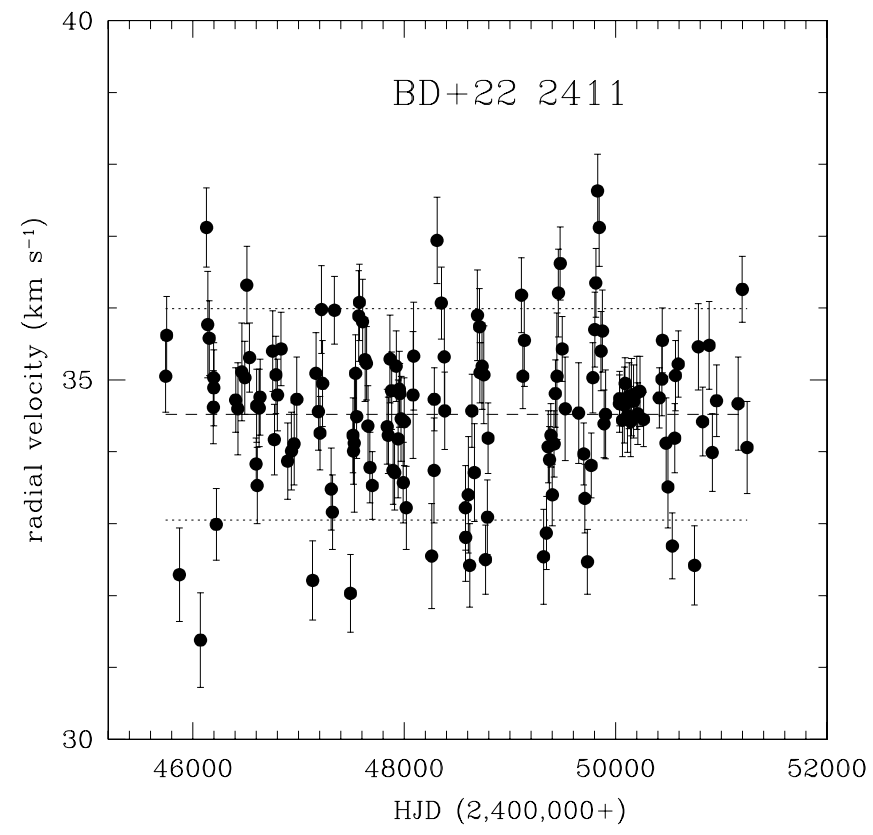

FIG. $10 b$

FIG. 10. - Velocity histories of the two stars showing velocity jitter that have the most measurements: $(a)$ HD 3008 and $(b)$ BD $+22^{\circ} 2411$. The mean velocities, as well as $\pm 3 \sigma_{\text {int }}$, are shown as horizontal lines. 
the same magnitude as those stars we have found to have spectroscopic binary companions. Formally, we were able to obtain " orbital solutions" for both stars, with periods of $172.1 \pm 0.3$ and $185.9 \pm 0.5$ days, respectively. In both cases the velocity amplitude of the orbital solution is small, 1.55 and $0.96 \mathrm{~km} \mathrm{~s}^{-1}$, respectively, and the minimum masses implied for the secondaries are substellar, 0.034 and 0.017 $M_{\odot}$, respectively, using the entire velocity sets.

However, we do not believe that the velocity variations exhibited by HD 3008 and $\mathrm{BD}+22^{\circ} 2411$ are due to orbital motion, because the orbital solutions are not robust, despite a large number of high-quality velocity data. Despite such data, the derived orbital parameters have relatively large error estimates. Perhaps the primary evidence that the solutions are not credible is that the observed velocity variations appear to come and go over timescales of years. For example, when we divide the data for HD 3008 into a few subsets, each with several dozen velocities and sufficient coverage to determine an orbital solution, one subset shows a very strong peak in the power spectrum and a convincing orbital solution with a period of 174 days, while another subset shows several weak candidate periods, and a third subset shows no peak at all in the power spectrum. This behavior is much more reminiscent of a rotating star with spots that come and go than of either orbital motion or radial pulsation.

Another reason why pulsation appears to be an unlikely cause of the velocity variations observed in HD 3008 and $\mathrm{BD}+22^{\circ} 2411$ is provided by the pseudoperiods that we find, 172 and 186 days. These are longer than any pulsational periods found for red giants in metal-poor globular clusters. The 31 such variables known in NGC 2419, 5024, $5272,6205,6121,6715$, and 7006 have periods ranging from about 40 days up to about 150 days. The longer periods found for the variations in HD 3008 and $\mathrm{BD}+22^{\circ} 2411$ again do not appear to be consistent with pulsation.

To explore further the possibility that the velocity variations observed for HD 3008 might be due to radial pulsation or rotation coupled with spots (both mechanisms should lead to brightness variations), we looked for correlations between our velocities and the 80 photometric measurements reported from Hipparcos, which have a range of 0.13 mag and a typical internal error of $0.02 \mathrm{mag}$. When subjected to a power spectrum analysis, the Hipparcos observations show two candidate periodicities: a fairly prominent one at 176.8 days, very close to the "orbital" period found for the velocities, and an even stronger peak at half that period, 88.6 days. We employed 66 velocities spanning 2754 days centered on the 980 day span of the Hipparcos observations. (The relevant velocities cover HJD 2,447,344 to 2,450,098.) When we phase the Hipparcos photometry to match an "orbital" solution for the photometry over the same epoch, as shown in Figure 11, we find that there is an uncomfortably large gap in the phase coverage in the photometry near what we assume to be maximum light. Nevertheless, there is a suggestive dip in the brightness near phase $\phi=0.15$. Are pulsation models consistent with these data?

In the case of pulsation, systemic radial velocity occurs when the star is at either maximum or minimum radius. Considering Figure 11 (top), these occur for HD 3008 at $\phi \approx 0.15$ and $\phi \approx 0.75$, respectively, if the star is pulsating. Unfortunately, the photometry for HD 3008 does not clearly reveal a phase of maximum light, so we focus on the
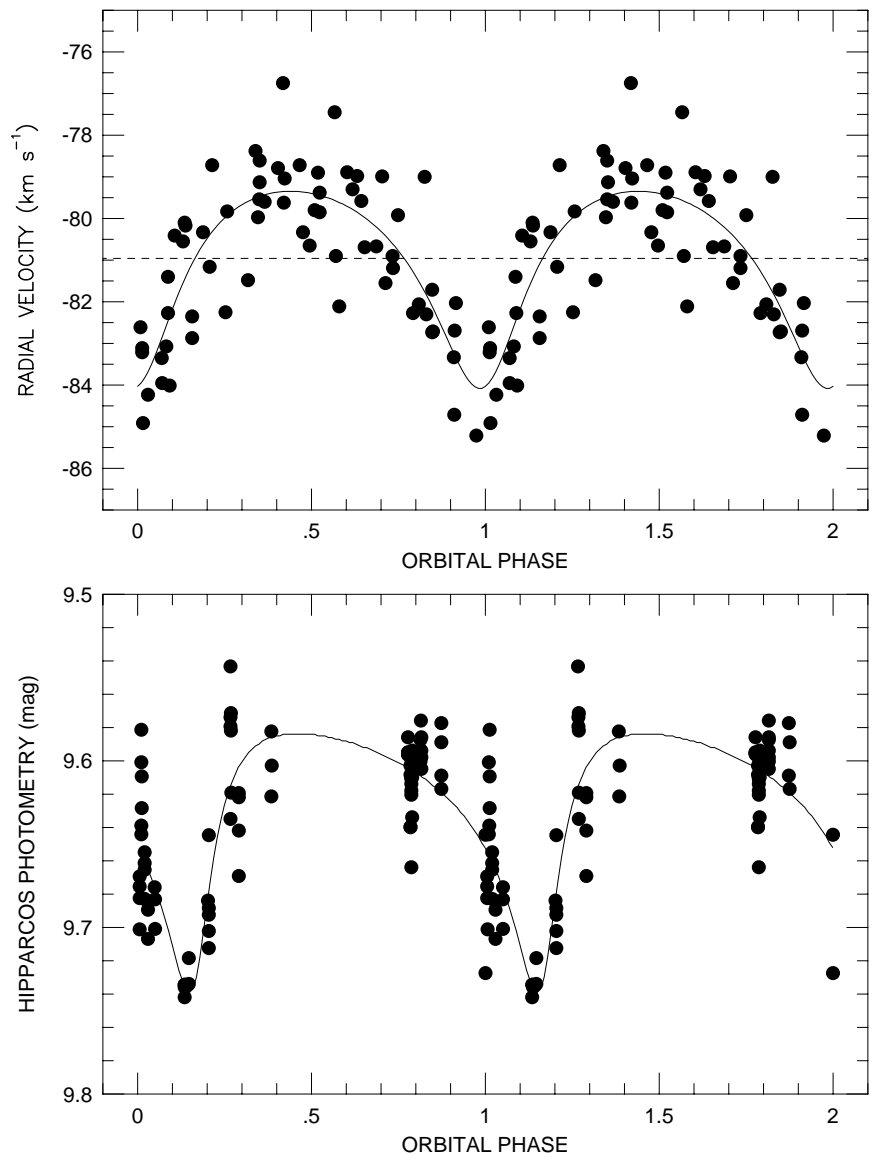

FIG. 11.-Comparison of the phased velocity and photometric data for HD 3008, as described in the text. In both cases, formal solutions to the variations are shown. If the velocity variations are due to pulsation, maximum radius would occur at $\phi \approx 0.15$ and minimum radius at $\phi \approx 0.75$.

timing of minimum light relative to minimum and maximum radius. Figure 11 shows that for HD 3008, minimum light occurs at about the same phase as maximum radius, $\phi \approx 0.15$, which is not consistent with normal pulsating variable stars. Specifically, for RR Lyrae, Cepheid, and Mira variables, maximum optical light occurs when the temperature is hottest, which occurs very shortly after minimum radius. In the case of RR Lyrae variables, the analyses of Jones, Carney, \& Latham (1988), Fernley et al. (1989), and Liu \& Janes (1990) show that for fundamental-mode pulsators (RRab variables), minimum light occurs about 0.5 cycles away from maximum radius, completely out of phase with the behavior seen in Figure 11. For lower density Cepheid variables, minimum light follows maximum radius by 0.4 cycles, using the roughly contemporaneous radial velocities and photometry for stars with periods exceeding 7 days (to avoid overtone pulsators) reported by Moffett \& Barnes $(1985,1987)$ and Barnes, Moffett, \& Slovak (1987). Unpublished results for the 25.7 day type II Cepheid V42 in the globular cluster M5 (B. W. C. \& D. W. L.) show maximum light preceding minimum radius by about 0.03 cycles and minimum light following maximum radius by 0.2 cycles. The even lower density Mira variables are more difficult to analyze, since their atmospheres are greatly extended and the presence of shock waves often leads to line doubling. Line profiles are also complicated by stellar winds. Nonetheless, the early work of Joy (1954) and others, 
summarized by Hoffmeister, Richter, \& Wenzel (1985), and infrared spectroscopy by Hinkle, Scharlach, \& Hall (1984), indicates that minimum optical light follows maximum radius by about 0.3 cycles. None of these pulsators therefore conform to the behavior seen in Figure 11. One interesting point is that fundamental-mode pulsations tend to produce asymmetric light and velocity curves, at least in RR Lyrae and Cepheid variables, while Figure 11 shows a more sinusoidal behavior. Overtone pulsators yield more symmetric light and velocity curves, but this would make the fundamental pulsational period even longer than 172 days, and even more at odds with what is observed in the globular cluster variables. We conclude that pulsation models fail to explain the velocity and photometry data for HD 3008 .

We are left with starspots, coupled with rotational modulation, as a possible explanation for the velocity jitter, but here the tests are less useful because they are dependent on the adopted spot geometries. Let us consider the simplest case, with one very large spot with a lifetime on the order of a stellar rotation period or longer. Velocity shifts can result when a bright or dark spot dominates either the approaching or the receding limb of a star. Changes in the size, number, and location of the starspots might be able to explain why the velocity modulations are more pronounced at some epochs. The idea of a single large spot or a sample of a few such large spots is plausible because luminous, cool, lowgravity stars may have exceptionally large convective cells (Schwarzschild 1975). In the case of a single spot, minimum brightness should occur when the dark spot is centered on the side of the star facing us. This is also a phase when the spot has little effect on the velocity deduced for the star, because the spot is moving across the line of sight. Thus, minimum light should occur when the measured radial velocity is near the mean. Although the minimum light occurs near the mean velocity, as seen in Figure 11, it is at the wrong mean velocity crossing. Minimum light should occur when the velocity curve is crossing the mean toward more blueshifted values, because a dark spot removes more light from the continuum than from the absorption lines, as described by Vogt \& Penrod (1983). This therefore weakens the contributions of the hemisphere containing the spot to the overall flux spectrum of the star, so that a spot on the approaching hemisphere actually induces a redward shift in the absorption-line spectrum. This does not agree with the observations, and we conclude that the minimum light for HD 3008 occurs at the phase opposite that predicted by a simple spot model. A similar analysis for a single bright spot reaches the same conclusion. Are other models capable of explaining the observations? The photometric gap in the coverage presents us with problems, of course, but there may be models involving brighter, hotter, upwellings surrounded by darker "downdrafts" that may yet provide a satisfactory match to the observations. We recommend further observations of HD 3008 and $\mathrm{BD}+22^{\circ} 2411$ to further explore starspots as the cause of velocity jitter.

We conclude that neither orbital motion nor radial pulsation can explain the velocity jitter that we observe among the most luminous red giants. An additional argument against these two mechanisms is that we do not find a strictly periodic variation for any of the stars showing velocity jitter. Even for the most promising example, HD 3008, the velocity variations appear to come and go and are not strictly periodic. If we must choose among orbital motion, pulsation, and rotational modulation to explain the perio- dicities seen for the velocities of HD 3008 and BD $+22^{\circ} 2411$, we are forced to adopt rotational modulation, even though the simplest model involving large, dark spots fails to reproduce the observations.

If the velocity variations observed in HD 3008 and BD $+22^{\circ} 2411$ are ascribed to rotational modulation, the equatorial rotational velocities implied by our estimated radii and spectroscopic periods are surprisingly large for physically extended, cool, luminous, highly evolved red giants: $14.5 \mathrm{~km} \mathrm{~s}^{-1}$ for HD 3008 and $12.7 \mathrm{~km} \mathrm{~s}^{-1}$ for BD +22 2411 . Our comparisons of the observed and synthetic spectra discussed in the following section resulted in values of 9.2 and $7.3 \mathrm{~km} \mathrm{~s}^{-1}$ for $v_{\text {rot }} \sin i$, which are marginally within our ability to measure but are consistent with the large rotational velocities implied for these stars if the rotational axes are inclined with respect to the plane of the sky by $39^{\circ}$ and $35^{\circ}$, respectively.

\section{LINE BROADENING}

We have estimated the stellar rotational velocities, $v_{\text {rot }} \sin i$, using our synthetic spectra for all of our program stars. As described in $\S 4.4$, for each star, we selected the synthetic spectrum for use as a template that best matches the derived temperature, gravity, and metallicity parameters and then chose the rotational broadening value that produced the highest average correlation value. (For BD $+44^{\circ} 493$, we employed templates that produced the best correlations, which differed somewhat from those derived from the photometry, as explained earlier.) To estimate the stellar rotational velocity, we interpolated including the correlation values for the two velocities adjacent to the preferred value using a quadratic fit. The derived $v_{\text {rot }} \sin i$ values are listed in the final column of Table 4 . The typical standard error in these values derived from the individual spectra ranged from 0.5 to $2.0 \mathrm{~km} \mathrm{~s}^{-1}$, so the mean values are well determined, at least internally.

We undertook a series of numerical experiments to assess how sensitive the derived line-broadening values are to the parameters of the adopted synthetic spectrum templates. (Recall the case of BD $+44^{\circ} 493$ discussed in $\S 4.4$, where a change of $500 \mathrm{~K}$ in $T_{\text {eff }}, 1.0$ dex in $\log g$, and 0.5 dex in $[\mathrm{Fe} / \mathrm{H}]$ changed the derived value of $v_{\text {rot }} \sin i$ from 3.9 to 3.1 $\mathrm{km} \mathrm{s}^{-1}$.) The results are most accurately described by differences taken in quadrature between the derived velocities. For example, the adopted template for HD 20 in Table 2 resulted in $v_{\text {rot }} \sin i=5.9 \mathrm{~km} \mathrm{~s}^{-1}$. Use of a template $250 \mathrm{~K}$ hotter ("at5500g $25 \mathrm{~m} 15$ ") resulted in $v_{\text {rot }} \sin i=6.6 \mathrm{~km}$ $\mathrm{s}^{-1}$, for a nominal sensitivity of $3.0 \mathrm{~km} \mathrm{~s}^{-1}$ for an error in temperature of $250 \mathrm{~K}$. The full run of experiments showed that changes of $250 \mathrm{~K}$ in temperature, 0.5 dex in gravity, or 0.5 dex in metallicity individually led to quadrature velocity sensitivities of $3.0,3.2$, and $3.3 \mathrm{~km} \mathrm{~s}^{-1}$, independent of the derived rotational velocity, and roughly independent of the direction of the change. Uncertainties in the stellar atmospheric parameters and errors associated with interpolation in the grid of synthetic spectra probably account for the nonzero but small $\left(\approx 5 \mathrm{~km} \mathrm{~s}^{-1}\right)$ rotational velocities derived for many of our program stars. While we have referred to these values as " rotational velocities," the reader should be aware that a number of other systematic effects in the mismatch between the synthetic and observed spectra could lead to larger values of $v_{\text {rot }} \sin i$ than expected. For example, the synthetic spectra were computed using a turbulent 
velocity of $2 \mathrm{~km} \mathrm{~s}^{-1}$, but low-gravity stars may have higher turbulent velocities, and the low value employed in the synthetic spectra would lead to derived line-broadening values systematically too high in the comparison with the observed spectra. Values that are well below the instrumental resolution are more vulnerable to these uncertain systematic effects. Nonetheless, some of our stars are clearly broad-lined.

\section{DISCUSSION}

\subsection{Trends with Luminosity}

Figure 12 shows the derived line broadening (called rotational velocity) as a function of our estimated absolute visual magnitude, and we have drawn as a dashed line our instrumental resolution $\left(8.5 \mathrm{~km} \mathrm{~s}^{-1}\right)$ to help the reader distinguish between results more and less vulnerable to systematic effects. We have plotted HD 3008 and BD $+22^{\circ} 2411$ as squares using the rotational velocities derived from the pseudoperiods in their radial velocities and their estimated radii, as described at the end of $\S 7$. Binary stars are plotted as open circles. To help distinguish the hydrogen shell burning RGB stars from the core helium burning RHB stars that represent the subsequent evolutionary stage, we have plotted all the latter stars as open triangles in Figure 12, except for the binary star HD 108317, which we show as an inverted open triangle. Three features stand out in Figure 12.

The first feature of note is the general absence of a trend with luminosity. This suggests that the differences in turbulence or other forms of systematic error in the derived values

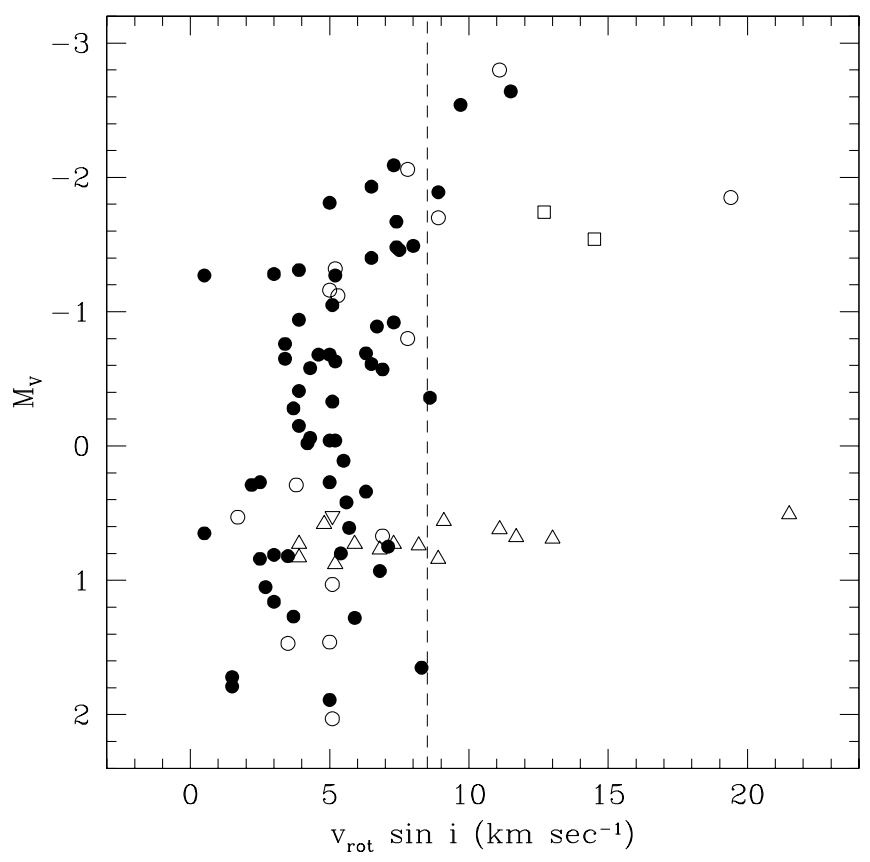

FIG. 12.-Estimated line broadening in the spectra of the program stars, plotted as a function of luminosity. We have plotted HD 3008 and BD $+22^{\circ} 2411$ as squares and with $v_{\text {rot }}$ values implied by the periodicity in their velocity jitter. Open circles identify the stellar binaries in the sample. Triangles are used to show the RHB stars enclosed within the parallelogram of Fig. 1. (The inverted triangle is the RHB binary star, HD 108317.) The dashed line indicates our instrumental resolution of $8.5 \mathrm{~km} \mathrm{~s}^{-1}$. Errors from template mismatches are a few kilometers per second, but these are added in quadrature to the derived $v_{\text {rot }} \sin i$ values. of $v_{\text {rot }} \sin i$ are probably not functions of luminosity (and, hence, functions of temperature or gravity). We note however that while a significant number of our stars show rotational velocities of less than $5 \mathrm{~km} \mathrm{~s}^{-1}$, such values are not found at the higher luminosities, $M_{V}<-1.4$. This may indicate that some form of increased, and unaccounted for, turbulence has appeared among the most luminous stars. Increasing the turbulence to $4 \mathrm{~km} \mathrm{~s}^{-1}$ for stars more luminous than $M_{V}=-1.5$ fills in the "gap" of luminous stars with zero or near-zero rotational velocities, but the effect is too small to significantly alter the derived rotational velocities for stars with $v_{\text {rot }} \sin i>8 \mathrm{~km} \mathrm{~s}^{-1}$. For example, increasing the turbulence by $4 \mathrm{~km} \mathrm{~s}^{-1}$ reduces the inferred $v_{\text {rot }} \sin i$ value for HD 214925 from 9.7 to $8.8 \mathrm{~km} \mathrm{~s}^{-1}$.

A second and very important feature is that a large fraction of the luminous red giants show significant rotation. Consider first the fainter stars, with $M_{V}>1.0$, whose radii range from about 5 to $9 R_{\odot}$. The luminous red giants with $M_{V} \leq-1.5$ are roughly 10 times larger, with radii from 50 to over $100 R_{\odot}$. Even granting a typical rotational velocity as high as $5 \mathrm{~km} \mathrm{~s}^{-1}$ to the fainter group of stars, in the simple case of constant surface angular momentum, the luminous red giants should have no detectable surface rotation, given our instrumental resolution. But three (HD 110281, BD $+8^{\circ} 2856$, and HD 214925) of the 15 nonbinary stars in our sample of red giants with $M_{V} \leq-1.4$ show $v_{\text {rot }} \sin i$ values at levels equal to or above that of our instrumental resolution. The percentage of nonbinary rapid rotators rises further when the implied rotational velocities HD 3008 and BD $+22^{\circ} 2411$ are included.

A third point is to answer the question whether the rotation is related to jitter, as apparently is the case for HD 3008 and $\mathrm{BD}+22^{\circ} 2411$. Excluding the three luminous spectroscopic binary stars, $\mathrm{BD}+30^{\circ} 2034, \mathrm{CD}-37^{\circ} 14010$, and HD 218732 , the three remaining rapidly rotating stars, HD 110281, BD $+8^{\circ} 2856$, and HD 214925, are all described above as showing signs of velocity jitter because of their very small values of $P\left(\chi^{2}\right)$.

\subsection{Possible Causes of Rotation}

We begin with a discussion of the probability of the rotational velocities' arising from an earlier stage of evolution. We then look at induced rotation, specifically, if there are signs of binary-related interactions in our data and, second, if binary companions are common enough among metalpoor stars to provide a supply of angular momentum donors. We explore transport of internal angular momentum at the end of this section.

\subsubsection{Inherited Rotation}

Rapidly spinning main-sequence stars must evolve to become more slowly rotating red giants if surface angular momentum is conserved, so our first question is, what fraction of metal-poor dwarfs rotate rapidly? Actually, we must rephrase the question because the rapidly rotating stars found by Latham et al. (2002) resist our methods of metallicity estimation. But the fraction is likely to be very small. Of the 1354 stars with reported rotational velocities, only $2 \%$ had derived $v_{\text {rot }} \sin i$ values of $8.5 \mathrm{~km} \mathrm{~s}^{-1}$ or greater. Perhaps the strongest evidence against inherited rotation, however, is the observation that none of the lower luminosity field red giants in Figure 12 have $v_{\text {rot }} \sin i$ greater than 8.5 $\mathrm{km} \mathrm{s}^{-1}$. 


\subsubsection{Induced Rotation: Stellar Companions}

As discussed earlier, three of the most luminous red giants that show significant rotation are spectroscopic binaries, and all three have short periods that could lead to orbital evolution, including tidal locking or perhaps even mass transfer. Two of the stars, $\mathrm{BD}+30^{\circ} 2034$ and $\mathrm{CD}$ $-37^{\circ} 14010$, have especially short periods and very low orbital eccentricities, which is strong evidence favoring tidal interaction and spin-up. The third star, HD 218732, also has a relatively short period, and for a stellar-mass companion, a primary radius of roughly $0.5 \mathrm{AU}$, and a semimajor axis of only a few AU, tidal interactions may well be occurring. Of the other five luminous red giants that appear to show significant rotation (including HD 3008 and BD $+22^{\circ} 2411$ ), we have not seen any signs of binary orbital motion.

If interactions with companions, stellar or substellar, can explain the high frequency of rapid rotation among luminous red giants, is this consistent with existing statistics of spectroscopic binary systems among the precursors to this evolutionary stage? In other words, what fraction of metalpoor main-sequence stars begin their evolution with companions in close enough orbits that tidal interactions will arise as the primary begins to swell in size? It does not appear that there are enough stellar companions to cause a measurable rotational velocity frequency as high as $50 \%$. We have noted already that only about $17 \%$ of field metalpoor dwarfs are spectroscopic binaries, and many of those stars have periods that are too long for tidal interactions to ever occur, even when the primary reaches a size on the order of $0.5 \mathrm{AU}$. Even granting that interactions would occur with $a \approx 1.5 \mathrm{AU}$, a combined system mass of $1.2 \mathrm{M}_{\odot}$ means an initial orbital period of about 600 days $(\log P \approx 2.8)$. Figure 5 shows that only about half the spectroscopic binaries have such short periods, so we expect that less than $10 \%$ of the main-sequence stars would eventually evolve into tidally interacting luminous red giants. Three spectroscopic binaries out of 16 stars, $19 \% \pm 11 \%$, is approximately consistent with this estimate.

\subsubsection{Induced Rotation: Planetary Companions}

Could the difference between the $\approx 10 \%$ expected and the $\approx 50 \%$ observed fractions of enhanced rotation among luminous metal-poor red giants be made up by objects whose masses are small enough that the companions of the mainsequence and subgiant stars studied by Latham et al. (2002) were undetectable? In this case tidal locking is probably not the mechanism for rotational spin-up but, rather, a merger event, whereby the secondary's orbital angular momentum is converted into rotational angular momentum of the primary.

Absorption of an orbiting planet by an evolving star is not a new idea. The rapidly rotating, apparently single giants that fall into the FK Comae class of variable stars are thought to be caused by absorption of low-mass companions (although more massive than planets), as discussed initially by Webbink (1976), Bopp \& Stencel (1981), and Livio \& Soker (1988). Eggleton (1978) and Livio (1982) suggested a mechanism for the formation of cataclysmic binaries via merger of low-mass secondaries. Peterson, Tarbell, \& Carney (1983) discovered high rotation rates among field BHB stars and noted that absorption of a planet during a star's preceding RGB stage could explain the rotation.
Soker $(1992,1996,2001)$ discussed the role absorption of planets may have in the evolution of planetary nebulae through the spin-up of AGB stars. Of special relevance to our results is the work by Siess \& Livio (1999, hereafter SL99). They discussed the spin-up of solar-mass red giant stars from the engulfment of Jovian-mass (and larger) planets as the stars expand in size. They treated the case of a rigidly rotating convective envelope, decoupled from the core. They found that planets with masses a few times that of Jupiter $\left(M_{\mathrm{J}}\right)$ can spin up such red giants to rotational velocities of a few to around $10 \mathrm{~km} \mathrm{~s}^{-1}$, depending, of course, on the mass of the planet and the radius of the star. One key point of SL99 was that the increase in the rotation rate is most pronounced for stars near the RGB tip, since that is the evolutionary stage at which the envelope's angular momentum is smallest because of the reduced envelope mass, which is the result of the retreat toward the surface of the boundary between the hydrogen-burning shell and the convective envelope.

We reexplore the absorption of a planet as a means to spin up a red giant but instead assume here that the core is coupled to the envelope. We assume that the red giant expands in radius until it engulfs a close companion of mass $M_{2}$ moving at an orbital speed of $v_{\text {orb }, 2}$. The primary radius at this point is $R_{1}$, and we ask what must be the mass of the companion to spin up the red giant primary to a rotational velocity of $v_{\text {rot, } 1}$ if all the orbital angular momentum of the secondary, $M_{2} a_{2} v_{\text {orb }, 2}$, is converted into rotational angular momentum, $I_{1} \omega_{1}$, where $a_{2}$ is the distance of the secondary from the system's center of mass. The model is very simplistic, but it gives us an idea of what sort of companion would be necessary to spin up the red giant and whether such a binary system would have been detectable when the primary star was unevolved. The major uncertainty is the moment of inertia of the primary $I_{1}$, which we parameterize as $k M R^{2}$, where $k$ is a factor (called the "radius of gyration") that must be determined from stellar evolution models. In this case one may derive a relation between the unknown ratio of the secondary to primary masses, $q \equiv M_{2} / M_{1}$, and the "known" values, the observed rotational velocity of the primary $v_{\text {rot, } 1}$ and the radius and mass of the primary, $R_{1}$ and $M_{1}$ :

$$
\frac{q^{2}}{(1+q)^{3}}=\frac{k^{2} v_{\mathrm{rot}, 1}^{2} R_{1}}{8 G M_{1}}
$$

Few estimates of moments of inertia for red giants have been published. However, Claret \& Giménez (1998) have provided such data for several stellar masses and helium contents. In the case of a star with $1 M_{\odot}$, hydrogen mass fraction $X=0.744$, and heavy-element mass fraction $Z=0.004([\mathrm{Fe} / \mathrm{H}] \approx-0.7)$, near the tip of the RGB this star reaches values comparable to those of our sample, with $\log g \approx 0.6$ and $R \approx 84 R_{\odot}$. At this point, $k \approx 0.05$. For this value and with $v_{\text {rot, } 1}=10 \mathrm{~km} \mathrm{~s}^{-1}, M_{1}=0.7 M_{\odot}$, and $R_{1}=100 R_{\odot}$, we find $q=0.0045$, so $M_{2}$ is very small, only about $5 M_{\mathrm{J}}$. In this case, the orbital period during the mainsequence stage would have been 46 days, and the velocity amplitude of the primary would have been only about 0.5 $\mathrm{km} \mathrm{s}^{-1}$ for $\sin i \approx 1$, which would be hard to detect with the facilities employed in this project. [We recall that HD 97 and HD 213467 become more interesting in this respect because their low $P\left(\chi^{2}\right)$ values imply low-amplitude velocity variability, yet their luminosities may be too low for such 
variability to be caused by velocity jitter.] Thus, it is possible that some metal-poor field stars have planetary companions in relatively close orbits, which are absorbed, along with their orbital angular momentum, late in the star's life, producing a large but rapidly rotating red giant. For constant $q$ - and $k$-values, $v_{\text {rot }} \sin i \propto R^{-1 / 2}$, so absorption of planets by stars with smaller radii would be easier to detect. Thus, our observation that high rotation only appears among the most luminous red giants in our survey hints that the putative giant-planet companions do not have smaller semimajor axes than 0.3 to $0.5 \mathrm{AU}$.

\subsection{Other Observational Consequences of Planetary Capture}

SL99 pointed out several other observational, if transient, signatures that might support the planetary merger hypothesis. Aside from the digestive aftereffect of increased rotation of the red giant, these include temporarily enhanced mass loss due to the addition of orbital energy to the red giant envelope, a possible but temporarily enhanced abundance of lithium, which will have been destroyed previously via the red giant's convective envelope, and increased rotation in the subsequent horizontal-branch stage of evolution.

\subsubsection{Enhanced Mass Loss}

SL99 returned to a model by de la Reza, Drake, \& Da Silva (1996), who postulated that deep mixing in metal-rich red giant stars could yield, temporarily, enhanced surface abundances of lithium, as well as excess flux at far-infrared wavelengths due to the formation of dust shells through the increased mass loss. The correlation between enhanced lithium abundances (first discovered by Wallerstein \& Sneden 1982) and relatively rapid surface rotation has been discussed for disk population stars by Charbonnel \& Balachandran (2000), de Medeiros et al. (2000), Reddy et al. (2002), and Drake et al. (2002). Many of these stars also show infrared flux excesses (Gregorio-Hetem et al. 1992; Gregorio-Hetem, Castilho, \& Barbuy 1993).

SL99 noted that the postulated causal connection between mixing and mass loss is unexplained but that absorption of a planet could cause both effects. The signature of the dust shell was described by de la Reza et al. (1996) as excess flux at 12, 25, and $60 \mu \mathrm{m}$, and especially the location of stars in a far-infrared color-color diagram ([25-12] vs. [60-25]). We employed the IRAS Point Source Catalog, Version 2 (1988), and the IRAS Faint Source Cata$\log$ (Moshir et al. 1992) to search for such emission from our program stars. Eleven of them were detected at $12 \mu \mathrm{m}$. (Eight of these had been discussed earlier by Smith [1998]. We also found detections for HD 83212, 111721, and 141531.) None of the stars were detected at 25 or $60 \mu \mathrm{m}$, so in no case have we found strong evidence for enhanced mass loss. Do the detections at $12 \mu \mathrm{m}$ hint at infrared excesses? Smith (1998) addressed this question by comparing $B-V$ colors with [ $V-12]$ color for a sample of field red giants. Among the metal-poor giants, he found evidence for excess flux at $12 \mu \mathrm{m}$ for only a few very luminous, variable, probably AGB, stars. But not all the luminous and variable stars showed such excesses, with TY Vir and SX Her appearing normal. In Figure 13, we show a similar figure, but here we have dereddened the $V$ magnitudes and the $b-y$ colors. The open circles represent TY Vir and SX Her. The stars we studied to construct Figure 13 include all our program stars

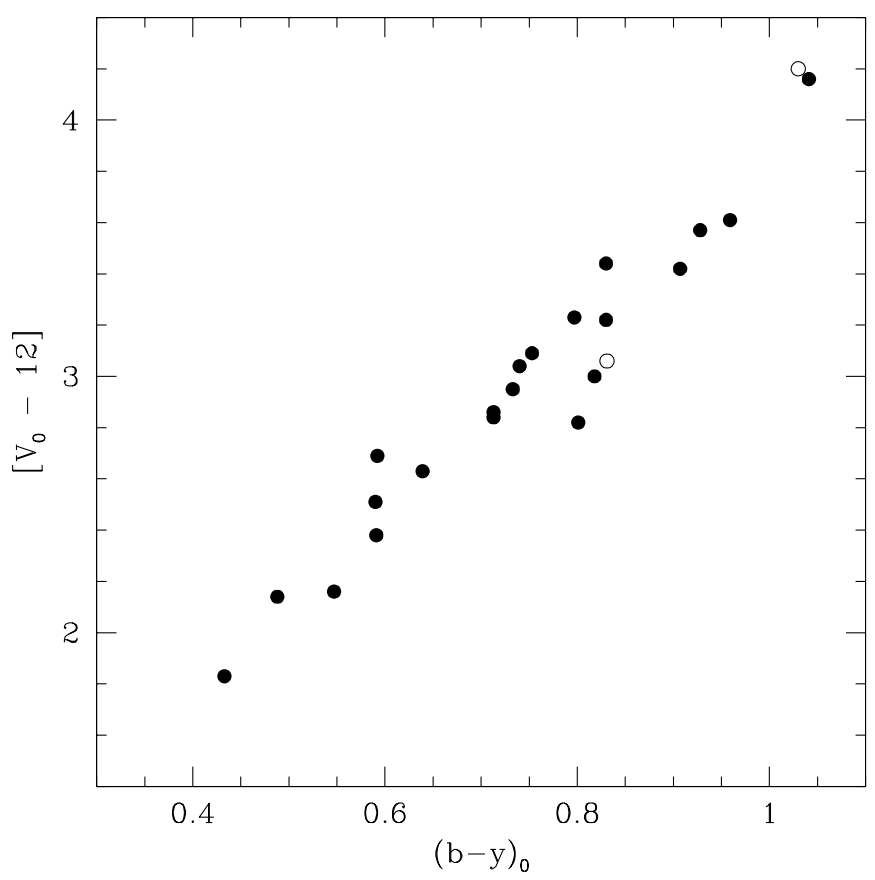

FIG. 13.- Results of a search through the IRAS Point Source Catalog for all stars in our program and in that of ATT. The $\left[V_{0}-12\right]$ colors (see Smith 1998) are compared with dereddened $b-y$ colors.

plus all the stars in ATT. No signs of excess infrared flux are seen. Three effects work against such detections among our program stars, however. First, dust shells may be brief in duration, so large initial sample sizes may be required to discover a few examples. Our sample size is smaller than those that led to the discovery of disk population red giants with measurable lithium abundances, and which were then exploited to search for excess flux in the far-infrared. Second, halo stars are rarer than disk stars and hence are, on average, more distant, so that the flux levels for dust shells around our program stars would more readily have fallen below the IRAS sensitivity limit. Finally, our program stars are, by definition, much more deficient in heavy elements than are disk population red giants, which no doubt inhibits the formation, and therefore the detection, of dust.

A common indicator of mass loss is emission or asymmetry in the line profile of the $6563 \AA$ line of $\mathrm{H} \alpha$. Smith \& Dupree (1988) reported such a study of 52 metal-poor field red giants, 24 of which are in our program. After reassigning luminosities to these stars from Table 2, we note, as did they, that the evidence for mass loss from the $\mathrm{H} \alpha$ line is confined to the most luminous stars, $M_{V} \leq-1.4$. Of the six such stars in our program observed by Smith \& Dupree, three show symmetric $\mathrm{H} \alpha$ profiles, indicating an absence of mass loss, albeit perhaps a temporary absence. However, these three stars are also among the slower rotators: HD 135148 (CH star, binary, $v_{\text {rot }} \sin i=7.8 \mathrm{~km} \mathrm{~s}^{-1}$ ), HD $141531\left(v_{\text {rot }} \sin i=7.5 \mathrm{~km} \mathrm{~s}^{-1}\right)$, and HD $221170\left(v_{\text {rot }} \sin i\right.$ $=7.4 \mathrm{~km} \mathrm{~s}^{-1}$ ). The other three luminous stars that show $\mathrm{H} \alpha$ emission, on the other hand, all show signs of both velocity jitter and high rotation (HD 3008, BD $+22^{\circ} 2411$, and HD 110281). These limited results support the idea that the rapid rotators show enhanced mass loss rates, but of course it is not clear whether the mass loss is aided by the rotation or if it arises, as suggested above, by the addition of both orbital energy and angular momentum from an accreted planet to the stellar envelope. 


\subsubsection{Lithium Abundances}

The abundance of lithium in evolved metal-poor stars is an important probe of internal mixing, as well as a possible sign of planetary ingestion. Pilachowski et al. (1993) made the first extensive study of evolved metal-poor stars. We adopt here the more recent and more extensive results from Gratton et al. (2000), who reanalyzed the earlier results from Pilachowski et al. (1993) to minimize small systematic differences. On a scale in which the logarithm of the number density of hydrogen, $\log \epsilon(\mathrm{H})$, is defined to be 12.00 , they found that metal-poor main-sequence and turnoff stars show $\log \epsilon(\mathrm{Li})=2.4$. We discuss here only their results for our program stars, which are summarized in Figure 14. Arrows refer to upper limits. The figure shows that as the metal-poor stars evolve to higher luminosities, the lithium abundances drop, becoming essentially undetectable by $M_{V} \approx-0.7$, with the interesting exception of HD 118055, at $M_{V} \approx-1.5$ (although its lithium abundance is very low). No other high-luminosity stars have lithium detections, either those in our program or the other such stars studied by Gratton et al. (2000). Two additional points deserve comment. First, Figure 14 does not include the RHB stars. None of the RHB stars analyzed by Gratton et al. (2000) had detectable lithium lines. This included three of the RHB stars in our program, HD 20, 108317, and 110885. Second, none of the metal-poor, luminous red giants that we have found to have $v_{\text {rot }} \sin i>8.5 \mathrm{~km} \mathrm{~s}^{-1}$ (BD +30 2034, HD 110281, HD 121135, BD + $8^{\circ} 2856, \mathrm{CD}-37^{\circ} 14010, \mathrm{HD}$ 214925 , and HD 218732) or the two stars with implied $v_{\text {rot }}$ values exceeding $12 \mathrm{~km} \mathrm{~s}^{-1}$, HD 3008 and BD +22 2411 , were studied by Pilachowski et al. (1993) or by Gratton et al. (2000). Over the past 5 years, we have acquired the necessary high-resolution, high-S/N echelle spectra using the KPNO $4 \mathrm{~m}$ telescope for all the above stars, except HD 121135. Detailed abundance analyses will be presented

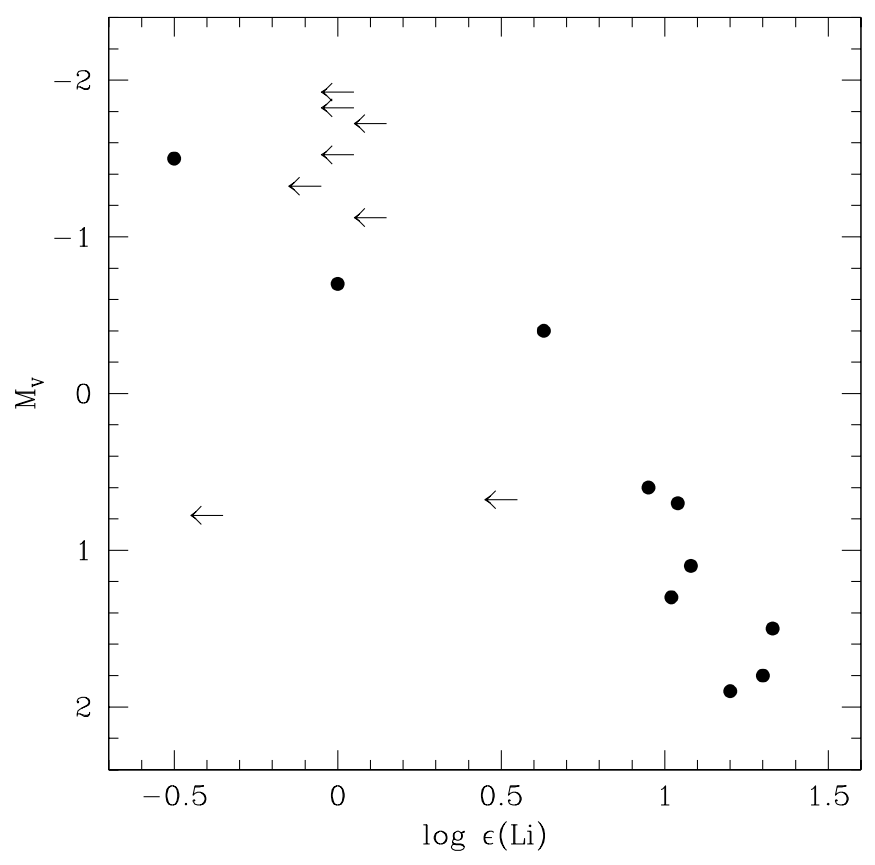

FIG. 14.-Measured abundances of lithium in our program stars, or upper limits thereof, from Gratton et al. (2000). later, but in no case was the lithium line strong (or even detectable, despite typical $\mathrm{S} / \mathrm{N}$ levels of up to 300 pixel $^{-1}$ ).

It is worth recalling, however, that lithium enhancements have been found in a few evolved stars in globular clusters. All these stars have high luminosities and, also, large radii (or recently did so). The 25.7 day Cepheid variable V42 in M5 (Carney, Fry, \& Gonzalez 1998) is probably a postAGB star on its way across the Hertzsprung-Russell diagram, yet it has $\log \epsilon(\mathrm{Li})=+1.8$. Smith, Shetrone, \& Keane (1999) found that the 105 day variable V2 located near the tip of the RGB in NGC $362\left(M_{V} \approx-2.2\right)$ is enhanced in lithium, with $\log \epsilon(\mathrm{Li})=+1.2$. Smith et al. (1999) noted in particular that the source of the lithium could have been due to the ingestion of a planet with a mass of roughly $4 M_{\mathrm{J}}$. Finally, Kraft et al. (1999) found that the luminous firstascent RGB star IV-101 in M3 has an extreme enhancement of lithium, $\log \epsilon(\mathrm{Li})=+3.0$. This is the least evolved of the three known lithium-rich giants in globular clusters but still has $M_{V}=-1.9$ and a radius exceeding $50 R_{\odot}$. No rotational velocities have been reported for any of these three stars.

\subsection{Counterevidence?}

Are planets with masses of a few times that of Jupiter in orbit around metal-poor field stars consistent with what is known currently about the existence of planets among Population I and II systems? Gonzalez et al. (2001) and Reid (2002) have explored the question of the frequency of planetary companions compared with the metallicity of the parent stars, and, at least near solar metallicities, shortperiod planetary systems appear to be more common around more metal-rich stars. (We find it nonetheless interesting that the first star to be found with a planetary or brown dwarf companion was HD 114762, a well-known metal-poor dwarf; Latham et al. 1989.) The conclusions from solar-metallicity stars should not, however, be extrapolated all the way to the metal-poor domain, especially if more than one mode of planetary formation exists. Only when a search for planets around very metal-poor stars has been completed would we be able to conclude anything about the applicability of the results from Gonzalez et al. (2001) and Reid (2002) to the very metal-poor stars.

In this vein, we note that a search for transits of mainsequence stars in the metal-poor $([\mathrm{Fe} / \mathrm{H}] \approx-0.7)$ globular cluster 47 Tuc failed to find any evidence of "hot Jupiters" (Gilliland et al. 2000), which appears to support the idea that planets are rare around metal-poor stars. For two reasons, we believe that these results do not contradict our conclusions.

First, the Gilliland et al. (2000) experiment only lasted about a week and thus was not sensitive to planets with much longer periods, as would be required by our result that excess rotation does not appear among field metal-poor giants until they have swollen to relatively large sizes, $40 R_{\odot}$ or larger $\left(M_{V}<-1.5\right)$. The probability that a planetary orbit is properly aligned to produce a transit is proportional to the radius of the star divided by the semimajor axis of the orbit and, thus, goes down with increasing period as $P^{-2 / 3}$. Systems with periods of, say, 50 days are thus about 5 times less likely to be detected than those with periods of 4 days or so, had the observations covered such a long period. However, the observations covered a period of only about a week, and the chance of such observations' discovering a wide system with an orbital period of months or longer 
diminishes with the ratio of the duration of the observations to the orbital period. The probability that Gilliland et al. would have found a system with a period of 50 days is therefore reduced by an additional factor of about 7 . Gilliland et al. estimated that they should have detected transits by 17 "hot Jupiters" if the frequency in 47 Tuc was the same as has been observed for solar-type stars in the solar neighborhood. According to the arguments that we have just presented, this estimate should be reduced by about a factor of 35 for orbital periods of 50 days, and even more for longer periods.

Second, 47 Tuc appears, like many other globular clusters, to be somewhat deficient in stellar spectroscopic binary systems (Côté et al. 1996). If the low frequency is caused by a process that impedes the formation of stellar binary systems, perhaps that same process impedes the formation of planetary systems. More likely, the cause of the spectroscopic binary deficiency is dynamical, involving disruption of existing systems, in which case planetary systems, with lower gravitational binding energies, would be more easily disrupted. To disentangle the role of dynamical interactions from the role of low metallicity, it is important to search for planets orbiting metal-poor field stars, where the dynamical effects should be minimized, or, at least, in clusters where dynamical evolution has been minimal.

We have speculated that up to one-third of the most luminous field halo red giants acquire their enhanced rotational velocities through absorbing a planetary companion. The implied planetary frequency is considerably higher than that determined for local disk population dwarfs, in which roughly $5 \%$ of the stars have planetary companions with orbital periods of 1500 days or less (Zucker \& Mazeh 2001). One must wonder whether the halo and disk populations' fractions of planetary companions are so different or if we have overestimated the degree or the cause of rotational broadening that we have derived for the luminous metalpoor giants.

\subsection{Transport of Internal Angular Momentum?}

The detection of rapid rotation among BHB stars was a surprise because metal-poor main-sequence stars had not been found to show detectable rotation, as discussed above. As Peterson et al. (1983) discussed, either the rapid rotation was acquired or the angular momentum had been confined below the surface layers of the main-sequence antecedents and exposed by surface mass loss or by angular momentum transport. Peterson et al. (1995) explored possible connections between rotation among cluster BHB stars and deep mixing that might be manifested by altered abundances of oxygen, but they found no clear relationships.

Our results may help rule out enhanced red giant rotation due to transport of internal angular momentum. We assume that the transport of internal angular momentum to the surface is accomplished via the deep convection zones in the cool red giants. However, the convection zone is not at its deepest when the star is near the tip of the RGB. Its maximum depth of penetration occurs at an earlier stage and is believed to be the explanation of the "bump" observed in the luminosity functions of globular cluster systems (Thomas 1967; Iben 1968; Renzini \& Fusi Pecci 1988). This "bump" is seen at $V$-band luminosities about 0.2 to 0.3 mag brighter than the horizontal branch (Fusi Pecci et al. 1990; Lee \& Carney 1999). If internal angular momentum is transported to the surface by convection, it should begin to appear at $M_{V}$ levels of +1 to 0 mag. Figure 12 shows that this is not observed. The six stars at this luminosity with high rotation rates are all hotter than the RGB and are clearly RHB stars, successors to, rather than predecessors of, the luminous RGB state. In fact, were the rapid rotation to appear at this magnitude level, it should be at higher velocities than we have found in the luminous red giants because the RGB luminosity function bump appears among stars with radii of about $10 R_{\odot}$ and smaller, compared with the $40 R_{\odot}$ and larger that apply to stars with $M_{V} \leq-1.5$. Thus, it appears that transport of internal angular momentum is unlikely to be the explanation for the high rotational velocities seen among the luminous red giants and the RHB stars in our sample.

\section{ROTATION OF RED HORIZONTAL-BRANCH STARS}

Following ignition of helium in its core, a metal-poor low-mass red giant rapidly evolves into the horizontalbranch stage of evolution. If rotation is relatively common among red giants nearing the end of their shell hydrogen burning evolution, is it equally common among field horizontal-branch stars?

Since the discovery work by Peterson et al. (1983), relatively rapid rotation has been found among a significant fraction of globular cluster BHB stars (see Peterson 1985a, 1985b; Peterson et al. 1995; Cohen \& McCarthy 1997; Behr, Cohen, \& McCarthy 2000a; Behr et al. 2000b; Recio-Blanco et al. 2002). Soker (1998) and SL99 discussed the possibility that the absorption of a planet by a large-radius red giant might explain not only the rapid rotation seen among some BHB stars, but indeed also how some BHB stars are produced. Metallicity is often described as the "first parameter," in that metal-poor clusters tend to have a higher frequency of their horizontal-branch stars bluer than the instability strip. But some clusters with similar metallicities have very different distributions of stars along the horizontal branch, and for that matter, some individual clusters have an unusually wide distribution of stars across the horizontal branch despite having uniform metallicities. This phenomenon is often called the "second-parameter problem." What else, besides metallicity, can help remove envelope mass such that a star winds up on the BHB, compared with the more massive envelope stars that lie on the RHB? Soker (1998) argued that the absorption of a planet's orbital energy would lead to enhanced mass loss, thereby preferentially forming stars with less massive envelopes: BHB stars. The calculations of SL99 support this conjecture, although accurate predictions are difficult.

Before testing this model with our results, we must first ask whether field BHB stars show the same behavior as found among cluster stars. Kinman et al. (2000) have made the most comprehensive study of field BHB stars, finding that they behave similarly to such stars found in globular clusters. Briefly, rotational velocities of up to $40 \mathrm{~km} \mathrm{~s}^{-1}$ have been found, and there is some evidence that, despite their smaller radii, the hottest BHB stars rotate more slowly than do their cooler counterparts. Could the higher rotational velocities have originated via stellar binary system interactions for some stars or via planetary absorption during the primary's red giant stage for other stars? 
One interesting test, although qualitative at this point, is to ask whether red horizontal-branch stars rotate. If not, then rotation and mass loss may indeed be coupled, since the RHB stars in general should have lost less mass. If RHB stars do rotate, then the issue is not so simple.

It is now clear that a significant fraction of the field RHB stars rotate at detectable levels. HD 195636 was found by Preston (1997) to have significant line broadening, most likely due to rotation, with $v_{\text {rot }} \sin i \approx 25 \mathrm{~km} \mathrm{~s}^{-1}$. We confirm that the star is broad-lined, with $v_{\text {rot }} \sin i \approx 22 \mathrm{~km} \mathrm{~s}^{-1}$. Five other RHB stars in our sample also have significant rotational broadening: $\mathrm{CD}-23^{\circ} 72\left(9 \mathrm{~km} \mathrm{~s}^{-1}\right)$, HD 82590 $\left(13 \mathrm{~km} \mathrm{~s}^{-1}\right)$, HD $119516\left(9 \mathrm{~km} \mathrm{~s}^{-1}\right)$, HD $184266\left(12 \mathrm{~km} \mathrm{~s}^{-1}\right)$, and HD $214362\left(11 \mathrm{~km} \mathrm{~s}^{-1}\right)$. Thus, at least six of the 15 stars we identified as RHB stars in Figure $1(40 \%)$ rotate at velocities comparable to or greater than our instrumental resolution.

What fraction of BHB stars show significant rotation? Figure 15 shows the fractional distribution of rotational velocities derived by Kinman et al. (2000) for field stars, and by Peterson et al. (1995) and Cohen \& McCarthy (1997) for cluster stars. The spectral resolving powers for these programs differed $(15,000,20,000$, and 34,000, respectively), so assessing what fraction of BHB stars show elevated rotational velocities is somewhat difficult. But adopting a value of $v_{\text {rot }} \sin i>15 \mathrm{~km} \mathrm{~s}^{-1}$ for the cluster stars and $v_{\text {rot }} \sin i>20 \mathrm{~km} \mathrm{~s}^{-1}$ for the field stars, the fractions are $19 / 72$ and $6 / 26$, or $26 \%$ and $23 \%$, respectively. These are comparable to the fraction derived above for the field RHB stars.

Qualitatively, then, we confirm that a significant fraction of both the BHB and RHB stars, like the luminous red giants, are rotating. Taken at face value, this implies that rotation alone does not determine whether an evolving red giant will ultimately wind up on the blue or the red side of the horizontal branch. The fractions of enhanced rotation among the BHB stars may be higher, of course, since we

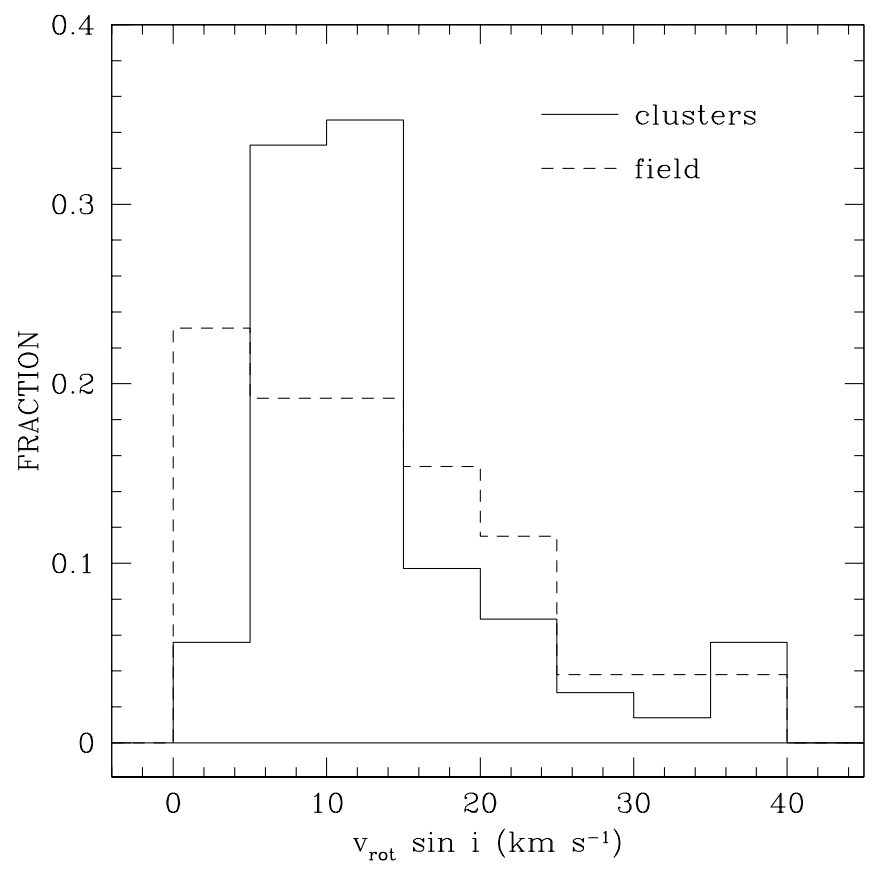

FIG. 15.-Fractional distribution of rotational velocities of field BHB stars (Kinman et al. 2000) and cluster BHB stars (Peterson et al. 1995; Cohen \& McCarthy 1997). have adopted rather conservative limits. Indeed, the cluster $\mathrm{BHB}$, field $\mathrm{BHB}$, and field RHB stars may all have fractions of enhanced rotation consistent with the $40 \%$ to $50 \%$ fraction that we derive for the luminous field red giants (which includes both the tidal spin-up from stellar companions and the possible absorption of planetary companions discussed above). We suggest that the origin of the elevated surface rotation of horizontal-branch stars arises from spin-up during their earlier evolutionary stage as large, luminous, red giants.

What does this mean for the origin of the second parameter? There are two significant complications in exploring this issue. First, rotation may be important in generating BHB stars, as suggested by Soker (1998) and by SL99, but if so, the effect of rotation on the removal of a red giant's envelope to create a BHB descendent is more complex than envisioned, since RHB stars appear to have about the same amount of surface angular momentum as do BHB stars. Consider the maximum rotation values, whereby we may be able to reduce the effect of the unknown inclinations of the rotational axes to our line of sight. Among both field and cluster BHB stars, the maximum rotational values are about $40 \mathrm{~km} \mathrm{~s}^{-1}$. This is roughly consistent with those of the RHB stars $\left(\approx 22 \mathrm{~km} \mathrm{~s}^{-1}\right)$, given that the BHB stars have radii ranging between 3 and $4 R_{\odot}$, while the RHB stars have radii about twice as large, from 5 to $9 R_{\odot}$. (We recognize that our assumptions here are simplistic, and that more detailed predictions involving internal mass and rotation distributions, such as those of Sills \& Pinsonneault [2000], will be required.) So we find that the percentage of RHB stars that have detectable rotational broadening is the same as for BHB stars, and the maximum values derived for $v_{\text {rot }} \sin i$ times the estimated stellar radii are comparable. We add one point to the discussion, whose relevance is compromised by small number statistics. We note that among the 15 or so RHB stars in our sample, there is only one stellar binary system: HD 108317, which must have a period of over $10 \mathrm{yr}$, and the star's estimated $v_{\text {rot }} \sin i$ value is modest, only $5 \mathrm{~km} \mathrm{~s}^{-1}$ (or less). Thus, in no case is the rotation of the RHB stars in our sample attributable to an existing stellar companion.

A second and more important problem in coupling the second-parameter problem to spin-up of luminous RGB stars is that the second parameter is defined with respect to globular clusters. Stellar binaries, and especially planetary systems, are highly vulnerable to disruption in dense cluster environments. However, rapid rotation is nonetheless seen in cluster BHB stars, so surveys of rotation among cluster RHB stars might be very revealing. Just to make the comparisons even more complex, the average metallicity, $\langle[\mathrm{Fe} / \mathrm{H}]\rangle$, of the RHB stars in our program is -1.9 , a point at which few globular clusters have significant numbers of RHB stars, thanks to the first parameter.

There are two additional puzzles that appear upon more careful consideration. First, it appears that considerable angular momentum is lost during the red giant stage (or at its termination). The large radii of the luminous, rotating red giants in our sample, 50 to $100 R_{\odot}$, are from 10 to 30 times larger than those of RHB and BHB stars. However, the maximum rotational velocities of the red giants are comparable to those of the field RHB stars. Whatever causes rotation, it appears that surface angular momentum is not conserved as a star moves from the tip of the RGB to the horizontal branch. Mass loss during the latest stages of red 
giant evolution, or during the transition from the red giant to the horizontal-branch stage, could contribute to the loss of surface angular momentum.

Second, and equally puzzling, is that field metal-poor RR Lyrae variables show no signs of rotation at all (Clementini et al. 1995; Peterson, Carney, \& Latham 1996; Lambert et al. 1996; Fernley \& Barnes 1996). Could pulsation somehow contribute to mass loss that expels surface angular momentum? We do not have answers to these puzzles, but our data indicate clearly that the issue of the evolution of surface (and presumably internal) angular momentum in evolving low-mass stars is much more complex than traditional models of stellar evolution assume.

\section{FUTURE WORK}

Our speculations concerning rotation among luminous metal-poor red giants and RHB stars and its causes will, we hope, spur several new observational efforts.

First, we encourage observations of our program stars with higher resolution spectroscopy to confirm (or dismiss) our estimated rotational velocities. The work should be extended to a larger - perhaps much larger - sample of field metal-poor red giants and RHB stars. Similar studies of metal-rich red giants should also prove rewarding, as, perhaps, might searches for rotational broadening among RR Lyrae variables. High-resolution, high-S/N spectra of these stars, especially those showing rotation and possible planetary absorption, should be obtained to see whether lithium abundances are elevated. Inspection of the $\mathrm{H} \alpha$ line profiles to explore the possible presence of mass loss would also be very useful.

Second, similar analyses of globular cluster giants and RHB stars should be undertaken using existing and new spectra. The effects of environment on binary characteristics, stellar rotation, and lithium abundances would be very revealing. If the rotation seen among luminous metal-poor field red giants is due to the acquisition of planetary orbital angular momentum, and if planetary systems are rare in globular clusters, then luminous globular cluster giants should not show excess rotation, unless spun up by close stellar companions. It would, of course, be interesting to study rotation among RHB stars in those clusters where the BHB stars have been found to rotate significantly. Pilachowski et al. (2000) have published a survey of $261 \mathrm{red}$ giants in globular clusters in a search for elevated lithium abundances, with no new discoveries.

Third, we suggest that the idea that planets form only, or at least preferentially, around the most metal-rich stars should be questioned. In particular, we encourage a thorough spectroscopic search for Jovian-mass planets orbiting metal-poor main-sequence and lower giant branch stars, especially those not known to be spectroscopic binaries. For example, a system containing a $0.7 M_{\odot}$ star and a planet with a mass equal to that of Jupiter in an orbit of 0.5 or 2.0 AU would produce a reflex motion of 85 or $25 \mathrm{~m} \mathrm{~s}^{-1}$, respectively, for $\sin i=1.0$. Existing instrumentation could detect such motions, if the observing program was sustained for a period of several years.

Finally, we recommend further work to study the possibilities that starspots cause velocity jitter, especially in the cases of HD 3008 and $\mathrm{BD}+22^{\circ} 2411$. High-resolution and high-S/N spectroscopy might reveal such motions via line bisector analyses or Doppler imaging, and photo- metric or spectroscopic monitoring of the $\mathrm{Ca}$ II $\mathrm{K}$ line might be revealing.

\section{SUMMARY}

We have been monitoring the radial velocities of 91 metal-poor field red giants for time spans of up to 18 years. We have derived spectroscopic orbits for 14 binary stars, and we see clear signs of orbital motion in two others: BD $-1^{\circ} 2582$ and HD 108317. Both CH stars in our sample, BD $-1^{\circ} 2582$ and HD 135148, are spectroscopic binaries.

The spectroscopic binary fraction for stars with $[\mathrm{Fe} / \mathrm{H}]$ $\leq-1.4$ and $P<6000$ days, $16 \% \pm 4 \%$, is very similar to the binary frequency of dwarfs in the same metallicity and period regime, $17 \% \pm 2 \%$. We conclude that there have been few stellar mergers among our entire sample of stars, which range from $M_{V}=+2.0$ to $M_{V}=-2.8$. But the distributions of the orbital periods and the giants differ, even when the sample is restricted to stars with orbital periods exceeding 20 days, the regime in which orbital evolution of dwarfs has been recognized. It appears that a small fraction of the red giants in our sample have undergone additional orbital evolution, leading to longer periods and more circular orbits.

We detect velocity jitter among the most luminous red giants. About $40 \%$ of the program stars with $M_{V} \leq-1.4$ have large ratios of external to internal errors and very small $P\left(\chi^{2}\right)$ values. The two best-observed cases, HD 3008 and $\mathrm{BD}+22^{\circ} 2411$, show apparent pseudoperiodicities of 172 and 186 days, longer than any known red giant pulsators in globular clusters. Because the periods are not robust, orbital motion is probably also ruled out. This leaves us with rotational modulation as the leading candidate for the source of the observed velocity jitter. Photometric variability seen in four of the stars with velocity jitter supports this idea, involving some form of time-variable bright or dark regions on the stars' surfaces. A simple single-starspot model was not successful in explaining the velocity and photometric behaviors of HD 3008, but we have hardly exhausted the options.

The implied rotational velocities for HD 3008 and BD $+22^{\circ} 2411$, both of which have estimated radii near $50 R_{\odot}$, are 14.5 and $12.7 \mathrm{~km} \mathrm{~s}^{-1}$, respectively. Matching a large grid of synthetic spectra to the spectra of all our program stars shows that almost half the highly evolved red giants, with $M_{V} \leq-1.4$, have $v_{\text {rot }} \sin i$ values that exceed our instrumental resolution of $8.5 \mathrm{~km} \mathrm{~s}^{-1}$. In three cases, BD $+30^{\circ} 2034$, $\mathrm{CD}-37^{\circ} 14010$, and HD 218732, this may be attributed to tidal locking with orbiting stellar companions, based on low orbital eccentricities, low ratios of the estimated companion separation to the estimated red giant radii, or both. Nonetheless, there appear to be too few spectroscopic binary systems with short enough periods among field metal-poor main-sequence stars to result in strong tidal interactions that could explain such a large frequency of high rotational velocities among field metal-poor, luminous red giants. An alternative explanation is absorption of large planetary companions in close orbits ( $a \approx 0.5 \mathrm{AU})$. Transferal of orbital angular momentum from a planet with $M \approx 5 M_{\mathrm{J}}$ would spin up a red giant with a radius of $100 R_{\odot}$ to rotational velocities consistent with what we have found to be common among the largest stars in our survey. We argue against the idea that the luminous red giant rotation is due to transport of internal angular momentum to the surface. Were this the 
case, we would expect to see the onset of surface rotation at roughly the evolutionary stage when the convection zone is at its deepest, $M_{V} \approx 0-1 \mathrm{mag}$, which is not seen in our data.

About $40 \%$ of the field RHB stars in our sample also show detectable rotational velocities. This strongly suggests that the long-known incidence of unexpectedly high rotation among field and cluster BHB stars is caused originally by rotational spin-up during their earlier evolution as luminous red giants. The similar prevalence and degree of rotational angular momentum found among RHB and BHB stars illustrates that the "second parameter" may not be directly related to the presence or absence of rotation. The maximum rotational velocities of the field RHB stars are comparable to those seen in field and cluster BHB stars, when allowance is made for the larger radii of the RHB stars. However, neither the BHB nor the RHB stars' rotational velocities are consistent with spin-up and simple conservation of angular momentum of the rotating luminous red giants in our sample. As a final puzzle, we note that field RR Lyrae variables do not appear to have any rotation at the level of detectability in our sample. Perhaps pulsation is able to enhance the loss of mass and angular momentum.

We thank R. Davis for many years of service maintaining the database for the CfA Digital Speedometers, and J. Caruso, J. Zajac, P. Berlind, and G. Torres for making many of the observations. We thank G. Torres in particular for helpful discussions of the starspot model. B. W. C. thanks the National Science Foundation for grants AST 96-19881 and AST 99-88156 to the University of North Carolina. J. B. L. thanks the National Science Foundation for grants AST 96-19628 and AST 99-88247 to Bowling Green State University. We especially acknowledge the great utility of the SIMBAD database, maintained by the CDS in Strasbourg, France.
Alonso, A., Arribas, S., \& Martínez Roger, C. 1994, A\&AS, 107, 365 1995, A\&A, 297, 197

1998, A\&AS, 131, 209

1999, A\&AS, 140, 261 (erratum 376, 1039 [2001])

Anthony-Twarog, B. J., \& Twarog, B. A. 1994, AJ, 107, 1577 (ATT) .1998, AJ, 116, 1922

Ardeberg, A., \& Lindgren, H. 1991, A\&A, 244, 310

Arribas, S., \& Martínez Roger, C. 1987, A\&AS, 70, 303

Barnes, T. G., III, Moffett, T. J., \& Slovak, M. H. 1987, ApJS, 65, 307

Behr, B. B., Cohen, J. G., \& McCarthy, J. K. 2000 a, ApJ, 531, L37

Behr, B. B., Djorgovski, S. G., Cohen, J. G., McCarthy, J. K., Côté, P.,

Piotto, G., \& Zoccali, M. 2000b, ApJ, 528, 849

Bessell, M. S., \& Norris, J. 1984, ApJ, 285, 622

Bond, H. E. 1980, ApJS, 44, 517

Bopp, B. W., \& Stencel, R. E. 1981, ApJ, 247, L131

Brown, J. A., \& Wallerstein, G. 1992, AJ, 104, 1818

Burstein, D., \& Heiles, C. 1982, AJ, 87, 1165

Busso, M., Gallino, R., Lambert, D. L., Travaglio, C., \& Smith, V. V. 2001, ApJ, 557, 802

Carney, B. W. 1980, AJ, 85, 38 1983, AJ, 88, 623

Carney, B. W., Fry, A. M., \& Gonzalez, G. 1998, AJ, 116, 2984

Carney, B. W., \& Latham, D. W. 1986, AJ, 92, 60 (CL86)

Carney, B. W., Latham, D. W., \& Laird, J. B., \& Aguilar, L. A. 1994, AJ, 107,2240

Carney, B. W., Latham, D. W., Laird, J. B., Grant, C. E., \& Morse, J. A. 2001, AJ, 122, 3419

Charbonnel, C., \& Balachandran, S. C. 2000, A\&A, 359, 563

Claret, A., \& Giménez, A. 1998, A\&AS, 133, 123

Clementini, G., Carretta, E., Gratton, R., Merighi, R., Mould, J. R., \& McCarthy, J. K. 1995, AJ, 110, 2319

Cohen, J. G., \& McCarthy, J. K. 1997, AJ, 113, 1353

Côté, P., Pryor, C., McClure, R. D., Fletcher, J. M., \& Hesser, J. E. 1996, AJ, 112,574

de la Reza, R., Drake, N. A., \& da Silva, L. 1996, ApJ, 456, L115

de Medeiros, J. R., do Nascimento, J. D., Jr., Sankarankutty, S., Costa, J. M., \& Maia, M. R. G. 2000, A\&A, 363, 239

Drake, N. A., de la Reza, R., da Silva, L., \& Lambert, D. L. 2002, AJ, 123 , 2703

Duquennoy, A., \& Mayor, M. 1991, A\&A, 248, 485

Eggleton, P. P. 1978, Sci. Today (Bombay), 13(s.n.), 22

Fernie, J. D. 1983, PASP, 95, 782

Fernley, J., \& Barnes, T. G. 1996, A\&A, 312, 957

Fernley, J. A., Lynas-Gray, A. E., Skillen, I., Jameson, R. F., Marang, F., Kilkenny, D., \& Longmore, A. J. 1989, MNRAS, 236, 447

Fusi Pecci, F., Ferraro, F. R., Crocker, D. A., Rood, R. T., \& Buonanno, R. 1990, A\&A, 238, 95

Gilliland, R. L., et al. 2000, ApJ, 545, L47

Goldberg, D., Mazeh, T., Latham, D. W., Stefanik, R. P., Carney, B. W., \& Laird, J. B. 2002, AJ, 124, 1132

Gonzalez, G., Laws, C., Tyagi, S., \& Reddy, B. E. 2001, AJ, 121, 432

Gratton, R., Quarta, M. L., \& Ortolani, S. 1986, A\&A, 169, 208

Gratton, R. G., Sneden, C., Carretta, E., \& Bragaglia, A. 2000, A\&A, 354 169

Gregorio-Hetem, J., Castilho, B. V., \& Barbuy, B. 1993, A\&A, 268, L25

Gregorio-Hetem, J., Lépine, J. R. D., Quast, G. R., Torres, C. A. O., \& de la Reza, R. 1992, AJ, 103, 549

Griffin, R. F. 1980, Observatory, 100, 193

Gunn, J. E., \& Griffin, R. F. 1979, AJ, 84, 752

\section{REFERENCES}

Hanson, R. B., Sneden, C., Kraft, R. P., \& Fulbright, J. 1998, AJ, 116, 1286 Hinkle, K. H., Scharlach, W. W. G., \& Hall, D. N. B. 1984, ApJS, 56, 1

Hoffmeister, C., Richter, G., \& Wenzel, W. 1985, Variable Stars (Berlin: Springer)

Hut, P., et al. 1992, PASP, 104, 981

Iben, I., Jr. 1968, Nature, 220, 143

IRAS Point Source Catalog, Version 2. 1988, Joint IRAS Science Working Group (Washington: GPO)

Ivans, I. I., Kraft, R. P., Sneden, C., Smith, G. H., Rich, R. M., \& Shetrone, M. 2001, AJ, 122, 1438

Ivans, I. I., Sneden, C., Kraft, R. P., Suntzeff, N. B., Smith, V. V., Langer, G. E., \& Fulbright, J. P. 1999, AJ, 118, 1273

Jasniewicz, G., \& Mayor, M. 1986, A\&A, 170, 55

1988, A\&A, 203, 329

Jensen, K. S., \& Olsen, E. H. 1979, PASP, 91, 664

Jones, R. V., Carney, B. W., \& Latham, D. W. 1988, ApJ, 332, 206

Joy, A. H. 1954, ApJS, 1, 39

Kinman, T., Castelli, F., Cacciari, C., Bragaglia, A., Harmer, D., \& Valdes, F. 2000, A\&A, 364, 102

Kraft, R. P. 1994, PASP, 106, 553

Kraft, R. P., Peterson, R. C., Guhathakurta, P., Sneden, C., Fulbright, J. P., \& Langer, G. E. 1999, ApJ, 518, L53

Kraft, R. P., Sneden, C., Langer, G. E., \& Prosser, C. F. 1992, AJ, 104, 645

Kraft, R. P., Sneden, C., Langer, G. E., \& Shetrone, M. D. 1993, AJ, 106 1490

Kraft, R. P., Sneden, C., Langer, G. E., Shetrone, M. D., \& Bolte, M. 1995 AJ, 109, 2586

Kraft, R. P., Sneden, C., Smith, G. H., Shetrone, M. D., \& Fulbright, J. 1998, AJ, 115, 1500

Kraft, R. P., Sneden, C., Smith, G. H., Shetrone, M. D., Langer, G. E., \& Pilachowski, C. A. 1997, AJ, 113, 279

Kurtz, M. J., \& Mink, D. J. 1998, PASP, 110, 934

Kurucz, R. L. 1992, in IAU Symp. 149, The Stellar Populations of Galaxies, ed. B. Barbuy \& A. Renzini (Dordrecht: Kluwer), 225

Kurucz, R. L., Furenlid, I., Brault, J., \& Testerman, L. 1984, Solar Flux Atlas from 296 to $1300 \mathrm{~nm}$ (Sunspot, NM: Natl. Sol. Obs.)

Lambert, D. L., Heath, J. E., Lemke, M., \& Drake, J. 1996, ApJS, 103, 183

Latham, D. W. 1985, in IAU Colloq. 88, Stellar Radial Velocities, ed. A. G. D. Philip \& D. W. Latham (Schenectady: L. Davis), 5

. 1992, in IAU Colloq. 135, Complementary Approaches to Double and Multiple Star Research, ed. H. A. McAlister \& W. L. Hartkopf (ASP Conf. Ser. 32) (San Francisco: ASP), 110

Latham, D. W., Mazeh, T., Carney, B. W., McCrosky, R. E., Stefanik, R. P., \& Davis, R. J. 1988, AJ, 96, 567

Latham, D. W., et al. 1992, AJ, 104, 774

Latham, D. W., Mazeh, T., Stefanik, R. P., Mayor, M., \& Burki, G. 1989 Nature, 339, 38

Latham, D. W., Stefanik, R. P., Torres, G., Davis, R. J., Mazeh, T., Carney, B. W., Laird, J. B., \& Morse, J. A. 2002, AJ, 124, 1144

Lee, J.-W., \& Carney, B. W. 1999, AJ, 118, 1373

Leep, E. M., \& Wallerstein, G. 1981, MNRAS, 196, 543

Liu, T., \& Janes, K. A. 1990, ApJ, 354, 273

Livio, M. 1982, A\&A, 112, 190

Livio, M., \& Soker, N. 1988, ApJ, 329, 764

Luck, R. E., \& Bond, H. E. 1985, ApJ, 292, 559

Lupton, R. H., Gunn, J. E., \& Griffin, R. F. 1987, AJ, 93, 1114

Mathieu, R. D., Duquennoy, A., Latham, D. W., Mayor, M., Mermilliod, J.-C., \& Mazeh, T. 1992, in Binaries as Tracers of Stellar Formation, ed. A. Duquennoy \& M. Mayor (Cambridge: Cambridge Univ. Press), 278 
Mayor, M., \& Mermilliod, J.-C. 1984, in IAU Symp. 105, Observational Tests of Stellar Evolution Theory, ed. A. Maeder \& A. Renzini (Dordrecht: Reidel), 411

Mayor, M., et al. 1997, AJ, 114, 1087

McClure, R. D. 1997, PASP, 109, 536

McClure, R. D., \& Woodsworth, A. W. 1990, ApJ, 352, 709

Menzies, J. W., Marang, F., Laing, J. D., Coulson, I. M., \& Engelbrecht, C. A. 1991, MNRAS, 248, 642

Moffett, T. J., \& Barnes, T. G., III. 1985, ApJS, 58, 843 1987, PASP, 99, 1206

Moshir, M., et al. 1992, Explanatory Supplement to the IRAS Faint Source Survey, Version 2 (Pasadena: JPL)

Nordström, B., Latham, D. W., Morse, J. A., Milone, A. A. E., Kurucz,

R. L., Andersen, J., \& Stefanik, R. P. 1994, A\&A, 287, 338

Norris, J., Bessell, M. S., \& Pickles, A. J. 1985, ApJS, 58, 463 (NBP)

Norris, J. E., \& Da Costa, G. S. 1995, ApJ, 447, 680

Norris, J. E., Peterson, R. C., \& Beers, T. C. 1993, ApJ, 415, 797

Paczyński, B. 1971, ARA\&A, 9, 183

Parsons, S. B., \& Ake, T. B. 1998, ApJS, 119, 83

Peterson, R. C. 1985a, ApJ, 289, 320 1985b, ApJ, 294, L35

Peterson, R. C., Carney, B. W., \& Latham, D. W. 1996, ApJ, 465, L47

Peterson, R. C., Kurucz, R. L., \& Carney, B. W. 1990, ApJ, 350, 173

Peterson, R. C., Rood, R. T. \& Crocker, D. A. 1995, ApJ, 453, 214

Peterson, R. C., Tarbell, T. D., \& Carney, B. W. 1983, ApJ, 265, 972

Pilachowski, C. A., Sneden, C., \& Booth, J. 1993, ApJ, 407, 699

Pilachowski, C. A., Sneden, C., \& Kraft, R. P. 1996a, AJ, 111, 1689

Pilachowski, C. A., Sneden, C., Kraft, R. P., Harmer, D., \& Willmarth, D. 2000, AJ, 119, 2895

Pilachowski, C. A., Sneden, C., Kraft, R. P., \& Langer, G. E. 1996b, AJ, 112,545

Preston, G. W. 1997, AJ, 113, 1860

Pryor, C. P., Latham, D. W., \& Hazen, M. L. 1988, AJ, 96, 123

Rappaport, S., Podsiadlowski, P., Joss, P. C., Di Stefano, R., \& Han, Z. 1995, MNRAS, 273, 731

Recio-Blanco, A., Piotto, G., Aparicio, A., \& Renzini, A. 2002, ApJ, 572, L71
Reddy, B. E., Lambert, D. L., Hrivnak, B. J., \& Bakker, E. J. 2002, AJ, 123, 1993

Reid, I. N. 2002, PASP, 114, 306

Renzini, A., \& Fusi Pecci, F. 1988, ARA\&A, 26, 199

Rufener, F., \& Bartholdi, P. 1982, A\&AS, 48, 503

Ryan, S. G., Norris, J. E., \& Bessell, M. S. 1991, AJ, 102, 303

Schwarzschild, M. 1975, ApJ, 195, 137

Shetrone, M. D. 1996, AJ, 112, 1517

Shetrone, M. D., Smith, G. H., Briley, M. M., Sandquist, E., \& Kraft, R. P. 1999, PASP, 111, 1115

Siess, L., \& Livio, M. 1999, MNRAS, 308, 1133 (SL99)

Sills, A., \& Pinsonneault, M. H. 2000, ApJ, 540, 489

Smith, G. H. 1998, PASP, 110, 1119

Smith, G. H., \& Dupree, A. K. 1988, AJ, 95, 1547

Smith, V. V., Shetrone, M. D., \& Keane, M. J. 1999, ApJ, 516, L73

Sneden, C., Kraft, R. P., Langer, G. E., Prosser, C. F., \& Shetrone, M. D. 1994, AJ, 107, 1773

Sneden, C., Kraft, R. P., Prosser, C. F., \& Langer, G. E. 1991, AJ, 102, 2001

. 1992, AJ, 104, 2121

Sneden, C., Kraft, R. P., Shetrone, M. D., Smith, G. H., Langer, G. E., \& Prosser, C. F. 1997, AJ, 114, 1964

Soker, N. 1992, ApJ, 386, 190 1996, ApJ, 460, L53

1998, AJ, 116, 1308

2001, MNRAS, 324, 699

Stone, R. P. S. 1983, PASP, 95, 27

Straniero, O., \& Chieffi, A. 1991, ApJS, 76, 525

Thomas, H.-C. 1967, Z. Astrophys., 67, 420

Wallerstein, G., \& Sneden, C. 1982, ApJ, 255, 577

Webbink, R. F. 1976, ApJ, 209, 829

Verbunt, F., \& Phinney, E. S. 1995, A\&A, 296, 709

Vogt, S. S., \& Penrod, G. D. 1983, PASP, 95,565

Zahn, J.-P. 1977, A\&A, 57, 383 (erratum 67, 162 [1978])

. 1989, A\&A, 220, 112

Zucker, S., \& Mazeh, T. 2001, ApJ, 562, 1038 